\title{
The signature of a fibre bundle is multiplicative mod 4
}

\author{
IAN HAMBLETON \\ ANDREW KORZENIEWSKI \\ ANDREW RANICKI
}

\begin{abstract}
We express the signature modulo 4 of a closed, oriented, $4 k$-dimensional $P L$ manifold as a linear combination of its Euler characteristic and the new absolute torsion invariant defined by Korzeniewski [11]. Let $F \rightarrow E \rightarrow B$ be a $P L$ fibre bundle, where $F, E$ and $B$ are closed, connected, and compatibly oriented $P L$ manifolds. We give a formula for the absolute torsion of the total space $E$ in terms of the absolute torsion of the base and fibre, and then combine these two results to prove that the signature of $E$ is congruent modulo 4 to the product of the signatures of $F$ and $B$.
\end{abstract}

$55 \mathrm{R} 25$

\section{Introduction}

The signature $\operatorname{sign}(M) \in \mathbb{Z}$ of a closed, oriented $n$-manifold $M^{n}$ is the index of its cup product form when $n \equiv 0 \bmod 4$, and zero otherwise. For an orientable differentiable fibre bundle, Chern, Hirzebruch and Serre [4] proved that $\operatorname{sign}(E)=\operatorname{sign}(F) \cdot \operatorname{sign}(B)$ provided that the fundamental group $\pi_{1}(B)$ acts trivially on the real cohomology $H^{*}(F ; \mathbb{R})$ of the fibre. In general, the signature is not multiplicative for differentiable fibre bundles: the first examples were constructed by Kodaira [10], Atiyah [2] and Hirzebruch [7]. These examples occur in the lowest possible dimension where $\operatorname{dim} B=$ $\operatorname{dim} F=2$. The total space $E$ of the bundle in these examples has dimension 4 and non-zero signature, but $\operatorname{sign}(B)=\operatorname{sign}(F)=0$ by convention. Surface bundles over surfaces were studied in detail by W Meyer [16] and H Endo [5]. For such bundles, the signature of the total space was shown to be divisible by 4 .

The non-multiplicative behaviour of the signature in fibre bundles is explained by the characteristic class formula for $\operatorname{sign}(E)$ due to Atiyah [2], which contains a contribution from the action of $\pi_{1}(B)$ on the cohomology of the fibre. On the other hand, W Neumann [19] showed that the signature is multiplicative in many cases where the structural group of the bundle is discrete, and gave a Witt group interpretation of the obstruction to multiplicativity under this assumption. 
Stephan Klaus and Peter Teichner [9] have conjectured that the signature is multiplicative modulo 8 , provided that $\pi_{1}(B)$ acts trivially on the mod 2 cohomology $H^{*}(F ; \mathbb{Z} / 2)$ of the fibre. This suggests studying the conditions under which the signature is multiplicative modulo other powers of 2 . The first case is easy since $\operatorname{sign}(E) \equiv \chi(E) \bmod 2$, and the Euler characteristic $\chi(E)=\chi(F) \cdot \chi(B)$ is multiplicative. The following is our main result.

Theorem A Let $F \stackrel{q}{\rightarrow} E \stackrel{p}{\rightarrow} B$ be a $P L$ fibre bundle of closed, connected, compatibly oriented PL manifolds. Then

$$
\operatorname{sign}(E) \equiv \operatorname{sign}(F) \cdot \operatorname{sign}(B) \bmod 4 .
$$

We will use the new absolute torsion invariant $\tau^{\mathrm{NEW}}(E) \in K_{1}\left(\mathbb{Z}\left[\pi_{1}(E)\right]\right)$ defined and investigated by Korzeniewski [11]. The new invariant is defined more generally, and has better properties than the "round torsion" invariant introduced by Ranicki [24]. We show in Theorem 3.3 that the value of $\operatorname{sign}(E) \bmod 4$ can be expressed in terms of $\tau^{\mathrm{NEW}}(E)$ and $\chi(E)$. Our main result follows from this fact, and a general formula for $\tau^{\mathrm{NEW}}(E)$ in terms of $\tau^{\mathrm{NEW}}(F)$ and $\tau^{\mathrm{NEW}}(B)$.

Theorem B Let $F \stackrel{q}{\rightarrow} E \stackrel{p}{\rightarrow} B$ be a PL fibre bundle of closed, connected, compatibly oriented $P L$ manifolds. Then, if $n=\operatorname{dim} E$,

$$
\tau^{\mathrm{NEW}}(E)=p^{!}\left(\tau^{\mathrm{NEW}}(B)\right)+\chi(B) q_{*}\left(\tau^{\mathrm{NEW}}(F)\right) \in \hat{H}^{n}\left(\mathbb{Z} / 2 ; K_{1}\left(\mathbb{Z} \pi_{1}(E)\right)\right) .
$$

In this statement the maps $p^{!}$and $q_{*}$ are the transfer and push-forward maps associated to the fibre bundle (see Lück [12;13] and Lück-Ranicki [14] for the surgery transfer).

In Section 1 we describe some of the results of [11], including the definition and main properties of the absolute torsion invariant. In Section 2 we use the new invariant to correct the definition of the round simple symmetric $L$-groups of Hambleton-RanickiTaylor [6], so they fit in the long exact sequence

$$
\cdots \rightarrow L_{r s}^{n}(R) \longrightarrow L_{r h}^{n}(R) \stackrel{\tau^{\mathrm{NEW}}}{\longrightarrow} \widehat{H}^{n}\left(\mathbb{Z} / 2 ; K_{1}(R)\right) \longrightarrow L_{r s}^{n-1}(R) \rightarrow \cdots
$$

Section 3 establishes the relationship between the signature mod 4 and the new torsion invariant of a $4 k$-dimensional Poincaré space (see Theorem 3.3). Section 4 and Section 5 study the set $\mathcal{S}_{\bullet}(X)$ of pointed torsion structures (PTS) on manifolds. In Theorem 5.2 we give a formula expressing the absolute torsion invariant for a closed manifold $X$ in terms of a pairing

$$
\Phi: \mathcal{S}_{\bullet}(X) \times \mathcal{S}_{\bullet}(X) \rightarrow K_{1}\left(\mathbb{Z}\left[\pi_{1}\left(X, x_{0}\right)\right]\right) .
$$


After this preparation, we turn to the geometric applications of absolute torsion for fibre bundles.In Section 6 we define the fibre transport and transfer map $p^{!}$associated to a fibre bundle, and establish some useful properties. Section 7 and Section 8 deal with pointed torsion structures on fibre bundles, and the $\Phi$-pairing for the total space $E$. Section 9 uses the results of Section 8 to prove Theorem B and then Section 10 uses Theorem B to prove Theorem A. Section 11 is a brief discussion of fibrations of Poincaré spaces.

The last part of the paper (Section 12) contains the algebra needed to describe the absolute torsion of signed filtered chain complexes, such as the chain complexes of the total spaces of fibre bundles. This may be of independent interest, and this part can be read independently of the rest of the paper. We give a purely algebraic treatment of the absolute torsion of filtered chain complexes: the main result is the Invariance Theorem 12.14 identifying the torsion of a contractible filtered chain complex with the torsion of the contractible chain complex (in the derived category) of filtration quotients.

We are indebted to Stephan Klaus and Peter Teichner for informing us about their results, and pointing out this related question to the first author in discussions at Oberwolfach in July 2001.

\section{Thanks}

Research partially supported by NSERC Research Grant A 4000, the British Association Travel Fund, and the Edinburgh Mathematical Society. The authors also wish to thank the Max Planck Institut für Mathematik, Bonn, for its hospitality and support.

\section{Signed $K$-theory}

In this section we recall the algebraic $K$-groups $K_{0}(R), K_{1}(R)$ of a ring $R$, and the algebraic theory of torsion of Ranicki [24; 25; 26] and Korzeniewski [11]. The absolute torsion invariant $\tau^{\mathrm{NEW}}(f) \in K_{1}(R)$ of [11] is based on the definition of "round torsion" given in $[26,7.20($ ii) $]$, but the sign correction terms are different.

The class group $K_{0}(\mathbb{A})$ of an additive category $\mathbb{A}$ is the abelian group with one generator $[M]$ for each object $M$ in $\mathbb{A}$, and relations

(i) $[M]=\left[M^{\prime}\right]$ if $M$ is isomorphic to $M^{\prime}$ and

(ii) $[M \oplus N]=[M]+[N]$ for objects $M, N$ in $\mathbb{A}$.

The isomorphism torsion group $K_{1}^{\text {iso }}(\mathbb{A})$ is the abelian group with one generator $\tau^{\text {iso }}(f)$ for each isomorphism $f: M \rightarrow N$ in $\mathbb{A}$, and relations 
(i) $\tau^{\text {iso }}(g f)=\tau^{\text {iso }}(f)+\tau^{\text {iso }}(g)$ for isomorphisms $f: M \rightarrow N, g: N \rightarrow P$ and

(ii) $\tau^{\text {iso }}\left(\left(\begin{array}{cc}f & e \\ 0 & f^{\prime}\end{array}\right)\right)=\tau^{\text {iso }}(f)+\tau^{\text {iso }}\left(f^{\prime}\right)$ for isomorphisms $f: M \rightarrow N, f^{\prime}: M^{\prime} \rightarrow N^{\prime}$ and any morphism $e: M^{\prime} \rightarrow N$.

The class group $K_{0}(R)$ and the isomorphism torsion group $K_{1}^{\text {iso }}(R)$ of a ring $R$ are the class and torsion groups of the additive category $\mathbb{A}(R)$ of based finitely generated (f.g.) free $R$-modules. To obtain values in $K_{1}(R)$, we compose with the split surjection $K_{1}^{\text {iso }}(R) \rightarrow K_{1}(R)$ given by [24, Proposition 5.1], and let $\tau^{\text {iso }}(f) \mapsto \tau(f) \in K_{1}(R)$. In particular, there are defined isomorphisms

$$
\begin{aligned}
& K_{0}(\mathbb{Z}) \stackrel{\cong}{\longrightarrow} \mathbb{Z} ;[M] \mapsto \operatorname{rank}_{\mathbb{Z}} M, \\
& K_{1}(\mathbb{Z}) \stackrel{\cong}{\longrightarrow} \mathbb{Z} / 2 ;[M, f] \mapsto \tau(\operatorname{det}(f)) .
\end{aligned}
$$

The reduced class group $\widetilde{K}_{0}(R)$ and the reduced torsion group $\widetilde{K}_{1}(R)$ are defined by

$$
\tilde{K}_{i}(R)=\operatorname{coker}\left(K_{i}(\mathbb{Z}) \rightarrow K_{i}(R)\right) \quad(i=0,1) .
$$

We shall assume that $R$ is such that the rank of f.g. free $R$-modules is well-defined, so that $K_{0}(R)=K_{0}(\mathbb{Z}) \oplus \widetilde{K}_{0}(R)$.

We shall consider only finite chain complexes over $R$, meaning finite dimensional, positive chain complexes of f.g. free $R$-modules. The Euler characteristic of a finite $R$-module chain complex $C$ is the chain homotopy invariant

$$
\chi(C)=\sum_{r=0}^{\infty}(-1)^{r} \operatorname{rank}_{R} C_{r} \in \mathbb{Z} .
$$

Our assumptions on $C$ mean that $C_{r}=0$ if $r<0$ or $r>N$ for some integer $N$, so this sum is defined. A finite chain complex $C$ is round if $\chi(C)=0 \in \mathbb{Z}$.

The torsion $\tau(C) \in K_{1}(R)$ of a contractible finite based $R$-module chain complex

$$
C: 0 \rightarrow \cdots \rightarrow C_{r+1} \stackrel{d}{\longrightarrow} C_{r} \stackrel{d}{\longrightarrow} C_{r-1} \rightarrow \cdots \rightarrow 0
$$

was originally defined by Whitehead [29] to be

$$
\tau(C)=\tau(d+\Gamma) \in K_{1}(R),
$$

with the isomorphism

$$
d+\Gamma: C_{\mathrm{odd}}=\sum_{r \text { odd }} C_{r} \longrightarrow C_{\mathrm{even}}=\sum_{r \text { even }} C_{r}
$$


defined using any contraction $\Gamma: 1 \simeq 0$ : $C \rightarrow C$. The value of $\tau(C)$ is independent of the choice of $\Gamma$.

The torsion of a chain equivalence $f: C \rightarrow D$ of finite based $R$-module chain complexes can be defined by

$$
\tau(f)=\tau(\mathcal{C}(f)) \in K_{1}(R)
$$

with $\mathcal{C}(f)$ the algebraic mapping cone

$$
d_{\mathcal{C}(f)}=\left(\begin{array}{cc}
d_{D} & (-)^{r-1} f \\
0 & d_{C}
\end{array}\right): \mathcal{C}(f)_{r}=D_{r} \oplus C_{r-1} \rightarrow \mathcal{C}(f)_{r-1}=D_{r-1} \oplus C_{r-2} .
$$

However, as noted in [24], this definition of torsion only has good sum and composition properties modulo the sign subgroup

$$
\operatorname{im}\left(K_{1}(\mathbb{Z}) \rightarrow K_{1}(R)\right)=\{\tau( \pm 1)\} \subseteq K_{1}(R) .
$$

The new absolute torsion uses the notion of a signed chain complex [11, Definition 5]. This is a pair $\left(C, \eta_{C}\right)$, where $C$ is as above and $\eta_{C} \in \operatorname{Im}\left(K_{1}(\mathbb{Z}) \rightarrow K_{1}(R)\right)$. The image of $K_{1}(\mathbb{Z})=\mathbb{Z} / 2$ in $K_{1}(R)$ is given by $a \mapsto a \cdot \tau(-1)$, but the symbol

$$
\tau(-1):=\tau(-1: R \rightarrow R) \in K_{1}(R)
$$

will usually be suppressed to simplify the notation.

If $\left(C, \eta_{C}\right)$ is a signed chain complex with $C$ contractible, then its absolute torsion is defined as

$$
\tau^{\mathrm{NEW}}\left(C, \eta_{C}\right)=\tau(C)+\eta_{C} .
$$

If $\left(C, \eta_{C}\right)$ is a signed complex, then the suspension $S C$ of $C$, where $(S C)_{r}=C_{r-1}$, has the sign $\eta_{S C}=-\eta_{C}$. The sum $\left(C \oplus D, \eta_{C \oplus D}\right)$ of two signed complexes involves some more sign terms from $K_{1}(\mathbb{Z})=\mathbb{Z} / 2$. Let

$$
\epsilon(M, N):=\operatorname{rank}_{R}(M) \cdot \operatorname{rank}_{R}(N) \bmod 2
$$

for any free $R$-modules $M$ and $N$, and define [24, page 213]:

$$
\beta(C, D)=\sum_{i>j}\left(\epsilon\left(C_{2 i}, D_{2 j}\right)-\epsilon\left(C_{2 i+1}, D_{2 j+1}\right)\right) \in \operatorname{Im}\left(K_{1}(\mathbb{Z}) \rightarrow K_{1}(R)\right) .
$$

This is just the difference of the torsions of the permutation isomorphisms $(C \oplus$ $D)_{\text {even }} \rightarrow C_{\text {even }} \oplus D_{\text {even }}$ and $(C \oplus D)_{\text {odd }} \rightarrow C_{\text {odd }} \oplus D_{\text {odd }}$. We define

$$
\eta_{C \oplus D}=\eta_{C}+\eta_{D}-\beta(C, D)+\operatorname{rank}\left(C_{\text {odd }}\right) \cdot \chi(D) .
$$

From now on, we will usually denote a signed chain complex $\left(C, \eta_{C}\right)$ just by $C$, even though all the formulas will involve these signs. It turns out that the absolute torsion of 
a Poincaré complex $C$ is independent of the choice of sign $\eta_{C}$, and this justifies our abbreviated notation (see [11, Proposition 26.6]).

The absolute torsion of a chain equivalence $f: C \rightarrow D$ of finite based signed complexes is defined to be

$$
\tau^{\mathrm{NEW}}(f)=\tau^{\mathrm{NEW}}(\mathcal{C}(f)) \in K_{1}(R),
$$

with $\eta_{\mathcal{C}(f)}=\eta_{D \oplus S C}$. The extra sign terms needed (in comparison with the invariant of [24]) can be seen from the formula

$$
\tau^{\mathrm{NEW}}(f)=\tau(C(f))-\beta(D, S C)+\operatorname{rank}_{R}\left(D_{\text {odd }}\right) \cdot \chi(S C)+\eta_{D}-\eta_{C} \in K_{1}(R)
$$

given in [11, Lemma 12].

For chain equivalences of signed complexes, the absolute torsion invariant is a chain homotopy invariant, which is additive under compositions and direct sums (by an appropriate modification of the proof of [24, Propositions 4.2, 4.4 and 4.5]).

Proposition 1.1 (Korzeniewski [11, Proposition 13]) If $f: C \rightarrow D$ and $g: D \rightarrow E$ are chain equivalences of finite based signed chain complexes, then

$$
\tau^{\mathrm{NEW}}(g \circ f)=\tau^{\mathrm{NEW}}(f)+\tau^{\mathrm{NEW}}(g) \in K_{1}(R) .
$$

If $f: C \rightarrow D$ and $f^{\prime}: C^{\prime} \rightarrow D^{\prime}$ are chain equivalences of finite based signed chain complexes, then

$$
\tau^{\mathrm{NEW}}\left(f \oplus f^{\prime}\right)=\tau^{\mathrm{NEW}}(f)+\tau^{\mathrm{NEW}}\left(f^{\prime}\right) \in K_{1}(R) .
$$

The formula for the absolute torsion is a modification of the definition in [24, pages $223,226]$, which gave an invariant with values in $K_{1}^{\text {iso }}(\mathbb{A})$ for chain complexes over an additive category $\mathbb{A}$. In that setting, the signs $\eta_{C}$ are in the image of the skew-symmetric pairing

$$
\epsilon: K_{0}(\mathbb{A}) \otimes K_{0}(\mathbb{A}) \rightarrow K_{1}^{\text {iso }}(\mathbb{A})
$$

defined in [24, Proposition 2.2]: for any two objects $M, N$ of $\mathbb{A}$,

$$
\epsilon([M],[N])=\tau^{\text {iso }}(M \oplus N \rightarrow N \oplus M) \in K_{1}^{\text {iso }}(\mathbb{A})
$$

is the torsion of the interchange map. See [11, Lemma 7] for the properties of this pairing.

This extra generality will be useful in Section 12 where we discuss the absolute torsion of filtered chain complexes. A finite chain complex over $\mathbb{A}$ is an object in the derived category $\mathbb{D}(\mathbb{A})$, whose morphisms are the chain homotopy classes of chain maps. 
Definition 1.2 The signed derived category $\operatorname{SD}(A)$ of an additive category $\mathbb{A}$ is the additive category with objects signed complexes $\left(C, \eta_{C}\right)$ in $\mathbb{A}$, and morphisms the chain homotopy classes of chain maps. An isomorphism in $\mathbb{S} \mathbb{D}(\mathbb{A})$ is a chain homotopy class of chain equivalences.

If $f: C \rightarrow D$ is a morphism in $\operatorname{SDD}(\mathbb{A})$, then the mapping cone $\mathcal{C}(f)$ is an object in $\mathbb{S D}(\mathbb{A})$ under our sign conventions. The absolute torsion gives an invariant in $\tau^{\mathrm{NEW}}(f) \in K_{1}^{\text {iso }}(\mathbb{S} \mathbb{D}(\mathbb{A}))$ for an isomorphism $f: C \rightarrow D$ in $\mathbb{S} \mathbb{D}(\mathbb{A})$. We refer to [11] for the definition and properties of the absolute torsion in this setting, but we note that the map

$$
i_{*}: K_{1}^{\text {iso }}(\mathbb{S} \mathbb{D}(\mathbb{A})) \rightarrow K_{1}^{\text {iso }}(\mathbb{A})
$$

defined by $i_{*} \tau^{\text {iso }}(f)=\tau^{\mathrm{NEW}}(f)$ is a split surjection. The map $i_{*}$ has the naturality property

$$
i_{*} \epsilon(C, D)=\epsilon(\chi(C), \chi(D)) \in K_{1}^{\text {iso }}(\mathbb{A})
$$

for any objects $\left(C, \eta_{C}\right),\left(D, \eta_{D}\right)$ in $\mathbb{S} \mathbb{D}(\mathbb{A})$. We will also use the relation

$$
i_{*} \epsilon(C, S D)=-i_{*} \epsilon(C, D) \in K_{1}^{\text {iso }}(\mathbb{A})
$$

and the formula

$$
\eta_{C(f)}=\eta_{D \oplus S C}=\eta_{D}-\eta_{C}-\beta(D, S C)+\epsilon\left(D_{o d d}, \chi(S C)\right) \in K_{1}^{\text {iso }}(\mathbb{S D}(\mathbb{A}))
$$

for the mapping cone sign in our sign calculations (see Section 12).

\section{Round $L$-theory}

In this section we recall the round $L$-theory of Hambleton, Ranicki and Taylor [6]. Note that the round torsion is not a round cobordism invariant, contrary to the assertion in [26, page 190], but the new absolute torsion of [11] does have this important property. Moreover, all the results claimed in [26, 7.22(ii)] and [6, page 135] do hold after replacing the round torsion with the new absolute torsion invariant.

A finite, oriented Poincaré duality space $X$ of dimension $n$, with universal covering $\tilde{X}$, has an associated symmetric chain complex $(C, \varphi)$, as defined in [22]. Here $C:=C(\tilde{X})$ is a $n$-dimensional finite chain complex over $R=\mathbb{Z}\left[\pi_{1}(X)\right]$ and $\varphi_{0}: C^{n-*} \rightarrow C_{*}$ is a chain equivalence. The definition of this symmetric structure $\varphi$ uses the standard involution $g \mapsto g^{-1}$ on the group ring. More generally, for any ring $R$ with involution $\alpha$, and unit $\varepsilon= \pm 1$, Ranicki [22] defines $\varepsilon$-symmetric structures on algebraic chain complexes and the notion of a symmetric (algebraic) Poincaré complex. The cobordism group of $n$-dimensional symmetric Poincaré is denoted $L^{n}(R, \varepsilon)$ [22, Section 3]. The 
additive inverse is defined to be $-(C, \varphi):=(C,-\varphi)$. In the rest of the paper we will assume that $\varepsilon=+1$, and denote these groups just by $L^{n}(R)$.

If we restrict to round complexes in both objects and bordisms and $n>0$, we get the round symmetric $L$-groups $L_{r}^{n}(R)$. The groups $L_{r}^{0}(R)$ is the Witt group of formal differences $(M, \psi)-\left(M^{\prime}, \psi^{\prime}\right)$ of non-singular symmetric forms over $(R, \alpha)$ with $\operatorname{rank}_{R} M=\operatorname{rank}_{R} M^{\prime}$ [6, Proposition 2.1]. We can compare the round $L$-groups to the ordinary ones by a long exact sequence. The anti-automorphism on $R$ induces a $\mathbb{Z} / 2$-action on $K_{0}(R)$ which is the identity on the subgroup $K_{0}(\mathbb{Z})=\mathbb{Z}$.

Proposition 2.1 (Hambleton-Ranicki-Taylor [6, Proposition 3.2]) For any ring with involution $(R, \alpha)$, there is a long exact sequence

$$
\cdots \rightarrow \hat{H}^{n+1}\left(\mathbb{Z} / 2 ; K_{0}(\mathbb{Z})\right) \rightarrow L_{r}^{n}(R) \rightarrow L^{n}(R) \rightarrow \hat{H}^{n}\left(\mathbb{Z} / 2 ; K_{0}(\mathbb{Z})\right) \rightarrow \cdots
$$

where the map $L^{n}(R) \rightarrow \widehat{H}^{n}\left(\mathbb{Z} / 2 ; K_{0}(\mathbb{Z})\right)$ is defined by $(C, \varphi) \mapsto \chi(C)$.

We will also need to compare these $L$-groups to the cobordism groups $L_{e v}^{n}(R)$ of $n$-dimensional symmetric Poincaré complexes $(C, \varphi)$ with even Euler characteristic $\chi(C) \equiv 0 \bmod 2$ for objects and bordisms:

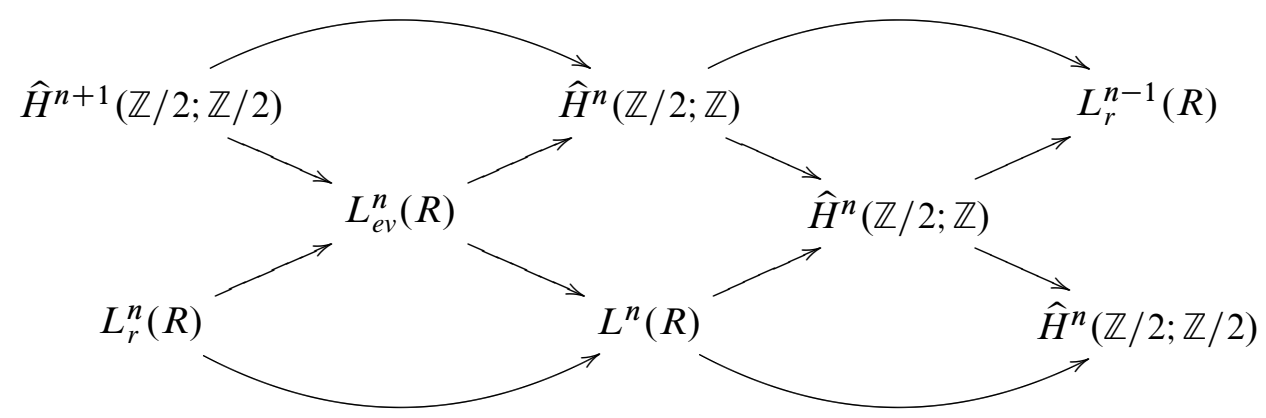

The map $L_{e v}^{n}(R) \rightarrow \widehat{H}^{n}(\mathbb{Z} / 2 ; \mathbb{Z})$ is given by $(C, \varphi) \mapsto \chi(C) / 2$.

In order to define the torsion of a symmetric Poincare chain complex over a ring with involution $(R, \alpha)$, we need to work with signed chain complexes, in which each free chain module $C_{r}$ has a preferred basis, along with a single choice of sign $\eta_{C}$. We denote by $L_{r h}^{n}(R)$ the corresponding cobordism group of round, $n$-dimensional, finite, signed symmetric Poincaré chain complexes of finitely generated based free $R$-modules. By forgetting the preferred bases and signs we can identify $L_{r h}^{n}(R)=L_{r}^{n}(R)$. Similarly, we can identify the based and unbased versions of the even $L$-groups $L_{e v h}^{n}(R)=L_{e v}^{n}(R)$.

Now let $(C, \varphi)$ denote an $n$-dimensional signed symmetric Poincaré chain complex, of finitely generated based free $R$-modules, over a ring with involution $(R, \alpha)$. The 
absolute torsion

$$
\tau^{\mathrm{NEW}}(C, \varphi):=\tau^{\mathrm{NEW}}\left(\varphi_{0}: C^{n-*} \rightarrow C_{*}\right) \in K_{1}(R)
$$

is now defined, as the absolute torsion of the chain equivalence $\varphi_{0}$ with respect to the given bases on $C_{*}$ and the dual bases on $C^{n-*}$. Recall that the differential for $C^{n-*}$ has the sign convention

$$
d_{C^{n-*}}=(-1)^{r} d_{C}^{*}: C^{n-r} \rightarrow C^{n-r+1} .
$$

The dual signed chain complex $\left(C^{n-*}, \eta_{C^{n-*}}\right)$ is given the sign

$$
\eta_{C^{n-*}}=\eta_{C}+\beta(C, C)+\alpha_{n}(C) \in \operatorname{Im}\left(K_{1}(\mathbb{Z}) \rightarrow K_{1}(R)\right)
$$

where

$$
\alpha_{n}(C)=\sum_{r \equiv n+2, n+3(\bmod 4)} \operatorname{rank}_{R}\left(C^{r}\right) \in \operatorname{Im}\left(K_{1}(\mathbb{Z}) \rightarrow K_{1}(R)\right) .
$$

With this convention, the absolute torsion $\tau^{\mathrm{NEW}}(C, \varphi)$ is independent of the initial choice of sign $\eta_{C}$ (see [11, Proposition 26]). We say that $(C, \varphi)$ is simple if $\tau^{\mathrm{NEW}}(C, \varphi)=0$.

Lemma 2.2 [11, Lemma 10] If $\varphi_{0}: C^{n-*} \rightarrow C$ is an isomorphism, then

$$
\tau^{\mathrm{NEW}}(C, \varphi)=\sum_{r=0}^{n}(-1)^{r} \tau\left(\varphi_{0}: C^{n-r} \rightarrow C_{r}\right)+\beta(C, C)+\alpha_{n}(C) \in K_{1}(R) .
$$

Proof The absolute torsion of a chain isomorphism $f: C \rightarrow D$ of signed chain complexes is just

$$
\tau^{\mathrm{NEW}}(f)=\sum_{r=0}^{n}(-1)^{r} \tau\left(f_{r}: C_{r} \rightarrow D_{r}\right)-\eta_{C}+\eta_{D}
$$

according to [11, Definition 6]. The sign terms for $\varphi_{0}$ appear above.

The absolute torsion has the symmetry property [11, Propostion 26.2] (compare [26, 7.20(ii)])

$$
\tau^{\mathrm{NEW}}(C, \varphi)^{*}=(-1)^{n} \tau^{\mathrm{NEW}}(C, \varphi)+\frac{1}{2} n(n+1) \chi(C)
$$

where $*: K_{1}(R) \rightarrow K_{1}(R)$ denotes the involution on $K$-theory induced by " $\alpha-$ conjugate-transpose" of matrices. The sign term vanishes over rings $R$ for which skew-hermitian forms necessarily have even rank (such as an integral group ring $R=\mathbb{Z}[\pi])$. Also

$$
\tau^{\mathrm{NEW}}(C,-\varphi)=\tau^{\mathrm{NEW}}(C, \varphi)+\chi(C)
$$


(see [11, Proposition 26.5]). Notice that the extra sign terms in both formulas vanish for round or even symmetric complexes. Moreover, the absolute torsion is additive under direct sums of symmetric $n$-complexes. It follows that $\tau^{\mathrm{NEW}}(C, \varphi)$ defines an additive map into $\hat{H}^{n}\left(\mathbb{Z} / 2 ; K_{1}(R)\right)$ for round or even symmetric complexes.

For our applications, we will need the cobordism groups of simple round or even Poincaré complexes, and their relation to the $L$-groups already mentioned. However, to give a well-defined homomorphism

$$
L_{e v}^{n}(R) \rightarrow \hat{H}^{n}\left(\mathbb{Z} / 2 ; K_{1}(R)\right)
$$

we need the invariant associated to a null-bordant symmetric complex to be trivial.

Example 2.3 Let $R=\mathbb{Z}$ with trivial involution. A zero-dimensional symmetric Poincaré complex over $\mathbb{Z}$ is just a unimodular, symmetric bilinear form $(L, h)$ on a finitely-generated free abelian group. In this case,

$$
\tau^{\mathrm{NEW}}(L, h)=\tau(\operatorname{det} h) \in \mathbb{Z} / 2
$$

by Lemma 2.2. To compare this invariant to the determinant, we map $\mathbb{Z} / 2 \rightarrow \mathbb{Z} / 4$ by $a \mapsto 2 a \bmod 4$. Then $2 \tau(\operatorname{det} h) \equiv \operatorname{det} h-1 \bmod 4$ and

$$
2 \tau^{\mathrm{NEW}}(L, h) \equiv \operatorname{det} h-1 \bmod 4 .
$$

Note that for any form $(L, h)$ of even rank, the absolute torsion is just $\tau(\operatorname{det} h)$. In particular, the absolute torsion of the hyperbolic plane $\mathbb{W}$ is non-zero, so $\mathbb{U}$ represents a non-trivial element of $L_{e v}^{0}(\mathbb{Z})$.

Lemma 2.4 [11, Proposition 26.4] If $(C, \varphi)$ is homotopy equivalent to the boundary of an $(n+1)$-dimensional symmetric signed complex $(D, \Phi)$, then

$\tau^{\mathrm{NEW}}(C, \varphi)=(-1)^{n+1} \tau^{\mathrm{NEW}}(C \rightarrow \partial D)^{*}-\tau^{\mathrm{NEW}}(C \rightarrow \partial D)+\frac{1}{2}(n+1)(n+2) \chi(D)$.

Once again, the extra sign term vanishes for round or even complexes.

Corollary 2.5 The absolute torsion $\tau^{\mathrm{NEW}}(C, \varphi)$ induces a well-defined homomorphism $L_{e v}^{n}(R) \rightarrow \hat{H}^{n}\left(\mathbb{Z} / 2 ; K_{1}(R)\right)$.

The cobordism group of simple round (or even) symmetric Poincaré complexes is denoted $L_{r s}^{n}(R)$ or $L_{e v s}^{n}(R)$, following [6, page 135]. 
Proposition 2.6 There is a commutative braid of exact sequences

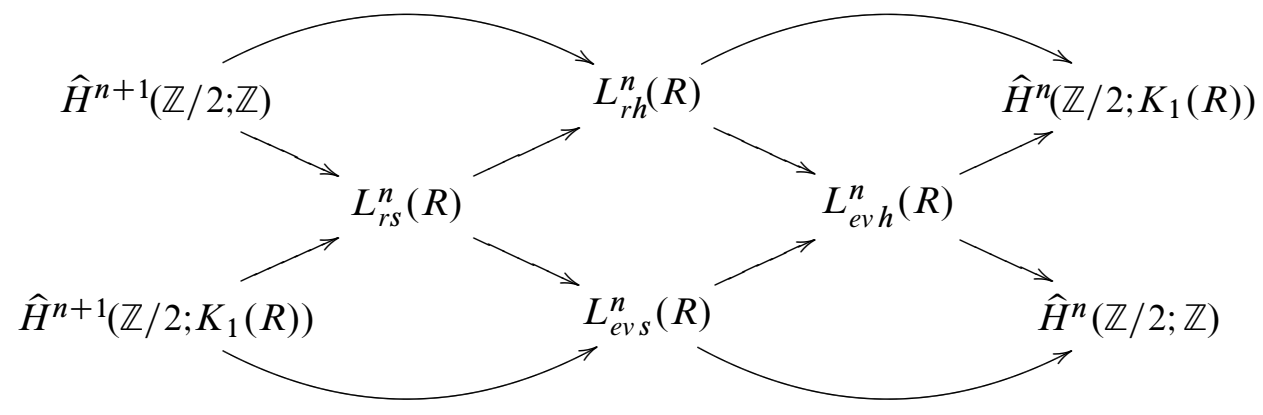

where the maps $L_{r h}^{n}(R) \rightarrow \widehat{H}^{n}\left(\mathbb{Z} / 2 ; K_{1}(R)\right)$ and $L_{\text {ev } h}^{n}(R) \rightarrow \widehat{H}^{n}\left(\mathbb{Z} / 2 ; K_{1}(R)\right)$ are induced by the absolute torsion $(C, \varphi) \mapsto \tau^{\mathrm{NEW}}\left(\varphi_{0}\right) \in K_{1}(R)$. The maps to $\hat{H}^{n}(\mathbb{Z} / 2 ; \mathbb{Z})$ are given by $(C, \varphi) \mapsto \chi(C) / 2$.

Lemma 2.7 Let $(R, \alpha)$ be a ring with involution, and $(C, \varphi)$ be an even based symmetric Poincaré complex over $R$, with $\chi(C) \equiv 0 \bmod 4$ and $\left\{\tau^{\mathrm{NEW}}(C, \varphi)\right\}=$ $0 \in \widehat{H}^{n}\left(\mathbb{Z} / 2 ; K_{1}(R)\right)$. Then $(C, \varphi)$ is even Poincaré bordant to a round simple based symmetric Poincaré complex $\left(C^{\prime}, \varphi^{\prime}\right)$.

Proof This follows from the comparison braid given in Proposition 2.6 relating $L_{r h}^{n}(R)$ and $L_{e v h}^{n}(R)$.

Example 2.8 For the special case $R=\mathbb{Z}$ and $n=0$, we can substitute the calculation $L_{r h}^{0}(\mathbb{Z})=2 \mathbb{Z}$ from $[6,4.2]$ :

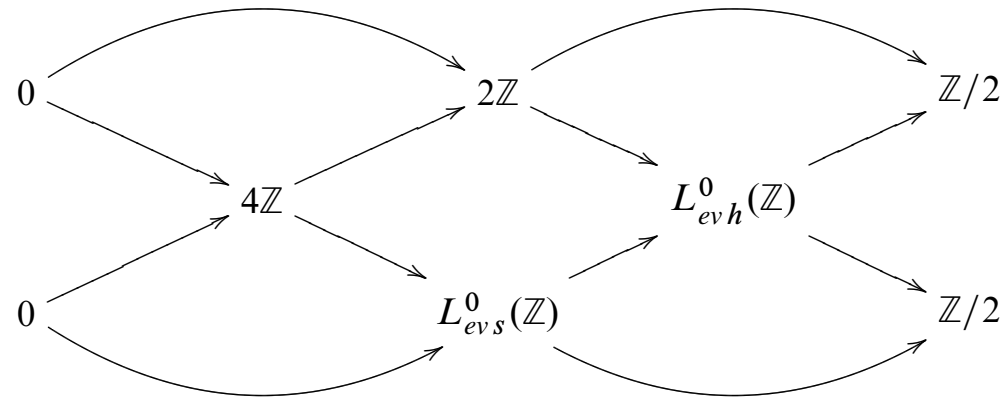

We obtain $L_{r s}^{0}(\mathbb{Z})=4 \mathbb{Z}$. Since the hyperbolic plane $\mathbb{H}$ represents a non-zero element of $L_{e v h}^{0}(\mathbb{Z})$ with $\chi \equiv 2 \bmod 4$ and $\operatorname{det}(\mathbb{Z})=-1$, we have $L_{\text {ev } h}^{0}(\mathbb{Z})=2 \mathbb{Z} \oplus \mathbb{Z} / 2$, generated by $\langle 1\rangle \perp\langle 1\rangle$ and $\mathbb{Z}$, and $L_{\text {ev } S}^{0}(\mathbb{Z})=2 \mathbb{Z}$ generated by $\langle 1\rangle \perp\langle 1\rangle$. A diagram chase gives $L_{\text {ev } h}^{2}(\mathbb{Z})=\mathbb{Z} / 2$ and the fact that $\left\{\tau^{\mathrm{NEW}}(C, \varphi)\right\}=0 \in \hat{H}^{0}\left(\mathbb{Z} / 2 ; K_{1}(\mathbb{Z})\right)$ if $\operatorname{dim} C \equiv 2 \bmod 4$. 
We will need the following calculation in a later section.

Lemma 2.9 Let $C$ be a symmetric Poincaré complex over $R=\mathbb{Z}[\mathbb{Z} / 2]$. The absolute torsion $\left\{\tau^{\mathrm{NEW}}(C, \varphi)\right\}=0 \in \hat{H}^{0}\left(\mathbb{Z} / 2 ; K_{1}(\mathbb{Z}[\mathbb{Z} / 2])\right)$ if $\operatorname{dim} C \equiv 2 \bmod 4$.

Proof Since $\operatorname{dim} C \equiv 2 \bmod 4, C$ is an even symmetric Poincaré complex, so $\tau^{\mathrm{NEW}}(C, \varphi)$ defines an element of $\hat{H}^{0}\left(\mathbb{Z} / 2 ; K_{1}(R)\right)$. Let $\mathbb{Z} / 2=\langle T\rangle$ denote a generator of the group of order two, and we have an inclusion $\mathbb{Z}[\mathbb{Z} / 2] \subset \mathbb{Z} \oplus \mathbb{Z}$ of rings given by $T \mapsto \pm 1$. The induced map

$$
\hat{H}^{0}\left(\mathbb{Z} / 2 ; K_{1}(\mathbb{Z}[\mathbb{Z} / 2])\right) \rightarrow \hat{H}^{0}\left(\mathbb{Z} / 2 ; K_{1}(\mathbb{Z} \oplus \mathbb{Z})\right)
$$

is injective, and the absolute torsion map $L_{\text {ev } h}^{4 k+2}(\mathbb{Z}[\mathbb{Z} / 2]) \rightarrow \hat{H}^{0}\left(\mathbb{Z} / 2 ; K_{1}(\mathbb{Z}[\mathbb{Z} / 2])\right)$ composed with this injection factors through $L_{e v h}^{2}(\mathbb{Z}) \rightarrow \widehat{H}^{0}\left(\mathbb{Z} / 2 ; K_{1}(\mathbb{Z})\right)$, which we have seen is the zero map.

\section{Absolute torsion and signatures}

Let $X$ be a finite, connected $C W$-complex, and fix a base point $x_{0} \in X$. We choose orientations and lifts to the universal covering $\tilde{X}$ for each cell in $X$, together with a sign $\eta_{X}$. The cellular chain complex $\left(C(\tilde{X}), \eta_{X}\right)$ is now a finitely-generated, signed, based chain complex over $R=\mathbb{Z}\left[\pi_{1}\left(X, x_{0}\right)\right]$. If $X$ is an oriented, finite, geometric Poincaré $n$-complex, then we define

$$
\tau^{\mathrm{NEW}}(X)=\tau^{\mathrm{NEW}}\left(C(\tilde{X}), \varphi_{0}\right) \in \hat{H}^{n}\left(\mathbb{Z} / 2 ; K_{1}\left(\mathbb{Z}\left[\pi_{1}\left(X, x_{0}\right)\right]\right)\right)
$$

where $\varphi_{0}$ is the duality map from the symmetric structure on $C(\tilde{X})$ defined by Ranicki [22, page 92]. The symmetric structure is a homotopy invariant of $X$.

Lemma 3.1 Let $X$ be an oriented, finite, Poincaré complex of dimension $n$. The absolute torsion $\tau^{\mathrm{NEW}}(X) \in \hat{H}^{n}\left(\mathbb{Z} / 2 ; K_{1}\left(\mathbb{Z}\left[\pi_{1}(X)\right]\right)\right)$ depends only on the homotopy type of $X$. In particular, the absolute torsion is independent of the choice of preferred base for $C(\tilde{X})$.

Proof The absolute torsion $\tau^{\mathrm{NEW}}(X)$ is independent of all the choices made by [11, Proposition 26].

The symmetric signature of $X$ is the element $\sigma^{*}(X) \in L^{n}\left(\mathbb{Z}\left[\pi_{1}\left(X, x_{0}\right)\right]\right)$ given by the bordism class of the symmetric structure $(C(\tilde{X}), \varphi)$. If $\chi(X) \equiv 0 \bmod 2$, then we get an even symmetric signature $\sigma_{e v}^{*}(X) \in L_{e v}^{n}\left(\mathbb{Z}\left[\pi_{1}(X)\right]\right)$. In this case, $\tau^{\mathrm{NEW}}(X)$ is 
a bordism invariant: it is the image of $\sigma_{e v}^{*}(X)$ under the homomorphism $L_{e v}^{n}(R) \rightarrow$ $\hat{H}^{n}\left(\mathbb{Z} / 2 ; K_{1}(R)\right)$ from Corollary 2.5. Similarly, if $\chi(X)=0$ the image of $\sigma_{r}^{*}(X) \in$ $L_{r}^{n}\left(\mathbb{Z}\left[\pi_{1}(X)\right]\right)$ is again the absolute torsion $\tau^{\mathrm{NEW}}(X)$.

Example 3.2 The manifold $X=\mathbb{C} P^{2} \# \mathbb{C} P^{2} \# 2\left(S^{1} \times S^{3}\right)$ has a round symmetric signature, whose absolute torsion invariant in $\hat{H}^{0}\left(\mathbb{Z} / 2, K_{1}(\mathbb{Z})\right)$ is non-zero. Its symmetric signature represents a generator of $L_{r h}^{4}(\mathbb{Z})=2 \mathbb{Z}$.

By composing with the augmentation map $\epsilon: \mathbb{Z}\left[\pi_{1}(X)\right] \rightarrow \mathbb{Z}$, setting $\epsilon(g)=1$ for all $g \in \pi_{1}\left(X, x_{0}\right)$, we get a symmetric signature $\sigma^{*}(X) \in L^{n}(\mathbb{Z})$ for any oriented, finite Poincaré complex of dimension $n$ (eg any closed, oriented $n$-manifold). We define the reduced absolute torsion, as the image

$$
\bar{\tau}^{\mathrm{NEW}}(X):=\epsilon_{*}\left(\tau^{\mathrm{NEW}}(X)\right) \in K_{1}(\mathbb{Z})
$$

of the absolute torsion. This invariant is computed from the chain complex of $X$, instead of $\tilde{X}$. Our main result depends on a mod 4 relationship between the ordinary signature of a manifold and the absolute torsion of its symmetric signature over $\mathbb{Z}$. This is an algebraic fact.

Theorem 3.3 Let $(C, \varphi)$ be a finite, based, $4 k$-dimensional algebraic Poincaré complex over $\mathbb{Z}$. Then

$$
\operatorname{sign}(C) \equiv 2 \tau^{\mathrm{NEW}}(C, \varphi)+(2 k+1) \chi(C) \bmod 4
$$

Proof The first step is to show that the right-hand side is an algebraic cobordism invariant for Poincaré complexes over $\mathbb{Z}$. Suppose that $\left(C \oplus C^{\prime} \rightarrow D, \delta \varphi, \varphi \oplus \varphi^{\prime}\right)$ is an algebraic cobordism. Since $\operatorname{dim} C=4 k$, we have

$$
\tau^{\mathrm{NEW}}(C, \varphi)+\tau^{\mathrm{NEW}}\left(C^{\prime},-\varphi^{\prime}\right)=(4 k+1)(2 k+1) \chi(D) \in K_{1}(\mathbb{Z})
$$

by Lemma 2.4. However, $\chi(D)=\frac{1}{2}\left(\chi(C)+\chi\left(C^{\prime}\right)\right) \in \mathbb{Z}$ and $\tau^{\mathrm{NEW}}\left(C^{\prime},-\varphi^{\prime}\right)=$ $\tau^{\mathrm{NEW}}\left(C^{\prime}, \varphi^{\prime}\right)+\chi\left(C^{\prime}\right)$, so that

$$
2 \tau^{\mathrm{NEW}}(C, \varphi)+2 \tau^{\mathrm{NEW}}\left(C^{\prime}, \varphi^{\prime}\right) \equiv(2 k+1) \chi(C)+(2 k-1) \chi\left(C^{\prime}\right) \bmod 4 .
$$

Therefore

$$
2 \tau^{\mathrm{NEW}}(C, \varphi)+(2 k+1) \chi(C) \equiv 2 \tau^{\mathrm{NEW}}\left(C^{\prime}, \varphi^{\prime}\right)+(2 k+1) \chi\left(C^{\prime}\right) \bmod 4
$$

as required (since $\left.2 \chi(C) \equiv 2 \chi\left(C^{\prime}\right) \bmod 4\right)$. Next, we use the fact that every $4 k-$ dimensional symmetric Poincaré complex over $\mathbb{Z}$ is algebraically cobordant to a 
complex $(C, \varphi)$ which is concentrated in dimension $2 k$ (see [22, Proposition 4.5]). In that case $\varphi_{0}$ is an isomorphism, so

$$
\tau^{\mathrm{NEW}}(C, \varphi)=\tau\left(\operatorname{det} \varphi_{0}\right)+\beta(C, C)+\alpha_{4 k}(C)
$$

by Lemma 2.2. But the $\beta$-term is zero in this case, and $\alpha_{4 k}(C)$ contributes $\chi(C)$ when $k$ is odd and zero otherwise. We may express this as $\alpha_{4 k}(C)=k \chi(C) \in K_{1}(\mathbb{Z})$. Therefore

$$
\tau^{\mathrm{NEW}}(C, \varphi)=\tau\left(\operatorname{det} \varphi_{0}\right)+k \chi(C) \in K_{1}(\mathbb{Z})
$$

and the right-hand side becomes

$$
2 \tau^{\mathrm{NEW}}(C, \varphi)+(2 k+1) \chi(C) \equiv 2 \tau\left(\operatorname{det} \varphi_{0}\right)+\chi(C) \equiv \operatorname{det} \varphi_{0}+\chi(C)-1 \bmod 4 .
$$

But there is a classical formula

$$
\operatorname{sign}(\phi) \equiv \operatorname{rank}(\phi)+\operatorname{det}(\phi)-1 \bmod 4
$$

relating the signature and the determinant $\bmod 4$ (see [8, Theorem 3.5]), for any unimodular symmetric bilinear form $\phi$ over the integers. We can apply this to $\varphi_{0}$, and note that $\chi(C)=\operatorname{rank} \varphi_{0}$, since $C$ is concentrated in dimension $2 k$.

Corollary 3.4 If $(C, \varphi)$ is a simple, round Poincaré complex over $\mathbb{Z}$, the signature $\operatorname{sign}(C) \equiv 0 \bmod 4$.

We now consider the case where $X$ is an even-dimensional manifold. Let $\pi^{2}$ denote the subset of $\pi^{a b}$ given by the images of squares from $\pi=\pi_{1}\left(X, x_{0}\right)$.

Proposition 3.5 Let $X$ be a closed, oriented $P L$ manifold of even dimension. Then $\tau^{\mathrm{NEW}}(X) \in \operatorname{Im}\left(\hat{H}^{0}\left(\mathbb{Z} / 2 ; K_{1}(\mathbb{Z})\right) \oplus \hat{H}^{0}\left(\mathbb{Z} / 2 ; \pi^{2}\right) \rightarrow \hat{H}^{0}\left(\mathbb{Z} / 2 ; K_{1}(\mathbb{Z}[\pi])\right)\right)$.

Proof Note that $\hat{H}^{0}\left(\mathbb{Z} / 2 ; \pi^{2}\right)=\pi^{2} \cap\left\{\bar{g} \in \pi^{a b} \mid \bar{g}^{2}=1\right\}$. Suppose that $\tau^{\mathrm{NEW}}(X)=$ $\tau( \pm g)$ for some $g \in \pi$ with image $\bar{g} \neq 1 \in \pi^{a b}$. Then the duality property $\tau^{\mathrm{NEW}}(X)=$ $\tau^{\mathrm{NEW}}(X)^{*}$ implies that $\bar{g}^{2}=1$. Suppose that $\bar{g} \notin \pi^{2}$. Then there is a projection $j: \pi \rightarrow \mathbb{Z} / 2$ such that $j(g)=T$ is the generator of the quotient group $\mathbb{Z} / 2$. Let $p: X^{\prime} \rightarrow X$ denote the 2 -fold covering of $X$ induced by $j$. If $\operatorname{dim} X \equiv 2 \bmod 4$, then

$$
j_{*}\left\{\tau^{\mathrm{NEW}}(X)\right\}=\{\tau( \pm T)\} \in \hat{H}^{0}\left(\mathbb{Z} / 2 ; K_{1}(\mathbb{Z}[\mathbb{Z} / 2])\right)
$$

which is non-zero, contrary to Lemma 2.9 .

If $\operatorname{dim} X=4 k$, then the transfer $p^{!}\left(\tau^{\mathrm{NEW}}(X)\right)=\tau(-1)$, since for finite coverings the fibre bundle transfer is the same as the classical transfer induced by restriction (see [12, page 108]). However, by Theorem B it follows that $\tau^{\mathrm{NEW}}\left(X^{\prime}\right)=p^{!}\left(\tau^{\mathrm{NEW}}(X)\right)$, so we 
have $\bar{\tau}^{\mathrm{NEW}}\left(X^{\prime}\right) \neq 0 \in K_{1}(\mathbb{Z})$. It follows that $\operatorname{sign}\left(X^{\prime}\right) \equiv 2+2 \chi(X) \bmod 4$, from the relation $\chi\left(X^{\prime}\right)=2 \chi(X)$ and Theorem 3.3. The corresponding formula for $X$ gives

$$
\operatorname{sign}(X) \equiv 2 \bar{\tau}^{\mathrm{NEW}}(X)+(2 k+1) \chi(X) \bmod 4
$$

and by the Hirzebruch Signature Theorem we have

$$
\operatorname{sign}\left(X^{\prime}\right)=2 \operatorname{sign}(X) \equiv 2 \chi(X) \bmod 4 .
$$

This is a contradiction, so the covering $X^{\prime} \rightarrow X$ does not exist and $\bar{g} \in \pi^{2}$.

\section{Absolute torsion structures on polyhedra}

In this section we will define the notion of a (pointed) torsion structure on a polyhedron. First we recall some standard definitions (following [3, Section 1]). A polyhedron is a topological space equipped with a maximal family of $P L$ related locally finite triangulations. A cell complex $K$ is a collection of cells $P L$ embedded in a polyhedron $P$ such that

(i) $K$ is a locally finite covering of $P$,

(ii) if $\beta, \gamma \in K$ then $\partial \beta$ and $\beta \cap \gamma$ are unions of cells of $K$ and

(iii) if $\beta, \gamma$ are distinct cells of $K$, then Int $\beta \cap \operatorname{Int} \gamma=\varnothing$.

Let $|K|$ denote the underlying polyhedron of a cell complex, and use $\beta$ to denote a cell in $K$ or the subcomplex it determines. A cell complex $K^{\prime}$ is a subdivision of $K$ if $\left|K^{\prime}\right|=|K|$ and every cell of $K$ is a union of cells of $K^{\prime}$. Any two cell complexes $K^{\prime}, K^{\prime \prime}$ with $\left|K^{\prime}\right|=\left|K^{\prime \prime}\right|$ have a common subdivision $K_{0}$. A base-point for $K$ is a preferred vertex. A pointed map is one which preserves given base-points. A cellular map $h: K \rightarrow L$ between cell complexes is a $P L$ map $h:|K| \rightarrow|L|$ such that for any cell $\beta \in K$, the image $h(\beta)$ is contained in a cell of $L$. Given any continuous map $f:|K| \rightarrow|L|$, there is a subdivision $h: K_{0} \rightarrow K$ and a cellular map $k: K_{0} \rightarrow L$ such that $f \circ h \simeq k$. In other words, any map is homotopic to a cellular map after subdividing the domain. A cellular homeomorphism is a cellular map $h: K \rightarrow L$ such that $h:|K| \rightarrow|L|$ is a homeomorphism. From now on, we will consider only finite cell complexes (those consisting of only finitely many cells), or coverings of finite cell complexes.

If $K$ is a cell complex, we will say that a covering space $\widetilde{P} \rightarrow P$ over $P=|K|$ is subordinate to $K$ if every cell of $K$ is contained in a evenly-covered neighbourhood of $P$. In that case, we let $\widetilde{K}$ be the cell complex on $\widetilde{P}$ induced by the covering $p: \widetilde{P} \rightarrow P$. If $k_{0} \in K$ is a base point and $\pi$ denotes the structural group of the covering (ie there 
is an identification $\left.\pi=p^{-1}\left(k_{0}\right)\right)$, then the cellular chain complex $C(\tilde{K})$ is a chain complex of free $R$-modules, where $R=\mathbb{Z}[\pi]$. We need some additional data to get a chain complex of based modules.

Let $(K, p)$ denote a pointed finite cell complex $K$, with $|K|=P$, and a covering space $p: \widetilde{P} \rightarrow P$ subordinate to $K$, with structural group $\pi$. A geometric basis for $(K, p)$ consists of the following data:

(i) an ordering for the cells of $K$ compatible with the boundary partial ordering,

(ii) an orientation on each cell $\beta \in K$,

(iii) a preferred lift for each cell $\beta \in K$ to $\widetilde{K}$ and

(iv) a sign $\eta_{K} \in \operatorname{Im}\left(K_{1}(\mathbb{Z}) \rightarrow K_{1}(\mathbb{Z}[\pi])\right)$.

We call $(K, p)$ a based cell complex if it is equipped with a geometric basis. Note that the chain complex $C(\widetilde{K})$ of a based cell complex is a signed, based, finite chain complex of finitely-generated free $\mathbb{Z}[\pi]$-modules.

Let $\left(X, x_{0}\right)$ be a pointed space, and let $p: \tilde{X} \rightarrow X$ be a covering space with structural group $\pi$. We fix a base-point $\tilde{x}_{0} \in \tilde{X}$, corresponding to the identity element of $\pi$, with $p\left(\tilde{x}_{0}\right)=x_{0}$.

A pointed torsion structure on $(X, p)$ is a pair $\left(K, c, k_{0}\right)$ such that

(i) $K$ is a pointed finite cell complex, with base-point $k_{0} \in K$,

(ii) $c:|K| \rightarrow X$ is a pointed homeomorphism and

(iii) $\left(K, c^{*}(p)\right)$ is a based cell complex, with the lift of $k_{0}$ mapping to $\tilde{x}_{0}$.

The notation $c^{*}(p):|\widetilde{K}| \rightarrow|K|$ means the pull-back covering via $c$. Usually we will suppress mentioning the base-points and use the notation $(K, c)$.

Definition 4.1 Two pointed torsion structures $\left(K_{1}, c_{1}\right)$ and $\left(K_{2}, c_{2}\right)$ on $(X, p)$ are related by $\left(K_{0}, h, k\right)$ if $K_{0}$ is a pointed cell complex and there exists a homotopy commutative diagram

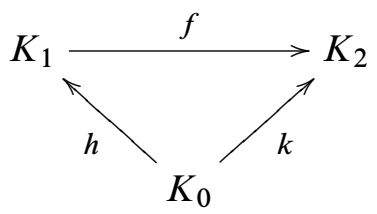

where $h$ is a pointed cellular homeomorphism, $k$ is a pointed cellular map, $f=c_{2}^{-1} \circ c_{1}$, and $f \circ h \simeq k$. 
Unless explicitly mentioned, we assume that all homotopies of pointed maps are base-point preserving. Since $f=c_{2}^{-1} \circ c_{1}$ is homotopic to a cellular map on some subdivision $K_{0}$ of $K$, any two pointed torsion structures are related as above. If $\left(K_{1}, c_{1}\right)$ and $\left(K_{2}, c_{2}\right)$ are related by $\left(K_{0}, h, k\right)$, we let $\left(K_{0}, p_{0}\right)$ denote the pull-back covering $\left(c_{1} \circ h\right)^{*}(p) \cong\left(c_{2} \circ k\right)^{*}(p)$.

Lemma 4.2 Suppose that $\left(K_{1}, c_{1}\right)$ and $\left(K_{2}, c_{2}\right)$ are related by $\left(K_{0}, h, k\right)$, and that $\left(K_{0}, p_{0}\right)$ is a based cell complex. Then the quantity

$$
\tau^{\mathrm{NEW}}\left(K_{1} \leadsto K_{2}\right):=\tau^{\mathrm{NEW}}\left(C\left(\widetilde{K}_{0}\right) \stackrel{k_{*}}{\rightarrow} C\left(\widetilde{K}_{2}\right)\right)-\tau^{\mathrm{NEW}}\left(C\left(\widetilde{K}_{0}\right) \stackrel{h_{*}}{\rightarrow} C\left(\widetilde{K}_{1}\right)\right)
$$

in $K_{1}(\mathbb{Z}[\pi])$ is independent of the choice of $\left(K_{0}, h, k\right)$, and independent of the choice of geometric basis for $\left(K_{0}, p_{0}\right)$.

Proof If we have two choices $\left(K_{0}, h, k\right)$ and $\left(K_{0}^{\prime}, h^{\prime}, k^{\prime}\right)$, there is a homotopy commutative diagram

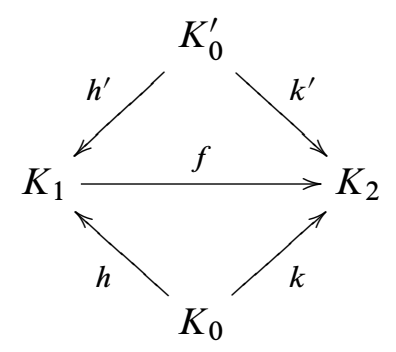

and we apply the chain homotopy invariance of the absolute torsion, and the composition formula of Proposition 1.1, to the composite $C(h)^{-1} \circ C\left(h^{\prime}\right) \simeq C(k)^{-1} \circ C\left(k^{\prime}\right)$ of chain equivalences (and chain homotopy inverses).

Definition 4.3 Two pointed torsion structures $\left(K_{1}, c_{1}\right),\left(K_{2}, c_{2}\right)$ on $(X, p)$ are equivalent if they are related by a based cell complex $\left(K_{0}, p_{0}\right)$ and

$$
\tau^{\mathrm{NEW}}\left(K_{1} \leadsto K_{2}\right)=0 \in K_{1}(\mathbb{Z}[\pi]) \text {. }
$$

Lemma 4.4 This definition gives an equivalence relation on the set of pointed torsion structures on $(X, p)$.

Proof The given relation is reflexive and symmetric. If $\left(K_{1}, c_{1}\right),\left(K_{2}, c_{2}\right)$ are related by $\left(K_{0}, h, k\right)$, and $\left(K_{2}, c_{2}\right),\left(K_{3}, c_{3}\right)$ are related by $\left(K_{0}^{\prime}, h^{\prime}, k^{\prime}\right)$, we have a homotopy 
commutative diagram

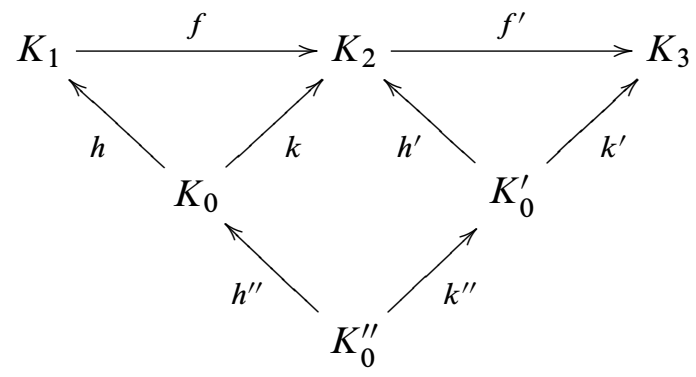

where $\left(K_{0}^{\prime \prime}, h^{\prime \prime}, k^{\prime \prime}\right)$ is the pull-back of $K_{0} \stackrel{k}{\longrightarrow} K_{2} \stackrel{h^{\prime}}{\longleftarrow} K_{0}^{\prime}$. This is a cell complex (the common subdivision of $K_{0}$ and $K_{0}^{\prime}$ ), and $h^{\prime \prime}: K_{0}^{\prime \prime} \rightarrow K_{0}$ is a cellular homeomorphism, so $\left(K_{1}, c_{1}\right)$ and $\left(K_{3}, c_{3}\right)$ are related by $\left(K_{0}^{\prime \prime}, h \circ h^{\prime \prime}, k^{\prime} \circ k^{\prime \prime}\right)$. We choose any geometric basis for $\left(K_{0}^{\prime \prime}, p_{0}^{\prime \prime}\right)$, where $p_{0}^{\prime \prime}$ is the pull-back of the covering $c_{2}^{*}(p)$ by $k \circ h^{\prime \prime} \simeq k^{\prime} \circ h^{\prime \prime}$. It follows that

$$
\tau^{\mathrm{NEW}}\left(K_{1} \leadsto K_{3}\right)=\tau^{\mathrm{NEW}}\left(K_{1} \leadsto K_{2}\right)+\tau^{\mathrm{NEW}}\left(K_{2} \leadsto K_{3}\right)
$$

by the composition formula for the absolute torsion. Therefore $\left(K_{1}, c_{1}\right) \sim\left(K_{2}, c_{2}\right)$ and $\left(K_{2}, c_{2}\right) \sim\left(K_{3}, c_{3}\right)$ implies $\left(K_{1}, c_{1}\right) \sim\left(K_{3}, c_{3}\right)$.

Definition 4.5 Let $\mathcal{S}_{\bullet}(X, p)$ denote the set of equivalence classes of pointed torsion structures on $(X, p)$ for any polyhedron $X$. We will use the notation $[K, c]$ (or just $[K]$ when the reference map is understood) for the equivalence class of a torsion structure $(K, c)$. If the covering space $p$ is understood, we will use the notation $\mathcal{S}_{\bullet}(X)$ for short.

The basic idea of the equivalence relation is to identify two pointed torsion structures whenever the homeomorphism $c_{2}^{-1} \circ c_{1}: K_{1} \rightarrow K_{2}$ is homotopic to a cellular homeomorphism with zero absolute torsion. The more general formulation above will be useful in dealing with subdivisions or amalgamations of cell complexes. We remark that if $K \subset L$ is a subcomplex (containing the base-point), then a pointed torsion structure on $L$ induces a pointed torsion structure on $K$ by restriction of the data.

We now define a pairing

$$
\Phi: \mathcal{S}_{\bullet}(X, p) \times \mathcal{S}_{\bullet}(X, p) \rightarrow K_{1}(\mathbb{Z}[\pi])
$$

by the formula

$$
\Phi([K],[L])=\tau^{\mathrm{NEW}}(K \leadsto L)
$$


where $\left(K, c_{K}\right)$ and $\left(L, c_{L}\right)$ are pointed torsion structures on $(X, p)$ representing the classes $[K]$ and $[L]$ respectively. This is well-defined, and may be computed by taking the absolute torsion of a cellular approximation to $f=c_{L}^{-1} \circ c_{K}$ on a subdivision of $K$. Here are some basic properties of the pairing.

Lemma 4.6 For any pointed torsion structures $[K],[L]$ and $[N]$ on $(X, p)$ :

(i) $\Phi([K],[N])=\Phi([K],[L])+\Phi([L],[N])$,

(ii) $\Phi([K],[L])=-\Phi([L],[K])$ and

(iii) $[K]$ is equivalent to $[L]$ if and only if $\Phi([K],[L])=0$.

Proof The formulas follow directly from the definitions. The details are left to the reader.

\section{Absolute torsion structures on manifolds}

In Theorem 5.2 we give a formula for the absolute torsion of a closed, oriented manifold in terms of the $\Phi$-pairing. This formula will be used in the proof of Theorem B. Our starting point is the fact that any closed $P L$ manifold $X$ of dimension $n$ has a "normal" triangulation (see [27, Section 68]). More precisely, any simplicial $n$-complex $K$ homeomorphic to $X$ has the following properties

(i) each $k$-simplex $(k<n)$ of $K$ is incident with at least one $n$-simplex (this is called a pure $n$-complex),

(ii) each $(n-1)$-simplex of $K$ is incident with exactly two $n$-simplices and

(iii) any two $n$-simplices of $K$ can be connected by a sequence of successively incident simplices of dimensions $n$ and $n-1$.

By subdivision, we can find such a normal triangulation of arbitrarily small diameter. If $X$ is oriented, we can choose a consistent orientation for each $n$-simplex of $K$. In addition, for any element of $\mathcal{S}_{\bullet}(X, p)$ we may find a representative $[K]$ such that the underlying triangulation is normal. There is an analogous cell complex version of this notion, which we call a normal cell structure on $X$.

To compute the absolute torsion of $X$, we will use the classical approach to Poincare duality (see [28, Theorem 2.1], [27, Section 69]). Let $K^{*}$ denote the dual cell complex associated to $K$. This is defined in terms of the triangulation, starting with the first barycentric subdivision $K^{\prime}$ of $K$. Let $\Delta_{r}(K)$ denote the set of $r$-simplices of $K$. The vertices of $K^{\prime}$ are the barycentres $\hat{\sigma}^{r}$ of the simplices $\sigma^{r} \in \Delta_{r}(K)$, and its 
simplices have the form $\left[\hat{\sigma}^{r_{0}}, \hat{\sigma}^{r_{1}}, \ldots, \hat{\sigma}^{r_{s}}\right]$, where $\sigma^{r_{j}}$ is a face of $\sigma^{r_{j+1}}$ for each $j$. Let $\Delta_{r}\left(K^{\prime}\right)$ denote the set of $r$-simplices of $K^{\prime}$. The simplex $\sigma^{r}$ of $K$ is the union of the simplices in $\Delta_{r}\left(K^{\prime}\right)$ which terminate with $\hat{\sigma}^{r}$. The cells $\mathcal{D} \sigma^{r}$ of $K^{*}$ correspond bijectively to the simplices of $K$, and $\mathcal{D} \sigma^{r}$ is the union of the simplices of $K^{\prime}$ which begin with $\hat{\sigma}^{r}$. Then $\operatorname{dim} \mathcal{D} \sigma^{r}=n-r$ and $\sigma^{r}$ intersects $\mathcal{D} \sigma^{r}$ transversely only at $\hat{\sigma}^{r}$. We denote the set of $(n-r)$-cells of $K^{*}$ by $\Delta_{n-r}\left(K^{*}\right)$.

For a suitable chain approximation to the diagonal in $K \times K^{*}$, cap product with the fundamental cycle takes the cochain on $K$ dual to $\sigma^{r}$ to the chain $\mathcal{D} \sigma^{r}$ on $K^{*}$.

Definition 5.1 Let $(K, c)$ be a torsion structure on $X$. We define the dual torsion structure $(\mathcal{D} K, \mathcal{D} c)$ to be the torsion structure on $X$ with underlying cell-complex $\mathcal{D} K$ described above and with geometric basis as follows.

(i) The ordering of the cells on $\mathcal{D} K$ is induced from the ordering of the cells on $K$ : for cells $\mathcal{D} \sigma^{r}$ and $\mathcal{D} \tau^{r}$ of the same dimension, $\mathcal{D} \sigma^{r}$ comes before $\mathcal{D} \tau^{r}$ if and only if $\sigma$ come before $\tau$.

(ii) The lift of each cell of $\mathcal{D} K$ is induced by the lift of the corresponding dual cell.

(iii) The cells of $\mathcal{D} K$ are oriented such that cap product with the fundamental cycle induces the based identity map

$$
\mathcal{D} \varphi_{0}: C(K)^{n-r} \rightarrow C(\mathcal{D} K)_{r} .
$$

With the above choice of geometric basis the chain complex of $\mathcal{D} K$ is $C(\mathcal{D} K)=$

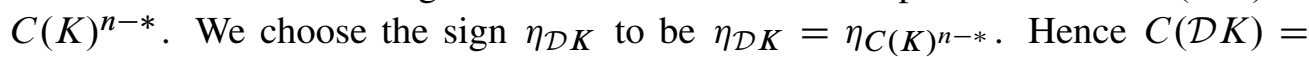
$C(K)^{n-*}$ as signed complexes (identified by $\left.\mathcal{D} \varphi_{0}\right)$.

Theorem 5.2 Let $X$ be a closed, oriented manifold of dimension $n$. Then

$$
\tau^{\mathrm{NEW}}(X)=\Phi([\mathcal{D} K],[K]) \in \widehat{H}^{n}\left(\mathbb{Z} / 2 ; K_{1}\left(\mathbb{Z}\left[\pi_{1}\left(X, x_{0}\right)\right]\right)\right) .
$$

Proof We first choose a geometric basis for the first barycentric subdivision $K^{\prime}$ of $K$. Since $X$ is an oriented manifold we may apply the symmetric construction of Ranicki $[22 ; 23]$ to find a symmetric Poincaré complex $(C(K), \varphi)$; in particular, the map $\varphi_{0}: C(K)^{n-*} \rightarrow C(K)$ is a chain level representative of the Poincaré duality map on $X$ given by cup-product with the fundamental class. We have a diagram of signed chain complexes which commutes up to chain homotopy:

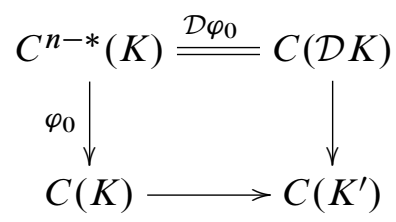


Hence

$$
\begin{aligned}
\tau^{\mathrm{NEW}}(X) & =\tau^{\mathrm{NEW}}\left(\varphi_{0}\right) \\
& =\tau^{\mathrm{NEW}}\left(C(\mathcal{D} K) \rightarrow C\left(K^{\prime}\right)\right)-\tau^{\mathrm{NEW}}\left(C(K) \rightarrow C\left(K^{\prime}\right)\right) \\
& =\Phi\left([\mathcal{D} K],\left[K^{\prime}\right]\right)-\Phi\left([K],\left[K^{\prime}\right]\right) \\
& =\Phi([\mathcal{D} K],[K]) .
\end{aligned}
$$

Thus the theorem is proved.

\section{Fibre transport and transfer}

In this section we will describe a suitable algebraic setting for the fibre transport in a fibre bundle, and establish some useful properties for use in Section 8. Let $\mathbb{A}$ and $\mathbb{B}$ be (small) additive categories with involution, and recall that $S \mathbb{P} \mathbb{D}_{n}(\mathbb{B})$ denotes the full sub-category of $\mathbb{S D}(\mathbb{B})$ consisting of signed $n$-dimensional chain complexes $C$ in $\mathbb{B}$, such that $C^{n-*}$ is chain equivalent to $C$, and $\chi(C)=0$ if $n$ is odd. This sub-category inherits an involution via $C \mapsto C^{n-*}$ (see Definition 12.18).

Definition 6.1 A transfer functor is a set of additive functors $J^{D}: \mathbb{A} \rightarrow \mathbb{S P D}_{n}(\mathbb{B})$, indexed by a set ${ }_{\mathbb{A}} \operatorname{Mod}_{\mathbb{B}}$ of objects $D$ in $\mathbb{S P D}_{n}(\mathbb{B})$ called $\mathbb{A}-\mathbb{B}$ bimodules, such that

(i) if $D \in_{\mathbb{A}} \operatorname{Mod}_{\mathbb{B}}$ and $D \cong D^{\prime}$, then $D^{\prime} \in_{\mathbb{A}} \operatorname{Mod}_{\mathbb{B}}$,

(ii) for each isomorphism $g: D \rightarrow D^{\prime}$ in $\mathbb{S P D}_{n}(\mathbb{B})$ there is a natural transformation $g_{*}: J^{D} \rightarrow J^{D^{\prime}}$, with (id) $)_{*}=$ id and $(h \circ g)_{*}=h_{*} \circ g_{*}$ for any isomorphism $h: D^{\prime} \rightarrow D^{\prime \prime}$ and

(iii) for each object $M \in \mathbb{A}, J^{D}(M)^{*} \cong J^{D^{n-*}}\left(M^{*}\right)$.

If $\left(C, \eta_{C}\right)$ is a signed complex in $\mathbb{A}$, then $J^{D}(C)$ is a signed chain complex in $\mathbb{S P D} \mathbb{D}_{n}(\mathbb{B})$, with $\eta_{J}{ }^{D}(C)=J^{D}\left(\eta_{C}\right)$. This formula gives an allowable sign because

$$
J^{D}(\epsilon(M, N))=\epsilon\left(J^{D}(M), J^{D}(N)\right)
$$

since $J^{D}$ is additive and $\epsilon(M, N)=\tau^{\text {iso }}(M \oplus N \rightarrow N \oplus M)$ for any objects $M, N$ of $\mathbb{A}$. The composition

$$
\operatorname{tr} f_{D}=i_{*} \circ J_{*}^{D}: K_{1}^{\text {iso }}(\mathbb{A}) \rightarrow K_{1}^{\text {iso }}(\mathbb{B})
$$

is called the transfer induced by $J^{D}$.

The main examples for our applications arise from the fibre transport. 
Example 6.2 (Fibre Transport) Let $R$ and $S$ be rings with involution, and let $D$ be an object in $S P \mathbb{D}_{n}(\mathbb{A}(S))$. Suppose that

$$
p: R \rightarrow[D, D]^{o p}
$$

is a homomorphism of rings with involution, where $[D, D]^{o p}$ denotes the set of chain homotopy classes of self-chain maps $D \rightarrow D$ over $S$. Then we let $\mathbb{A}(R) \operatorname{Mod}_{\mathbb{A}(S)}$ be the collection of all objects $D^{\prime} \cong D$. Define

$$
J^{D}: \mathbb{A}(R) \rightarrow \mathbb{P P D}_{n}(\mathbb{A}(S))
$$

on objects by setting $J^{D}(R)=\left(D, \eta_{D}\right)$, and extending additively. As a chain complex, $J^{D}(M)=M \otimes_{R} D$ for each object $M$ in $\mathbb{A}(R)$, and the sign $\eta_{J}{ }_{(M)}$ is determined by the usual formula for direct sums. If $f: M \rightarrow M^{\prime}$ is a morphism in $\mathbb{A}(R)$, then we get an induced morphism

$$
f \otimes 1: M \otimes_{R} D \rightarrow M^{\prime} \otimes_{R} D
$$

where $p$ is used to define a left $R$-module structure on $D$. If $g: D \rightarrow D^{\prime}$ is an isomorphism in $\mathbb{S P D}_{n}(\mathbb{A}(S))$, the natural transformation $g_{*}: J^{D}(C) \rightarrow J^{D^{\prime}}(C)$ is defined by

$$
1 \otimes g: C \otimes_{R} D \rightarrow C \otimes_{R} D^{\prime}
$$

The duality property (iii) follows from the identification $M=R^{m}$ as a based, free $R$-module. The involution $M \mapsto M^{*}$ maps the given base to the dual base. Under these identifications

$$
M^{*} \otimes_{R} D^{n-*}=\oplus_{i=1}^{m} D^{n-*}=\left(\oplus_{i=1}^{m} D\right)^{*}=\left(M \otimes_{R} D\right)^{*} .
$$

In this example, the transfer induced by $J^{D}$ is usually denoted $p^{!}: K_{1}(R) \rightarrow K_{1}(S)$ (see [12]).

As a special case (when $p$ is trivial) we get a transfer functor

$$
J^{D}: \mathbb{A}(R) \rightarrow \mathbb{S P \mathbb { D }} \mathbb{P}_{n}(\mathbb{A}(R \otimes S))
$$

for any two rings with involution (tensor product over $\mathbb{Z}$ ).

Example 6.3 (Tensor products) Let $C$ and $D$ be signed chain complexes over $\mathbb{A}(R)$ and $\mathbb{A}(S)$ respectively, and let $F_{*}(C \otimes D)$ denote the filtered complex structure defined in Example 12.4, Section 12. Then the signed tensor product is the signed filtered complex whose underlying chain complex is $C \otimes D$ and whose associated graded complex is $J^{D}\left(C, \eta_{C}\right)=\left(\mathbb{G}_{*}(C \otimes D), J^{D}\left(\eta_{C}\right)\right)$. The 'internal' sign $\eta_{G_{r}(C \otimes D)}$ is the sign of the chain complex $J^{D}\left(C_{r}\right)$ over $S$, as defined above. In this example, the transfer $p^{!}=\operatorname{tr} f_{D}$ is just multiplication by $\chi(D)$. 
The functorial properties of $J^{D}$ give two useful formulas.

Lemma 6.4 Let $J^{D}: \mathbb{A} \rightarrow \mathbb{S D}_{n}(\mathbb{B})$ be a transfer functor.

(i) For each chain equivalence $f: C \rightarrow C^{\prime}$ in $\mathbb{A}$,

$$
i_{*} \tau^{\mathrm{NEW}}\left(J^{D}(f)\right)=i_{*} J_{*}^{D}\left(\tau^{\mathrm{NEW}}(f)\right)=\operatorname{tr} f_{D}\left(\tau^{\mathrm{NEW}}(f)\right) \in K_{1}^{\text {iso }}(\mathbb{B}) .
$$

(ii) If $J^{D}$ is induced by a fibre transport $R \stackrel{p}{\rightarrow}[D, D]^{o p}$, then for each chain equivalence $g: D \rightarrow D^{\prime}$ in $\mathbb{A}(S)$,

$$
i_{*} \tau^{\mathrm{NEW}}\left(J^{D}(C) \stackrel{g_{*}}{\rightarrow} J^{D^{\prime}}(C)\right)=\chi(C) \cdot \tau^{\mathrm{NEW}}(g) \in K_{1}^{\mathrm{iso}}(\mathbb{A}(S)) .
$$

Proof Part (i) of the Lemma follows directly from the definitions. For Part (ii) observe that $g_{*}: J^{D}(C) \rightarrow J^{D^{\prime}}(C)$ is an isomorphism of chain complexes in $\mathbb{S} \mathbb{D}(\mathbb{A}(S))$ and hence

$$
\begin{aligned}
*^{*} \tau^{\mathrm{NEW}}\left(J^{D}(C) \stackrel{g_{*}}{\rightarrow} J^{D^{\prime}}(C)\right) & =i_{*}\left(\sum(-)^{r} \tau^{\mathrm{iso}}\left(1 \otimes g: C_{r} \otimes_{R} D \rightarrow C_{r} \otimes_{R} D^{\prime}\right)\right) \\
& =\sum(-)^{r} \tau^{\mathrm{NEW}}\left(\bigoplus \operatorname{rank}_{R}\left(C_{r}\right)\left(g: D \rightarrow D^{\prime}\right)\right) \\
& =\chi(C) \tau^{\mathrm{NEW}}\left(g: D \rightarrow D^{\prime}\right) \in K_{1}^{\mathrm{iso}}(\mathbb{A}(S)) .
\end{aligned}
$$

Thus the Lemma is proved.

We now prove the product formula for the absolute torsion of symmetric Poincaré structures announced in [11].

Proposition 6.5 Let $\left(C, \varphi^{C}\right)$ and $\left(D, \varphi^{D}\right)$ be symmetric Poincaré complexes over rings with involution $R$ and $S$ respectively. Then

$$
\tau^{\mathrm{NEW}}\left(C \otimes D, \varphi^{C} \otimes \varphi^{D}\right)=\chi(C) \tau^{\mathrm{NEW}}\left(D, \varphi^{D}\right)+\chi(D) \tau^{\mathrm{NEW}}\left(C, \varphi^{C}\right)
$$

evaluated in $K_{1}(R \otimes S)$.

Proof Let $\operatorname{dim} C=k$ and $\operatorname{dim} D=n$. By definition

$$
\tau^{\mathrm{NEW}}\left(C \otimes D, \varphi^{C} \otimes \varphi^{D}\right)=\tau^{\mathrm{NEW}}\left(\varphi_{0} \otimes \phi_{0}:(C \otimes D)^{n+k-*} \rightarrow(C \otimes D)\right) .
$$

The map $\varphi_{0}^{C} \otimes \varphi_{0}^{D}$ is given by the composition:

$$
(C \otimes D)^{n+k-*} \stackrel{\theta_{C} \otimes D}{\longrightarrow} C^{k-*} \otimes D^{n-*} \stackrel{\varphi_{0} \otimes 1}{\longrightarrow} C \otimes D^{n-*} \stackrel{1 \otimes \varphi_{0}}{\longrightarrow} C \otimes D .
$$

The result now follows from Lemma 6.4 and Lemma 12.27. 


\section{Absolute torsion structures on fibre bundles}

The notion of pointed torsion structures will now be extended to PL or smooth fibre bundles $F^{n} \stackrel{q}{\rightarrow} E^{n+k} \stackrel{p}{\rightarrow} B^{k}$ (see Anderson [1, page 181] for the definition of a PL fibre bundle). We always assume that $F, E$ and $B$ are compatibly oriented PL or smooth, closed manifolds (considered as polyhedra via the canonical $P L$ structure compatible with their smooth structure). In both cases there exists a triangulation of the fibre bundle compatible with a given triangulation on the base (see Putz [21] for the smooth case). The orientation assumption means that $\pi_{1}\left(B, b_{0}\right)$ acts trivially on $H_{n}(F ; \mathbb{Z})$ by fibre transport, and the isomorphism

$$
H_{n+k}(E, \mathbb{Z})=H_{k}(B ; \mathbb{Z}) \otimes H_{n}(F ; \mathbb{Z})
$$

is compatible with the given orientations on total space, base and fibre.

Our goal is to define a notion of a pointed fibre bundle torsion structure (PFBTS) which will have as data a PTS on both the base and fibre, and which will determine a PTS on the total space $E$.

Fix a base-point $b_{0} \in B$ and a base-point $e_{0} \in E$ with $p\left(e_{0}\right)=b_{0}$. We will always assume that $B$ is connected. In the definitions below, the geometric bases for torsion structures on $B$ or $E$ will use their universal covering spaces (suppressed in the notation, together with the chosen lifts of the base-points). In particular, if $c: K \rightarrow B$ is a pointed torsion structure (PTS), and $\gamma \subset \partial \beta$ for some cell $\beta \in K$, then we have a component of the boundary chain map

$$
\partial_{\beta, \gamma}: C(\widetilde{\beta}) \rightarrow S C(\tilde{\gamma})
$$

which can be identified with the module homomorphism

$$
d_{\beta, \gamma}: \mathbb{Z}\left[\pi_{1}\left(B, b_{0}\right)\right] \rightarrow \mathbb{Z}\left[\pi_{1}\left(B, b_{0}\right)\right]
$$

given by multiplication with some element $d_{\beta, \gamma} \in\left\{ \pm \pi^{a b}\right\}$. This is a module isomorphism. We will use the images

$$
p^{!}\left(\tau\left(d_{\beta, \gamma}\right)\right) \in K_{1}\left(\mathbb{Z}\left[\pi_{1}\left(E, e_{0}\right)\right]\right)
$$

under the fibre bundle transfer $p^{!}$in the discussion below.

Let $K \rightarrow B$ and $L \rightarrow E$ be cell structures on the base and total spaces such that the induced map $L \rightarrow K$ is cellular. We call this a cell structure on the fibre bundle. Then we may regard $C(\tilde{L})$ as a filtered complex by defining

$$
F_{r} C(\tilde{L})=C\left(p^{-1}\left(K^{\langle\leqslant r\rangle}\right)\right)
$$


where $K^{\langle\leqslant r\rangle}$ is the subcomplex of $K$ consisting of the cells of dimension $\leqslant r$. If $\operatorname{dim} F=n$ and $\operatorname{dim} B=k$, then we obtain an admissible $(n+k)$-dimensional $k-$ filtered chain complex as defined in Section 12. The associated complex of $F_{*} C(\widetilde{L})$ has the direct sum decomposition

$$
G_{r} C(\tilde{L})=S^{-r} \bigoplus_{\beta \in K^{(r)}} C\left(p^{-1}(\beta)\right)
$$

indexed over the cells in $K$ of dimension $r$. We will use the notation $C\left(\widetilde{F}_{\beta}\right):=$ $S^{-r} C\left(p^{-1}(\beta)\right)$ for $\beta \in K^{(r)}$, so we have the identification

$$
G_{r} C(\tilde{L})=\bigoplus_{\beta \in K^{(r)}} C\left(\tilde{F}_{\beta}\right)
$$

Note that the cell structure on $E$ gives a cell structure on $F_{0}:=p^{-1}\left(b_{0}\right)$. Let $[F]$ denote a pointed torsion structure for $F_{0}$, with respect to the pull-back of the universal covering of $E$ by $q: F \rightarrow E$. Fibre transport defines a ring morphism

$$
\mathbb{Z}\left[\pi_{1} B\right] \rightarrow\left[C\left(\widetilde{F}_{0}\right), C\left(\widetilde{F}_{0}\right)\right]^{o p}
$$

so we have a transfer functor

$$
J^{C\left(\tilde{F}_{0}\right)}: \mathbb{A}\left(\mathbb{Z}\left[\pi_{1} B\right]\right) \rightarrow \mathbb{S P D} \mathbb{D}_{n}\left(\mathbb{A}\left(\mathbb{Z}\left[\pi_{1} E\right]\right)\right) .
$$

To shorten the notation, we will let $J^{F}:=J^{C\left(\widetilde{F}_{0}\right)}$ when the PTS on $F$ is clear from the context.

We can apply this additive functor to the identification

$$
C_{r}(\tilde{K})=\bigoplus_{\beta \in K^{(r)}} C(\tilde{\beta})
$$

to obtain

$$
J^{C\left(\tilde{F}_{0}\right)}\left(C_{r}(\tilde{K})\right)=\bigoplus_{\beta \in K^{(r)}} J^{C\left(\tilde{F}_{0}\right)}(C(\tilde{\beta}))=\bigoplus_{\beta \in K^{(r)}} C\left(\tilde{F}_{0}\right) .
$$

One of our main goals to construct a chain equivalence

$$
\mathcal{E}: \mathbb{G}_{*}(C(\tilde{L})) \rightarrow J^{C\left(\widetilde{F}_{0}\right)} C(\tilde{K})
$$

by constructing chain equivalences

$$
\mathcal{E}^{\beta}: C\left(\widetilde{F}_{\beta}\right) \rightarrow C\left(\widetilde{F}_{0}\right)
$$

for each $\beta \in K$, and defining $\mathcal{E}$ as the direct sum of the $\mathcal{E}^{\beta}$ over all such $\beta$. This can be achieved if we can construct such maps which are compatible with boundary 
inclusions $\gamma<\beta$. We will first construct the maps $\mathcal{E}^{\beta}$ for a special type of PTS on the base $B$ which comes from a normal unfolding (defined below). This construction will then be extended to any PTS on the base $B$.

A pointed torsion structure $c_{K}: K \rightarrow B$ is small with respect to the bundle $E \stackrel{p}{\rightarrow} B$, if $c(\beta)$ is contained in a locally trivial bundle neighbourhood for each $\beta \in K$.

Definition 7.1 A geometric basis for a pointed fibre bundle $\left(E, p, e_{0}\right)$ consists of the following data

(i) a pointed torsion structure $\left(K, c_{K}, k_{0}\right)$ on $B$, which is small with respect to the bundle $(E, p)$,

(ii) a cell structure $L \rightarrow K$ on $E \rightarrow B$, over $c_{K}: K \rightarrow B$ and

(iii) a geometric basis for $F_{\beta} \subset L$, for each $\beta \in K$, with respect to the pull-back of the universal covering of $E$.

Notice that a geometric basis for $(E, p)$ and the fixed base-points give a PTS for the fibre $F$, represented by $\left(F_{0},\left.c_{L}\right|_{F_{0}}\right)$. We denote this pointed torsion structure by $[F] \in \mathcal{S}_{\bullet}(F)$.

Definition 7.2 A pointed fibre bundle torsion structure (PFBTS) on $(E, p)$ consists of a geometric basis $L \rightarrow K$ for $\left.\left(E, p, e_{0}\right)\right)$, such that

(i) for each $\beta \in K$, a chain equivalence $\mathcal{E}^{\beta}: C\left(\widetilde{F}_{\beta}\right) \rightarrow C\left(\widetilde{F}_{0}\right)$ where the map $\mathcal{E}^{b_{0}}: C\left(\widetilde{F}_{0}\right) \rightarrow C\left(\widetilde{F}_{0}\right)$ is the identity,

(ii) for each $\beta \in K$,

$$
\tau^{\mathrm{NEW}}\left(\mathcal{E}^{\beta}: C\left(\widetilde{F}_{\beta}\right) \rightarrow C\left(\widetilde{F}_{0}\right)\right)=0 \in K_{1}\left(\mathbb{Z}\left[\pi_{1}\left(E, e_{0}\right)\right]\right)
$$

and

(iii) the sum

$$
\mathcal{E}=\bigoplus_{\beta \in K} \mathcal{E}^{\beta}: \mathbb{G}_{*}(C(\tilde{L})) \rightarrow J^{C\left(\tilde{F}_{0}\right)} C(\tilde{K})
$$

is a chain isomorphism in $\operatorname{SDD}\left(\mathbb{A}\left(\mathbb{Z}\left[\pi_{1}\left(E, e_{0}\right)\right]\right)\right)$.

We will denote a PFBTS for $(E, p)$ by $(L \rightarrow K, \mathcal{E})$, and the induced PTS on the fibre by $[F]$. We will say that the PFBTS realizes the given structures on base and fibre. 
A PFBTS on $E \rightarrow B$ determines a PTS on $E$ as follows. The choice of geometric basis on each fibre $F_{\beta}$ determines lifts and orientations of each of the cell in $L$. The associated complex $\mathbb{G}_{*}(C(\tilde{L}))$ may be made into a signed complex by setting

$$
G_{r}(C(\tilde{L}))=\bigoplus_{\beta \in K^{\langle r\rangle}} C\left(\tilde{F}_{\beta}\right)
$$

as signed complexes, and setting $\eta_{\mathbb{G}_{*} C(\tilde{L})}=J_{*}^{F}\left(\eta_{K}\right)$. We then give $C(\tilde{L})$ the filtered sign determined by the signs for $\mathbb{G}_{*}(C(\widetilde{L}))$ and define $\eta_{L}=\eta_{F_{*} C(\widetilde{L})}$.

$\operatorname{Lemma~7.3} i_{*} \tau^{\mathrm{NEW}}\left(\mathcal{E}_{*}: \mathbb{G}_{*}(C(\tilde{L})) \rightarrow J^{C\left(\widetilde{F}_{0}\right)} C(\tilde{K})\right)=0 \in K_{1}\left(\mathbb{Z}\left[\pi_{1}\left(E, e_{0}\right)\right]\right)$.

Proof This follows directly from the sign conventions chosen, and the formula

$$
\tau^{\mathrm{NEW}}\left(\mathcal{E}_{*}\right)=\sum_{r}(-1)^{r} \tau^{\text {iso }}\left(\mathcal{E}_{r}: G_{r}(C(\tilde{L})) \rightarrow J^{F} C_{r}(\tilde{K})\right)+\eta_{J^{F} C(\tilde{K})}-\eta_{\mathbb{G}_{*}}(C(\tilde{L}))
$$

for the absolute torsion of a chain isomorphism.

\section{Absolute torsion of fibre bundles}

We will show in Theorem 8.5 that every smooth (or PL) fibre bundle $F \rightarrow E \rightarrow B$ as above admits a pointed fibre bundle pointed torsion structure. First we define the normal unfolding of a normal triangulation of a closed manifold. The idea is to cut $X$ open along certain $(n-1)$-simplices of the triangulation $K$ to obtain a contractible complex projecting onto $X$.

Definition 8.1 Let $K=\bigcup\left\{\Delta_{\alpha}^{n} \mid \alpha \in J_{K}\right\}$, where the $\Delta_{\alpha}^{n}$ are the distinct $n$-simplices in the triangulation of $X$. Let $j_{\alpha}: \Delta_{\alpha}^{n} \rightarrow K$ denote the inclusion maps of the $n-$ simplices. A normal unfolding of $K$ is a pure simplicial $n$-complex $\widehat{K}$ equipped with a simplicial map $f: \widehat{K} \rightarrow K$, such that

(i) $\widehat{K}$ is a union of $n$-simplices, with index set $J_{\widehat{K}}$, and each $(n-1)-$ simplex of $\widehat{K}$ is incident with one or two $n$-simplices,

(ii) there is a bijection $\theta: J_{\widehat{K}} \rightarrow J_{K}$ such that $f \mid \Delta_{\alpha}^{n}=j_{\theta(\alpha)}$ for all $\alpha \in J_{\widehat{K}}$ and

(iii) $\hat{K}$ is contractible.

We again have an analogous notion for normal cell structures.

Lemma 8.2 Let $X$ be a closed smooth manifold of dimension $n$. Each normal cell structure $K$ on $X$ admits a normal unfolding $(\hat{K}, f)$. 
Proof We give the proof for the case of triangulations. Consider the set of barycentres $V=\left\{v_{\alpha} \mid \alpha \in J_{K}\right\}$ of the $n$-simplices of $K$. We can join the barycentres of each pair $\left\{\Delta_{\alpha}^{n}, \Delta_{\beta}^{n}\right\}$ of incident $n$-simplices with an edge $e_{\alpha \beta}$ through their common $(n-1)$ face to obtain a connected graph (the dual 1-skeleton of $K)$. Let $T=(V, E)$ denote a maximal tree in this graph, and note that $T$ has vertex set consisting of all the barycentres. Let $\widehat{K}$ be the quotient space of the disjoint union of the $n$-simplices in $K$, where we identify two $n$-simplices $\Delta_{\alpha}^{n}, \Delta_{\beta}^{n}$ in $\widehat{K}$ along an $(n-1)$ face if and only if $e_{\alpha \beta} \in E$. By construction, $\hat{K}$ is a thickening of the tree $T$, so $\hat{K}$ is contractible. The other properties of a normal unfolding are clear.

A normal unfolding $\widehat{K} \rightarrow K$ can be used to make consistent choices of lifts to the universal covering for simplices in $K$. Any cell $\beta \in K$ is contained in a unique $n$-cell of minimal ordering. We can specify a unique lift $\hat{\beta} \in \hat{K}$ by requiring $\widehat{\beta}$ to be contained in the lift $\widehat{\Delta}$ of $\Delta$.

Definition 8.3 A pointed torsion structure $K \rightarrow B$ is normal if for some normal unfolding $\hat{K} \rightarrow K$, the chosen lifts of adjacent cells $\beta, \gamma \in K$ are adjacent in the universal covering $\widetilde{K}$ whenever their lifts $\widehat{\beta}$ and $\hat{\gamma}$ are adjacent in $\widehat{K}$.

Remark 8.4 If $f: \hat{K} \rightarrow K$ is a normal unfolding, the map $f$ induces an equivalence relation on the $r$-simplices of $\widehat{K}$. Two $r$-simplices are equivalent if they are identified by $f$ to the same simplex in $K$. Let $\partial \hat{K}$ denote the subset of $K$ consisting of all the simplices whose equivalence class contains more than one element. This is a sub-complex of $\hat{K}$ called its boundary.

Let $v_{0}$ denote the barycentre of one of the $n$-simplices (chosen as a base point, so $\left.f\left(v_{0}\right)=k_{0}\right)$, and let $v_{i} \in \tau_{i}$ denote the barycentre of each of the $n$-simplices $\Delta_{i}^{n}$ with a face $\tau_{i} \subset \partial \hat{K}$. Let $x_{i} \in \tau_{i}$ denote the barycentre of each such face, and $e_{i}=\left[v_{i}, x_{i}\right] \subset \Delta_{i}^{n}$ the linear path joining $v_{i}$ to $x_{i}$. Let $\sigma_{i}$ denote the unique path in the maximal tree joining $v_{0}$ with the barycentre $v_{i} \in \Delta_{i}^{n}$, followed by the path $e_{i}$ to $x_{i}$. If $\tau_{i}$ and $\tau_{j}, i<j$, are a pair of $(n-1)$-faces identified under $f: \hat{K} \rightarrow K$, then $f\left(e_{i}\right) \cup f\left(e_{j}\right)=e_{i j}$ is an edge in the dual 1-skeleton of $K$. The fundamental group $\pi_{1}\left(K, k_{0}\right)$ is generated by loops of the form $u_{i j}:=f\left(\sigma_{i}\right) \cup e_{i j} \cup f\left(\sigma_{j}\right)$.

Here is our main result about the existence of pointed fibre bundle torsion structures.

Theorem 8.5 (Realization) Let $E \rightarrow B$ be a fibre bundle of compatibly oriented closed PL manifolds. Given a PTS on $K \rightarrow B$, a cell structure $L \rightarrow K$ on the fibre bundle, and a geometric basis on $F$, there exists a PFBTS $(L \rightarrow K, \mathcal{E})$ realizing this data. 
The main steps in the proof are contained in the following special case.

Proposition 8.6 There exists a PFBTS $(L \rightarrow K, \mathcal{E})$ on $E \rightarrow B$ realizing a given normal pointed torsion structure $K \rightarrow B$ on $B$, a given cell structure $L \rightarrow K$ on the fibre bundle, and a given geometric basis for $F$.

Proof We are required to construct chain maps $\mathcal{E}^{\beta}: C\left(\widetilde{F}_{\beta}\right) \rightarrow C\left(\widetilde{F}_{0}\right)$ such that the direct sum $\mathcal{E}: \mathbb{G}_{*}(C(\widetilde{L})) \rightarrow J^{F} C(\widetilde{K})$ is a chain equivalence. Once these maps are constructed, we can choose a geometric basis on each $F_{\beta}$ such that the maps $\mathcal{E}^{\beta}: C\left(\widetilde{F}_{\beta}\right) \rightarrow C\left(\widetilde{F}_{0}\right)$ have trivial absolute torsion (this is condition (ii) for a PFBTS). The condition that $\mathcal{E}$ is a chain map is equivalent to the statement that for a pair of simplices $\gamma<\beta$ where $\gamma$ is an $(r-1)$-dimensional cell in the boundary of an $r$-dimensional cell $\beta$, the diagram

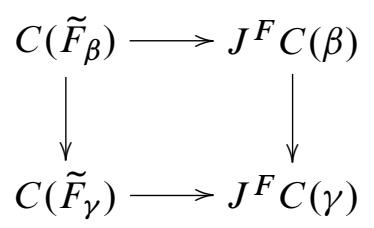

commutes. We will first construct the maps $\mathcal{E}$ and then show that this diagram commutes for all $\gamma<\beta$.

Let $f: \widehat{K} \rightarrow K$ be the normal unfolding of $K$, and $g: \hat{L} \rightarrow L$ the pull-back of $L \rightarrow K$ over $\hat{K}$. The chain complex $\mathbb{G}_{*}(C(\hat{L}))$ splits over the cells $\hat{\beta} \in \hat{K}$ as:

$$
\mathbb{G}_{*}(C(\hat{L}))=\bigoplus_{\hat{\beta} \in \hat{K}} C\left(\widetilde{F}_{\hat{\beta}}\right) .
$$

We assume that the base-point $k_{0}$ is uniquely covered $\left(f^{-1}\left(k_{0}\right)=v_{0}\right)$. The chain complex $C(\hat{K})$ is a based $\mathbb{Z}$-module chain complex since we have an orientation for each cell. Since $\widehat{K}$ is contractible the pull-back of the universal cover of $B$ over the map $\widehat{K} \rightarrow K \rightarrow B$ is trivial and hence we have a chain map

$$
f_{*}: C(\widehat{K}) \otimes_{\mathbb{Z}} \mathbb{Z}\left[\pi_{1} B\right] \rightarrow C(\tilde{K})
$$

of based signed chain complexes over $\mathbb{Z}\left[\pi_{1} B\right]$. On the total space we have a filtered chain map $g_{*}: C(\hat{L}) \rightarrow C(\widetilde{L})$ whose associated map

$$
\mathbb{G}_{*}\left(g_{*}\right): \mathbb{G}_{*}(C(\widehat{L})) \rightarrow \mathbb{G}_{*}(C(\tilde{L}))
$$

splits over each cell $\beta$ of $K$, and lifts $\hat{\beta}$ of $\beta$ giving an isomorphism of chain complexes:

$$
g_{\widehat{\beta}}: C\left(\widetilde{F}_{\hat{\beta}}\right) \rightarrow C\left(\widetilde{F}_{\beta}\right)
$$


Note that

$$
J^{F}\left(C(\hat{K}) \otimes_{\mathbb{Z}} \mathbb{Z}\left[\pi_{1} B\right]\right)=C(\widehat{K}) \otimes_{\mathbb{Z}} C\left(\widetilde{F}_{0}\right) .
$$

Since $\widehat{K}$ is contractible and the triangulation $K \rightarrow B$ is small with respect to the bundle $E \rightarrow B$, there is a trivializing homeomorphism

$$
\Psi_{K}:|\widehat{L}| \rightarrow|\widehat{K}| \times F
$$

of the pull-back bundle $\hat{p}: \hat{L} \rightarrow \widehat{K}$ which gives rise to a chain homotopy commutative diagram of chain maps:

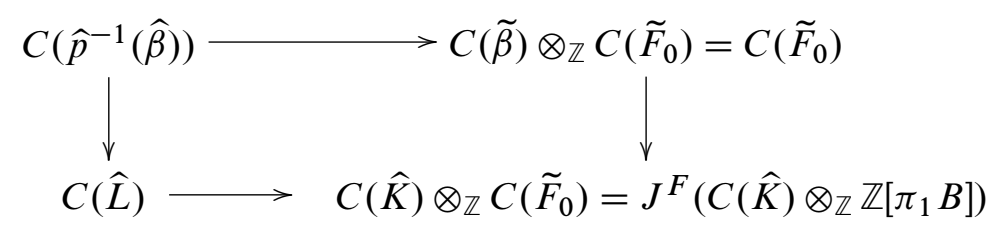

for each $\hat{\beta} \in \hat{K}$. Let $r$ be the dimension of $\beta$. By taking the associated complexes and maps in this diagram, we construct maps $\mathcal{E}^{\widehat{\beta}}$ :

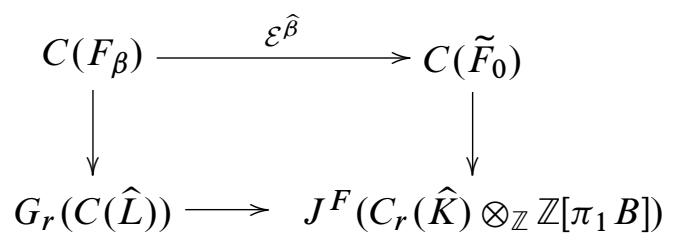

The sum $\widehat{\mathcal{E}}: \mathbb{G}_{*}(C(\hat{L})) \rightarrow J^{C\left(\widetilde{F}_{0}\right)}\left(C(\hat{K}) \otimes_{\mathbb{Z}} \mathbb{Z}\left[\pi_{1} B\right]\right)$ is a chain map and each $\mathcal{E}^{\widehat{\beta}}$ is a chain equivalence.

Each cell $\beta$ in $K$ lies in an $n$-simplex of minimal ordering so we have a particular lift $\hat{\beta}$ of $\beta$. We define the chain equivalence $\mathcal{E}_{\beta}$ to be

$$
\mathcal{E}^{\beta}=\mathcal{E}^{\widehat{\beta}} \circ\left(g_{\widehat{\beta}}\right)^{-1}: C\left(\widetilde{F}_{\beta}\right) \rightarrow C\left(\widetilde{F}_{\widehat{\beta}}\right) \rightarrow C\left(\widetilde{F}_{0}\right)
$$

where $g_{\widehat{\beta}}$ is the restriction of $g: \widehat{L} \rightarrow L$ to $\widehat{\beta}$. It remains to show that $\mathcal{E}_{*}$ is a chain map, in other words that the diagram (2) commutes for all pairs $\beta, \gamma$. This is true if the lifts of the cells $\beta$ and $\gamma$ are adjacent in $\widehat{K}$, since we have a commutative diagram:

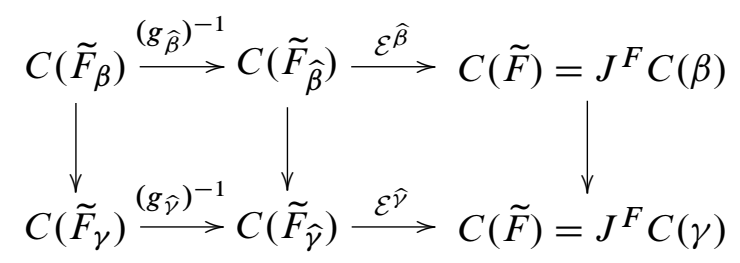


Suppose now that the lift of $\gamma$ is not adjacent to that of $\beta$. Then the component of the differential in $C(\widetilde{K})$ given by $C(\beta) \rightarrow C(\gamma)$ is $d_{\beta, \gamma}= \pm t$ for some non-trivial group element $t \in \pi_{1} B$, when considered as a map of based complexes. We will denote by \pm the sign in front of $t$. Let $\hat{\gamma}^{\prime}$ be the lift of $\gamma$ which is adjacent to the chosen lift of $\hat{\beta}$ of $\beta$. The two faces $\hat{\gamma}, \hat{\gamma}^{\prime}$ are identified under $\widehat{K} \rightarrow K$. Let $f_{\widehat{\gamma}}, f_{\widehat{\gamma}^{\prime}}$ be paths in $\hat{K}$ joining the barycentres $x_{\hat{\gamma}}, x_{\hat{\gamma}^{\prime}}$ with the base point $v_{0}$. Then the image of $f_{\widehat{\gamma}} \cup f_{\widehat{\gamma}^{\prime}}$ in $K$ is a loop representing $t$. Hence fibre transport around $t$ is described by the following composition:

$$
F \rightarrow F \times x_{\widehat{\gamma}} \stackrel{\left.\Psi\right|_{x_{\widehat{\gamma}}}}{\longrightarrow} \hat{p}^{-1}\left(x_{\widehat{\gamma}}\right) \rightarrow p^{-1}\left(x_{\gamma}\right) \rightarrow \hat{p}^{-1}\left(x_{\widehat{\gamma}^{\prime}}\right) \stackrel{\left(\left.\Psi\right|_{x_{\hat{\gamma}^{\prime}}}\right)^{-1}}{\longrightarrow} F \times x_{\hat{\gamma}^{\prime}} \rightarrow F
$$

where $x_{\gamma}$ is the barycentre of $\gamma$ in $K$. Therefore the composition of homotopy equivalences:

$$
F \rightarrow F \times|\widehat{\gamma}| \stackrel{\left.\Psi_{K}\right|_{\hat{\gamma}}}{\longrightarrow} F_{\widehat{\gamma}} \stackrel{\left.g\right|_{\gamma}}{\longrightarrow} F_{\gamma} \stackrel{\left(\left.g\right|_{\gamma^{\prime}}\right)^{-1}}{\longrightarrow} F_{\widehat{\gamma}^{\prime}} \stackrel{\left(\left.\Psi_{K}\right|_{\hat{\gamma}^{\prime}}\right)^{-1}}{\longrightarrow} F \times\left|\hat{\gamma}^{\prime}\right| \rightarrow F
$$

is described up to homotopy by fibre transport around $t$. Hence the composition

$$
C(\widetilde{F}) \stackrel{\left(\mathcal{E}^{\widehat{\gamma}}\right)^{-1}}{\longrightarrow} C\left(\widetilde{F}_{\widehat{\gamma}}\right) \stackrel{g_{\widehat{\gamma}}}{\longrightarrow} C\left(\widetilde{F}_{\gamma}\right) \stackrel{\left(g_{\widehat{\gamma}^{\prime}}\right)^{-1}}{\longrightarrow} C\left(\widetilde{F}_{\widehat{\gamma}^{\prime}}\right) \stackrel{\mathcal{E}^{\hat{\mathcal{\gamma}}^{\prime}}}{\longrightarrow} C(\widetilde{F})
$$

is given (up to chain homotopy) by $J^{F}(t): C(\widetilde{F}) \rightarrow C(\widetilde{F})$. From this we deduce that

$$
\mathcal{E}^{\gamma}=J^{F}(t) \circ\left(\mathcal{E}^{\widehat{\gamma}^{\prime}} \circ\left(g_{\widehat{\gamma}^{\prime}}\right)^{-1}\right)
$$

Since $\hat{\gamma}^{\prime}$ is adjacent to $\hat{\beta}$ we know that

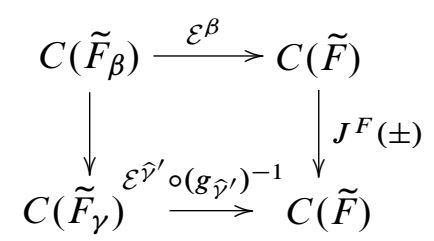

commutes. It now follows that

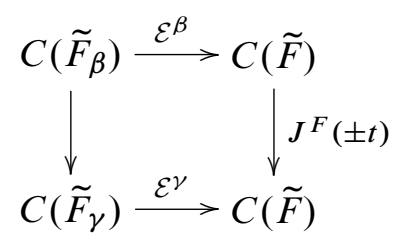

commutes as required. 
The proof of Theorem 8.5 Let $K^{\prime} \rightarrow B$ be a normal PTS on $B$ with the same underlying cell-structure as $K$. Then we can find a PFBTS $\left(L^{\prime} \rightarrow K^{\prime}, \mathcal{E}^{\prime}\right)$ with $L^{\prime}$ having the same underlying cell structure as $L$ and fibre structure $F$ by the above proposition. For each cell $\beta \in K$ we have a corresponding cell $\beta^{\prime} \in K^{\prime}$ and an identification of $C\left(F_{\beta}\right)$ with $C\left(F_{\beta^{\prime}}\right)$. We use this identification to choose a geometric basis for $F_{0}$ such that $C\left(F_{k_{0}}\right) \rightarrow C\left(F_{k_{0}^{\prime}}\right)$ is the based identity map. We now define the maps $\mathcal{E}^{\beta}$ by

$$
\mathcal{E}^{\beta}=J^{F}\left(C\left(\beta^{\prime}\right) \rightarrow C(\beta)\right) \circ\left(\mathcal{E}^{\prime}\right)^{\beta^{\prime}}: C\left(\widetilde{F}_{\beta}\right) \rightarrow C\left(\widetilde{F}_{0}\right)
$$

using the identification of $C\left(F_{\beta}\right)$ with $C\left(F_{\beta^{\prime}}\right)$. We again choose a geometric basis on each $F_{\beta}$ such that the maps $\mathcal{E}^{\beta}: C\left(\widetilde{F}_{\beta}\right) \rightarrow C\left(\widetilde{F}_{0}\right)$ have trivial absolute torsion. We claim that $(L \rightarrow K, \mathcal{E})$ is a PFBTS. The only condition remaining to check is that $\mathcal{E}$ is a chain map. However, by construction, $\mathcal{E}$ is the composition

$$
\mathbb{G}_{*}(C(\tilde{L})) \rightarrow \mathbb{G}_{*}\left(C\left(\tilde{L}^{\prime}\right)\right) \stackrel{\mathcal{E}^{\prime}}{\rightarrow} J^{F} C\left(\tilde{K}^{\prime}\right) \stackrel{J^{F}\left(C\left(\tilde{K}^{\prime}\right) \rightarrow C(\tilde{K})\right)}{\longrightarrow} J^{F} C(\tilde{K})
$$

of chain maps and hence is itself a chain map.

Remark 8.7 The maps $\mathcal{E}^{\beta}$ in a PFBTS have a geometric interpretation. For an oriented cell $\beta \in K$, the intersection of cells of $p^{-1}(\beta)$ with $p^{-1}(\hat{\beta})$, the barycentre of $\beta$, defines a cell-structure on $p^{-1}(\hat{\beta})$. A lift of a cell in $p^{-1}(\beta)$ determines a lift of the corresponding cell in $p^{-1}(\widehat{\beta})$, and also the orientation of the cell in $p^{-1}(\beta)$ along with the orientation of $\beta$ determines an orientation of the cell in $p^{-1}(\widehat{\beta})$. This determines a PTS on $p^{-1}(\hat{\beta})$; moreover we have an identification of based chain complexes:

$$
C\left(\widetilde{F}_{\beta}\right) \rightarrow C\left(p^{-1}(\hat{\beta})\right) .
$$

We can regard the choice of lift of $\beta$ as a path $\sigma$ from the base point $b_{0}$ to $\beta$. Then the map $\mathcal{E}^{\beta}$ composed with the above identification

$$
\mathcal{E}^{\beta}: C\left(p^{-1}(\hat{\beta})\right) \rightarrow C(\tilde{F})
$$

is a chain level representative of the map from $p^{-1}(\widehat{\beta})$ to $F$ given by fibre transport along $\sigma$.

\section{The proof of Theorem B}

In order to prove Theorem B we will need to show how the absolute torsions computed from a pointed torsion structure on a fibre bundle vary when we change the PTS on the base or on the fibre. Let $\mathcal{S}_{\bullet}(F \stackrel{q}{\rightarrow} E)$ denote the set of pointed torsion structures 
on $F_{0}=p^{-1}\left(b_{0}\right)$ with respect to the pull-back of the universal covering of $E$. We summarize the results in the following Proposition.

Proposition 9.1 Given $[B] \in \mathcal{S}_{\bullet}(B)$ and $[F] \in \mathcal{S}_{\bullet}(F \stackrel{q}{\rightarrow} E)$, there exists a fibre bundle torsion structure $(L \rightarrow K, \mathcal{E})$ on $F \rightarrow E \rightarrow B$, such that $[K]=[B]$ and the class of the fibre structure is $[F]$. Moreover any two such fibre bundle torsion structures represent the same element in $\mathcal{S}_{\bullet}(E)$.

Let $\mathfrak{E}_{[B][F]} \in \mathcal{S}_{\bullet}(E)$ denote the induced torsion structure on the total space.

Proposition 9.2 Let $\left[B_{1}\right],\left[B_{2}\right] \in S_{\bullet}(B)$ be any two torsion structures for the base, and let $\left.\left[F_{1}\right],\left[F_{2}\right] \in \mathcal{S}_{\bullet}(F \stackrel{q}{\rightarrow} E)\right)$ be any two fibre structures. Then

$$
\Phi\left(\mathfrak{E}_{\left[B_{1}\right]\left[F_{1}\right]}, \mathfrak{E}_{\left[B_{2}\right]\left[F_{2}\right]}\right)=p^{!} \Phi\left(\left[B_{1}\right],\left[B_{2}\right]\right)+\chi(B) \Phi\left(\left[F_{1}\right],\left[F_{2}\right]\right) .
$$

The dual torsion structure $\mathcal{D} \mathfrak{E}_{[B][F]}$ of $\mathfrak{E}_{[B][F]}$ was defined in Definition 5.1.

Proposition 9.3 Let $\mathcal{D} \mathfrak{E}_{[B][F]}$ denote the dual torsion structure of $\mathfrak{E}_{[B][F]}$ in $\mathcal{S}_{\bullet}(E)$. Then

$$
\Phi\left(\mathcal{D} \mathfrak{E}_{[B][F]}, \mathfrak{E}_{[\mathcal{D} B][\mathcal{D} F]}\right)=0 .
$$

These properties will be established in a sequence of lemmas.

Lemma 9.4 Given PTS's on $K$ and $F$ the maps $\mathcal{E}^{\beta}: C\left(\widetilde{F}_{\beta}\right) \rightarrow C\left(\widetilde{F}_{0}\right)$ are uniquely determined by the properties:

(i) the map $\mathcal{E}^{b_{0}}: C\left(\widetilde{F}_{0}\right) \rightarrow C\left(\widetilde{F}_{0}\right)$ is the identity and

(ii) the direct sum $\mathcal{E}=\bigoplus_{\beta} \mathcal{E}^{\beta}$ is a chain equivalence.

Proof Suppose that $\left\{\mathcal{E}_{1}^{\beta}\right\}$ and $\left\{\mathcal{E}_{2}^{\beta}\right\}$ are two sets of chain maps satisfying these conditions. Since $B$ is connected, any cell $\beta \in K$ can be connected to the base-point by a sequence of adjacent pairs of cells. If $\gamma \subset \partial \beta$, we have a commutative diagram

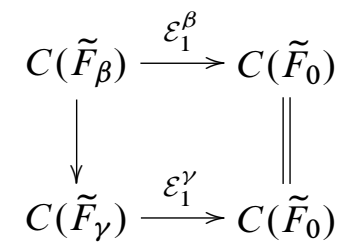

and similarly for $\mathcal{E}_{2}$. We are using the identification $J^{F} C(\beta)=C\left(\widetilde{F}_{0}\right)$, valid for every cell. But the horizontal maps are isomorphisms in $\operatorname{SPD}_{n}\left(\mathbb{Z}\left[\pi_{1}\left(E, e_{0}\right)\right]\right)$, so $\mathcal{E}_{1}^{\beta}=\mathcal{E}_{2}^{\beta}$ if and only if $\mathcal{E}_{1}^{\gamma}=\mathcal{E}_{2}^{\gamma}$. However, these two maps agree at the base-point $b_{0}$, so they agree over every cell and $\mathcal{E}_{1}=\mathcal{E}_{2}$. 
Lemma 9.5 Suppose that $\left(L_{1} \rightarrow K, \mathcal{E}_{1}\right)$ and $\left(L_{2} \rightarrow K, \mathcal{E}_{2}\right)$ are pointed fibre bundle torsion structures on $E \rightarrow B$ with fibre structures $F_{1}$ and $F_{2}$ respectively. If $h: L_{1} \rightarrow$ $L_{2}$ is a subdivision over $K$, then there is a commutative diagram

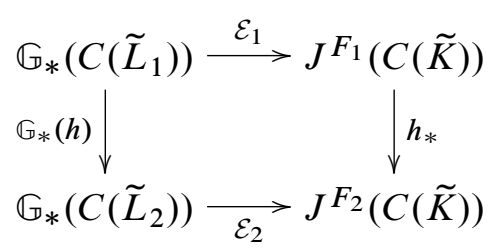

in $\mathbb{S P D} \mathbb{D}_{n}\left(\mathbb{Z}\left[\pi_{1}\left(E, e_{0}\right)\right]\right)$.

Proof Since any subdivision over $K$ is a filtered map, we can pass to the associated graded complexes. The composition

$$
\mathcal{E}_{1}^{\prime}: \mathbb{G}_{*}\left(C\left(\tilde{L}_{1}\right)\right) \stackrel{\mathbb{G}_{*}(h)}{\longrightarrow} \mathbb{G}_{*}\left(C\left(\tilde{L}_{2}\right)\right) \stackrel{\mathcal{E}_{2}}{\longrightarrow} J^{F_{2}} C(\tilde{K}) \stackrel{\left(h_{*}\right)^{-1}}{\longrightarrow} J^{F_{1}} C(\tilde{K})
$$

splits over each simplex $\beta \in K$ to the composition:

$$
\mathcal{E}_{1}^{\prime \beta}: C\left(\tilde{F}_{1 \beta}\right) \stackrel{\mathbb{G}_{*}\left(\left.h_{*}\right|_{\beta}\right)}{\longrightarrow} C\left(\tilde{F}_{2 \beta}\right) \stackrel{\mathcal{E}_{2}^{\beta}}{\longrightarrow} J^{F_{2}} C(\beta) \stackrel{\left(h_{*}\right)^{-1}}{\longrightarrow} J^{F_{1}} C(\beta) .
$$

In particular the map $\mathcal{E}_{1}^{\prime b_{0}}$ coincides with $\mathcal{E}_{1}{ }^{b_{0}}$, so the above remark implies that $\mathcal{E}_{1}^{\prime}=\mathcal{E}_{1}$. Hence the diagram commutes.

Corollary 9.6 Suppose that $\left(L_{1} \rightarrow K, \mathcal{E}_{1}\right)$ and $\left(L_{2} \rightarrow K, \mathcal{E}_{2}\right)$ are pointed fibre bundle torsion structures on a fibre bundle $F \rightarrow E \rightarrow B$. The induced pointed torsion structures $\left[L_{1}\right]$ and $\left[L_{2}\right]$ on $E$ satisfy

$$
\Phi\left(\left[L_{1}\right],\left[L_{2}\right]\right)=\chi(B) \Phi\left(\left[F_{1}\right],\left[F_{2}\right]\right) .
$$

Proof If $L_{2}$ is a subdivision of $L_{1}$, then the commutativity of the diagram implies that

$$
\tau^{\mathrm{NEW}}\left(\mathbb{G}_{*}\left(C\left(\tilde{L}_{1}\right)\right) \rightarrow \mathbb{G}_{*}\left(C\left(\tilde{L}_{2}\right)\right)\right)=\tau^{\mathrm{NEW}}\left(J^{F_{1}}(C(\tilde{K})) \rightarrow J^{F_{2}}(C(\tilde{K}))\right)
$$

since the maps $\mathcal{E}_{1}$ and $\mathcal{E}_{2}$ have trivial absolute torsion. By Lemma 6.4

$$
\begin{aligned}
i_{*} \tau^{\mathrm{NEW}}\left(\mathbb{G}_{*}\left(C\left(\tilde{L}_{1}\right)\right) \rightarrow \mathbb{G}_{*}\left(C\left(\tilde{L}_{2}\right)\right)\right) & =\chi(B) \tau^{\mathrm{NEW}}\left(C\left(\tilde{F}_{1}\right) \rightarrow C\left(\tilde{F}_{2}\right)\right) \\
& =\chi(B) \Phi\left(\left[F_{1}\right],\left[F_{2}\right]\right) .
\end{aligned}
$$


From Theorem 12.17 on the torsion of filtered maps we see that:

$$
\begin{aligned}
\Phi\left(\left[L_{1}\right],\left[L_{2}\right]\right) & =\tau^{\mathrm{NEW}}\left(C\left(\widetilde{L}_{1}\right) \rightarrow C\left(\tilde{L}_{2}\right)\right) \\
& =i_{*} \tau^{\mathrm{NEW}}\left(\mathbb{G}_{*}\left(C\left(\tilde{L}_{1}\right)\right) \rightarrow \mathbb{G}_{*}\left(C\left(\tilde{L}_{2}\right)\right)\right) \\
& =\chi(B) \Phi\left(\left[F_{1}\right],\left[F_{2}\right]\right)
\end{aligned}
$$

as required. We now consider the general case where $L_{2}$ is not necessarily a subdivision of $L_{1}$; in this case we may find a filtered cellular map $L_{3} \rightarrow L_{1}$ which is a common subdivision (as cell-complexes) of $L_{1}$ and $L_{2}$. By Theorem 8.5 we may find a PFBTS whose underlying triangulation is $L_{3}$, we will also denote this by $L_{3}$. It now follows that

$$
\begin{aligned}
\Phi\left(\left[L_{1}\right],\left[L_{2}\right]\right) & =\Phi\left(\left[L_{2}\right],\left[L_{3}\right]\right)-\Phi\left(\left[L_{1}\right],\left[L_{3}\right]\right) \\
& =\chi(B) \Phi\left(\left[F_{2}\right],\left[F_{3}\right]\right)-\chi(B) \Phi\left(\left[F_{1}\right],\left[F_{3}\right]\right) \\
& =\chi(B) \Phi\left(\left[F_{1}\right],\left[F_{2}\right]\right)
\end{aligned}
$$

as required.

Lemma 9.7 Suppose that $\left(L_{1} \rightarrow K_{1}, \mathcal{E}_{1}\right)$ and $\left(L_{2} \rightarrow K_{2}, \mathcal{E}_{2}\right)$ are pointed fibre bundle torsion structures on $E \rightarrow B$. Let $h: L_{1} \rightarrow L_{2}, f: K_{1} \rightarrow K_{2}$ be cellular homeomorphisms, with $p_{L_{2}} \circ h=f \circ p_{L_{1}}$ and $h$ inducing the identity on $F=p^{-1}\left(b_{0}\right)$. If both PFBTS's have the same fibre structure under this identification, then there is a commutative diagram

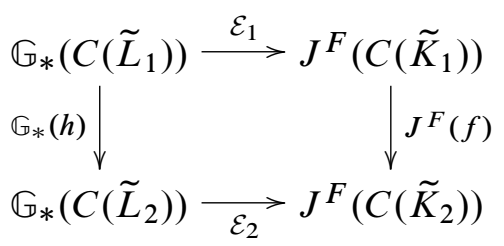

in $\mathbb{S D}_{n}\left(\mathbb{Z}\left[\pi_{1}\left(E, e_{0}\right)\right]\right)$.

Proof We first consider the case where $K_{1}$ and $K_{2}$ differ only by the choice of PTS. Then the composition

$$
\mathbb{G}_{*}\left(C\left(\tilde{L}_{1}\right)\right) \stackrel{\mathbb{G}_{*}(h)}{\longrightarrow} \mathbb{G}_{*}\left(C\left(\tilde{L}_{2}\right)\right) \stackrel{\mathcal{E}_{2}}{\longrightarrow} J^{F} C\left(\tilde{K}_{2}\right) \stackrel{J^{F}\left(f_{*}^{-1}\right)}{\longrightarrow} J^{F} C\left(\tilde{K}_{1}\right)
$$

splits over the cells of $K_{1}=K_{2}$ to give maps $\mathcal{E}^{\prime \beta}: \mathbb{G}_{*}\left(C\left(\widetilde{F}_{\beta}\right)\right) \rightarrow J^{F} C\left(\widetilde{K}_{1}\right)$. However Lemma 9.4 shows that these maps must coincide with the $\mathcal{E}_{1}^{\beta}$, so the diagram commutes in this case. 
It is now sufficient to consider the case where the map $K_{1} \rightarrow K_{2}$ is an elementary subdivision of one cell $\beta$ and where $L_{1} \rightarrow L_{2}$ is a cellular isomorphism away from $p^{-1}(\beta)$. We may also assume the geometric basis for $K_{2}$ is the same as that for $K_{1}$ away from $\beta$ and that the geometric basis for each $C\left(\widetilde{F}_{1 \gamma}\right)$ coincides with that for $C\left(\widetilde{F}_{2 \gamma}\right)$ for $\gamma \neq \beta$. We may also assume that the maps $\mathcal{E}_{1}^{\gamma}$ and $\mathcal{E}_{2}^{\gamma}$ are equal for all $\gamma \neq \beta$ so it is sufficient to show that the diagram commutes on the $C\left(\tilde{F}_{\beta}\right)$ factor. Let $\left\{\beta_{i}\right\}_{i}$ be the components of the subdivision of $\beta$ in $K_{2}$ which have the same dimension as $\beta$. Then we must show that the diagram

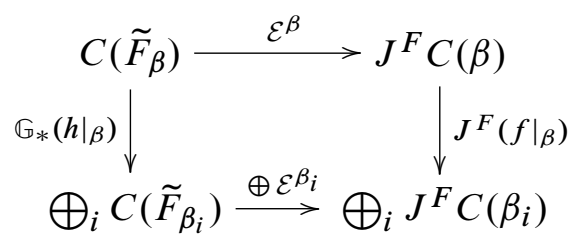

commutes. The RHS of the diagram splits over the components $C\left(\widetilde{F}_{\sigma_{i}}\right)$ so it is sufficient to show that

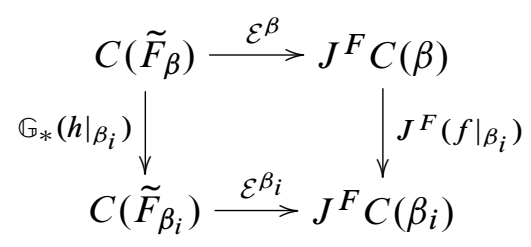

commutes for each $i$. Let $\tau$ be a boundary component of both $\beta_{i}$ and $\beta$ (such a component can always be found for an elementary subdivision). Then we have a diagram of chain equivalences:

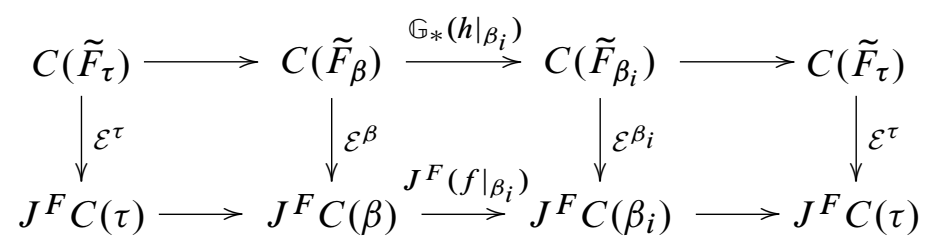

The outer square commutes since the compositions along the top and bottom are the identity maps. The left and right squares commute since both $\mathcal{E}_{1}$ and $\mathcal{E}_{2}$ are chain maps. Hence the middle square commutes as required.

Corollary 9.8 Let $K_{1}$ and $K_{2}$ be cell-decompositions of $B$; let $L_{1} \rightarrow K_{1}$ and $L_{2} \rightarrow K_{2}$ be PFBTS's on $E \rightarrow B$ with fibre class [F]. Then the induced PTS's on $E$, $\left[L_{1}\right]$ and $\left[L_{2}\right]$ satisfy

$$
\Phi\left(\left[L_{1}\right],\left[L_{2}\right]\right)=p^{!} \Phi\left(\left[K_{1}\right],\left[K_{2}\right]\right)
$$


Proof If $L_{2}$ and $K_{2}$ are subdivisions of $L_{1}$ and $K_{1}$ respectively, then commutativity of the diagram of the above lemma and Lemma 6.4 give:

$$
\begin{aligned}
i_{*} \tau^{\mathrm{NEW}}\left(\mathbb{G}_{*}\left(C\left(\tilde{L}_{1}\right)\right) \rightarrow \mathbb{G}_{*}\left(C\left(\widetilde{L}_{2}\right)\right)\right) & =\tau^{\mathrm{NEW}}\left(J^{F} C\left(\widetilde{K}_{1}\right) \rightarrow J^{F} C\left(\widetilde{K}_{2}\right)\right) \\
& =p^{!} \tau^{\mathrm{NEW}}\left(C\left(\widetilde{K}_{1}\right) \rightarrow C\left(\widetilde{K}_{2}\right)\right) .
\end{aligned}
$$

Using Theorem 12.17 on the torsion of filtered maps we see that:

$$
\begin{aligned}
\Phi\left(\left[L_{1}\right],\left[L_{2}\right]\right) & =\tau^{\mathrm{NEW}}\left(C\left(\tilde{L}_{1}\right) \rightarrow C\left(\tilde{L}_{2}\right)\right. \\
& =\tau^{\mathrm{NEW}}\left(\mathbb{G}_{*}\left(C\left(\tilde{L}_{1}\right)\right) \rightarrow \mathbb{G}_{*}\left(C\left(\tilde{L}_{2}\right)\right)\right) \\
& =p^{!} \tau^{\mathrm{NEW}}\left(C\left(\tilde{K}_{1}\right) \rightarrow C\left(\tilde{K}_{2}\right)\right) .
\end{aligned}
$$

In the general case we choose a common subdivision $\left(L_{3} \rightarrow K_{3}, \mathcal{E}\right)$ with fibre structure $F$. Then:

$$
\begin{aligned}
\Phi\left(\left[L_{1}\right],\left[L_{2}\right]\right) & =\Phi\left(\left[L_{1}\right],\left[L_{3}\right]\right)+\Phi\left(\left[L_{3}\right],\left[L_{2}\right]\right) \\
& =p^{!} \Phi\left(\left[K_{1}\right],\left[K_{3}\right]\right)+p^{!} \Phi\left(\left[K_{3}\right],\left[K_{2}\right]\right) \\
& =p^{!} \Phi\left(\left[K_{1}\right],\left[K_{2}\right]\right) .
\end{aligned}
$$

Thus the Corollary is proved.

The proof of Proposition 9.1 We choose a torsion structure $K \rightarrow B$ representing $[B]$. Then by Theorem 8.5 we may find a fibre bundle torsion structure $(L \rightarrow K, \mathcal{E})$ with the given fibre class. Let $\left(L^{\prime} \rightarrow K^{\prime}, \mathcal{E}^{\prime}\right)$ be another choice of fibre bundle torsion structure. Then by Corollary 9.8 we have

$$
\Phi\left([L],\left[L^{\prime}\right]\right)=p^{!}\left([K],\left[K^{\prime}\right]\right)=p^{!}([B],[B])=0 .
$$

Therefore $[L]$ and $\left[L^{\prime}\right]$ are equivalent.

The proof of Proposition 9.2 Choose representatives $\left(L_{1} \rightarrow K_{1}, \mathcal{E}_{1}\right),\left(L_{2} \rightarrow\right.$ $\left.K_{2}, \mathcal{E}_{2}\right)$ and $\left(L_{3} \rightarrow K_{2}, \mathcal{E}_{3}\right)$ for $\mathfrak{E}_{\left[B_{1}\right]\left[F_{1}\right]}, \mathfrak{E}_{\left[B_{2}\right]\left[F_{1}\right]}$ and $\mathfrak{E}_{\left[B_{2}\right]\left[F_{2}\right]}$ respectively. Then

$$
\begin{aligned}
\Phi\left(\mathfrak{E}_{\left[B_{1}\right]\left[F_{1}\right]}, \mathfrak{E}_{\left[B_{2}\right]\left[F_{2}\right]}\right)= & \Phi\left(\left(L_{1} \rightarrow K_{1}, \mathcal{E}_{1}\right),\left(L_{2} \rightarrow K_{2}, \mathcal{E}_{2}\right)\right) \\
& +\Phi\left(\left(L_{2} \rightarrow K_{2}, \mathcal{E}_{2}\right),\left(L_{3} \rightarrow K_{2}, \mathcal{E}_{3}\right)\right) \\
= & p^{!} \Phi\left(\left[B_{1}\right],\left[B_{2}\right]\right)+\chi(B) \Phi\left(\left[F_{1}\right],\left[F_{2}\right]\right)
\end{aligned}
$$

by Corollary 9.6 and Corollary 9.8 .

The proof of Proposition 9.3 Let $(L \rightarrow K, \mathcal{E})$ be a PFBTS representing $\mathfrak{E}_{[B][F]}$, and recall that its dual torsion structure $\mathcal{D} \mathfrak{E}_{[B][F]}$ is represented by $[\mathcal{D} L]$, where 
$\mathcal{D} \varphi_{0}^{L}: C(\widetilde{\mathcal{D} L}) \cong C(\widetilde{L})^{n+k-*}$, as signed, based complexes (see Definition 5.1). We will first construct a PFBTS representing $\mathfrak{E}_{[\mathcal{D} B][\mathcal{D} F]}$, and then compare these structures.

Note first that $\mathcal{D} L \rightarrow \mathcal{D} K$ is a cellular map and we have a dual PTS for $C(\mathcal{D} K)$. We choose a geometric basis on each subcomplex $p^{-1}(\mathcal{D} \beta) \subset \mathcal{D} L$ such that the composition

$$
C(\widetilde{\mathcal{D} L}) \stackrel{\left(\mathcal{D} \varphi_{0}^{L}\right)^{-1}}{\longrightarrow} C(\widetilde{L})^{n+k-*} \stackrel{{ }^{\theta_{C(\tilde{L})}}}{\longrightarrow} F_{*}^{\text {dual }} C(\widetilde{L})
$$

is the based identity map of signed filtered complexes.

We now have an identification

$$
\mathbb{G}_{*}(C(\widetilde{\mathcal{D} L})) \rightarrow \mathbb{G}_{*}(C(\widetilde{L}))^{k-*}
$$

by taking the associated complex of the above composition, and applying Lemma 12.23 to identify the associated complex of the filtered dual $F_{*}^{\text {dual }} C(\widetilde{L})$ with the $k$-dual $\mathbb{G}_{*}(C(\tilde{L}))^{k-*}$ of the associated complex. We have a chain equivalence

$$
\left(\mathcal{E}^{k-*}\right)^{-1}: \mathbb{G}_{*}(C(\widetilde{\mathcal{D} L})) \rightarrow\left(J^{C(\widetilde{F})} C(\widetilde{K})\right)^{k-*}=J^{C(\widetilde{F}))^{n-*}} C(\widetilde{K})^{k-*}
$$

and this gives an identification

$$
\left(\mathcal{E}^{b_{0}}\right)^{k-*}: C(\tilde{F})^{n-*} \rightarrow C\left(\mathcal{D} p^{-1}\left(\mathcal{D} b_{0}\right)\right) .
$$

However, we may further identify $C\left(\mathcal{D} p^{-1}\left(\mathcal{D} b_{0}\right)\right)$ with $C(\mathcal{D} F)=C\left(\mathcal{D} p^{-1}\left(b_{0}\right)\right)$ using Remark 8.7, since $b_{0}$ is the barycentre of $\mathcal{D} b_{0}$. Then the resulting chain equivalence

$$
\left(\mathcal{E}^{b_{0}}\right)^{k-*}=\mathcal{D} \varphi_{0}^{F}: C(\widetilde{F})^{n-*} \rightarrow C(\widetilde{\mathcal{D F}})
$$

coincides with the original identification of $C(\mathcal{D} F)$ with $C(\widetilde{F})^{n-*}$, given by matching cells to dual cells on $F$. This uses our assumption that the orientations of $F, E$ and $B$ are compatible.

The chain equivalence in (4) doesn't yet give a PFBTS with underlying triangulation $\mathcal{D} L$, since the dual cell complex doesn't have a base-point. Let $p^{\prime}: L^{\prime} \rightarrow K^{\prime}$ be a sub-division of $\mathcal{D} L \rightarrow \mathcal{D} K$ formed by taking the barycentric subdivision over the cell $\mathcal{D} b_{0}$ only. In this new complex we have a base-point $b_{0}$ in $K^{\prime}$. Write $F^{\prime}$ for $\left(p^{\prime}\right)^{-1}\left(b_{0}\right)$.

We choose a PTS on $C\left(\tilde{F}^{\prime}\right)$ such that the subdivision chain equivalence

$$
C\left(\mathcal{D} p^{-1}\left(b_{0}\right)\right) \rightarrow C\left(\widetilde{F}^{\prime}\right)
$$


has trivial absolute torsion, so that $\left[F^{\prime}\right]=[\mathcal{D} F]$. Similarly, we choose a PTS on $C\left(\tilde{K}^{\prime}\right)$ such that the subdivision chain equivalence

$$
C(\widetilde{\mathcal{D K}}) \rightarrow C\left(\tilde{K}^{\prime}\right)
$$

has trivial absolute torsion, so $\left[K^{\prime}\right]=[\mathcal{D} K]$. The subdivision equivalence is the based identity map away from $\mathcal{D} b_{0}$. We now construct a PFBTS $\left(L^{\prime} \rightarrow K^{\prime}, \mathcal{E}^{\prime}\right)$ such that the diagram:

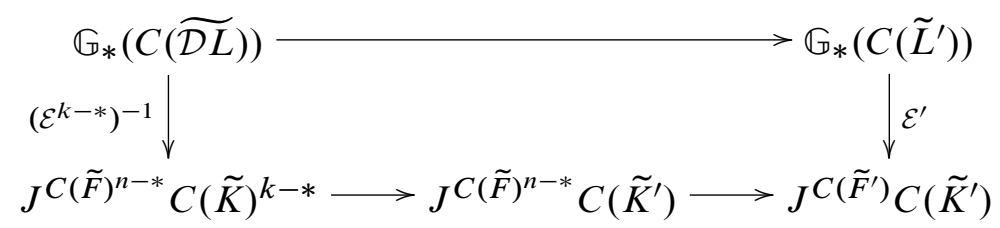

commutes, where the top map is associated to the subdivision equivalence $\mathcal{D} L \rightarrow L^{\prime}$. For cells $\beta \in K^{\prime}$ which correspond to cells in $\mathcal{D} L$ (i.e. those away from $\mathcal{D} b_{0}$ ) we define the map $\mathcal{E}^{\prime \beta}$ to be the composition

$$
C\left(\widetilde{F}_{\beta}^{\prime}\right) \equiv C(\widetilde{\mathcal{D F}} \beta) \stackrel{\left(\left(\mathcal{E}^{\beta}\right)^{k-*}\right)^{-1}}{\longrightarrow} C(\widetilde{F})^{n-*} \stackrel{\mathcal{D} \varphi_{0}^{F}}{\longrightarrow} C(\widetilde{\mathcal{D} F}) \equiv C\left(\widetilde{F}^{\prime}\right) .
$$

Once we have done this, there are unique maps $\mathcal{E}^{\prime \beta}$ for the remaining $\beta$ such that the sum $\mathcal{E}^{\prime}$ is a chain map (existence is a modified form of Theorem 8.5, uniqueness follows by considering boundary pairs). Moreover, the map $\mathcal{E}^{\prime b_{0}}: C\left(\widetilde{F}^{\prime}\right) \rightarrow C\left(\widetilde{F}^{\prime}\right)$ is the identity map, so after appropriate choices of geometric bases for the subcomplexes $F_{\beta}^{\prime}$, we have constructed a PFBTS on $\left(L^{\prime} \rightarrow K^{\prime}, \mathcal{E}^{\prime}\right)$ with fibre class $[\mathcal{D} F]$ and base class $[\mathcal{D} B]$; in other words, this structure represents $\mathfrak{E}_{[\mathcal{D} B][\mathcal{D} F]}$. It remains to show that

$$
\Phi\left(\mathcal{D} \mathfrak{E}_{[B][F]}, \mathfrak{E}_{[\mathcal{D} B][\mathcal{D} F]}\right)=0
$$

which is equivalent to showing that

$$
\Phi\left(\mathcal{D}(L \rightarrow K, \mathcal{E}),\left(L^{\prime} \rightarrow K^{\prime}, \mathcal{E}^{\prime}\right)\right)=0 .
$$

The left, right and bottom maps in diagram (5) all have trivial absolute torsion after applying $i_{*}: K_{1}^{\text {iso }}\left(\mathbb{D A}\left(\mathbb{Z}\left[\pi_{1} E\right]\right)\right) \rightarrow K_{1}\left(\mathbb{Z}\left[\pi_{1} E\right]\right)$, therefore so does the top map. On the other hand, by (4) and Proposition 12.25 we have

$$
\tau^{\mathrm{NEW}}\left(\mathcal{D} \varphi_{0}^{L}: C(\tilde{L})^{n+k-*} \rightarrow C(\widetilde{\mathcal{D} L})\right)=\tau^{\mathrm{NEW}}\left(\theta_{C(\tilde{L})}\right)=0 .
$$


It follows that

$$
\begin{aligned}
\Phi & \left.\mathcal{D}(L \rightarrow K, \mathcal{E}),\left(L^{\prime} \rightarrow K^{\prime}, \mathcal{E}^{\prime}\right)\right) \\
& =\tau^{\mathrm{NEW}}\left(C\left(\widetilde{L}^{\prime}\right) \rightarrow C(\widetilde{L})^{n+k-*}\right) \\
& =\tau^{\mathrm{NEW}}\left(C\left(\widetilde{L}^{\prime}\right) \rightarrow C(\widetilde{\mathcal{D} L})\right)+\tau^{\mathrm{NEW}}\left(C(\widetilde{\mathcal{D} L}) \stackrel{\left(\mathcal{D} \varphi_{0}^{L}\right)^{-1}}{\longrightarrow} C(\widetilde{L})^{n+k-*}\right) \\
& =i_{*} \tau^{\mathrm{NEW}}\left(\mathbb{G}_{*}\left(C\left(\widetilde{L}^{\prime}\right)\right) \rightarrow \mathbb{G}_{*}(C(\widetilde{\mathcal{D} L}))\right) \\
& =0
\end{aligned}
$$

as required.

The proof of Theorem B We can now complete the proof of Theorem B. Let $[B]$ and $[F]$ be torsion structures on $B$ and $F$ respectively. We may assume that $[F] \in$ $\mathcal{S}_{\bullet}(F \stackrel{q}{\rightarrow} E)$ is induced by a PTS on the universal covering of $F$, and therefore $\Phi([\mathcal{D} F],[F])=q_{*}\left(\tau^{\mathrm{NEW}}(F)\right)$ by Theorem 5.2. Then

$$
\begin{aligned}
\tau^{\mathrm{NEW}}(E) & =\Phi\left(\mathcal{D E}_{[B][F]}, \mathfrak{E}_{[B][F]}\right) \\
& =\Phi\left(\mathfrak{E}_{[\mathcal{D} B][\mathcal{D} F]}, \mathfrak{E}_{[B][F]}\right) \\
& =\Phi\left(\mathfrak{E}_{[\mathcal{D} B][F]}, \mathfrak{E}_{[B][F]}\right)+\Phi\left(\mathfrak{E}_{[\mathcal{D} B][\mathcal{D} F]}, \mathfrak{E}_{[\mathcal{D} B][F]}\right) \\
& =p^{!} \Phi([\mathcal{D} B][B])+\chi(B) \Phi([\mathcal{D} F][F]) \\
& =p^{!}\left(\tau^{\mathrm{NEW}}(B)\right)+\chi(B) q_{*}\left(\tau^{\mathrm{NEW}}(F)\right) \in \hat{H}^{n+k}\left(\mathbb{Z} / 2 ; K_{1}\left(\mathbb{Z}\left[\pi_{1} E\right]\right)\right)
\end{aligned}
$$

as required.

\section{The Proof of Theorem A}

Our main result on the multiplicativity of signatures will be deduced from Theorem 3.3 and the following application of Theorem $\mathrm{B}$, in which we compute the reduced absolute torsion $E$. If $\operatorname{dim} B$ is odd, the vanishing of $\operatorname{sign}(E)$ follows immediately (as remarked in Atiyah [2]), so we may assume that $\operatorname{dim} B$ is even.

Theorem 10.1 Let $F \stackrel{q}{\rightarrow} E \stackrel{p}{\rightarrow} B$ be a $P L$ bundle of compatibly oriented closed manifolds. If $\operatorname{dim} B$ is even, then the reduced absolute torsion

$$
\left.\left.\bar{\tau}^{\mathrm{NEW}}(E)=\chi(F) \cdot \bar{\tau}^{\mathrm{NEW}}(B)\right)+\chi(B) \cdot \bar{\tau}^{\mathrm{NEW}}(F)\right) .
$$

Proof The augmentation map induces a direct sum splitting

$$
K_{1}\left(\mathbb{Z}\left[\pi_{1}\left(E, e_{0}\right)\right]\right) \cong K_{1}(\mathbb{Z}) \oplus \tilde{K}_{1}\left(\mathbb{Z}\left[\pi_{1}\left(E, e_{0}\right)\right]\right)
$$


and $\epsilon_{*}$ restricted to the subgroup $K_{1}(\mathbb{Z})$ is the identity map. We will apply the augmentation map $\epsilon_{*}$ to the formula for $\tau^{\mathrm{NEW}}(E)$ given in Theorem B. Note that $\epsilon_{*} \circ q_{*}=\epsilon_{*}$ so the second term

$$
\left.\chi(B) \cdot \epsilon_{*}\left(q_{*} \tau^{\mathrm{NEW}}(F)\right)=\chi(B) \cdot \bar{\tau}^{\mathrm{NEW}}(F)\right)
$$

as required.

To evaluate the first term, we need to compute

$$
\epsilon_{*}\left(p^{!}\left(\tau^{\mathrm{NEW}}(B)\right)\right)=\epsilon_{*}\left(p_{*} p^{!}\left(\tau^{\mathrm{NEW}}(B)\right)\right) .
$$

By Proposition 3.5 we know that $\tau^{\mathrm{NEW}}(B)=\tau\left( \pm h^{2}\right)$ for some $h \in \pi_{1}\left(B, b_{0}\right)$. But the map $\phi: \pi_{1}\left(B, b_{0}\right) \rightarrow K_{1}(\mathbb{Z})$ defined by $\phi(g)=\epsilon_{*}\left(p^{!}(\tau(g))\right)$ is a group homomorphism. Since $K_{1}(\mathbb{Z})=\mathbb{Z} / 2, \phi$ vanishes on squares of group elements in $\pi_{1}\left(B, b_{0}\right)$. Therefore

$$
\epsilon_{*}\left(p^{!}\left(\tau^{\mathrm{NEW}}(B)\right)\right)=\epsilon_{*}\left(p^{!}(\tau( \pm 1))\right) .
$$

However, $\tau( \pm 1)$ lies in the image of the map $i_{*}: K_{1}(\mathbb{Z}) \rightarrow K_{1}\left(\mathbb{Z}\left[\pi_{1}\left(B, b_{0}\right)\right]\right)$ induced by inclusion. By pulling back the bundle $E \stackrel{p}{\rightarrow} B$ over the base point $b_{0} \in B$, and applying the naturality formula for pullbacks (see [12, Corollary 5.3]), we obtain the relation

$$
p^{!}\left(\tau^{\mathrm{NEW}}(B)\right)=i_{*}\left(p_{0}^{*}(\tau( \pm 1))\right)=i_{*}\left(p_{0}^{*}(\tau( \pm 1))\right)
$$

by comparison with the trivial bundle $F \times\left\{b_{0}\right\} \stackrel{p_{0}}{\longrightarrow}\left\{b_{0}\right\}$. But for a trivial bundle $p_{*} \circ p^{!}$is just multiplication by $\chi(F)$, by an easy special case of [13, Theorem 7.1]. Therefore

$$
\epsilon_{*}\left(p^{!}\left(\tau^{\mathrm{NEW}}(B)\right)\right)=\epsilon_{*}\left(p_{*}\left(p^{!}\left(\tau^{\mathrm{NEW}}(B)\right)\right)\right)=\chi(F) \cdot \bar{\tau}^{\mathrm{NEW}}(B)
$$

as required, since $\epsilon_{*} \circ i_{*}$ is the identity map.

Corollary 10.2 If $\operatorname{dim} B$ is even, then $\bar{\tau}^{\mathrm{NEW}}(E)=\bar{\tau}^{\mathrm{NEW}}(F \times B)$.

Proof We apply the product formula, Proposition 6.5, and the formula just proved.

This result says that the reduced absolute torsion of $E$ is the same as that of the total space $F \times B$ for the trivial bundle, provided $\operatorname{dim} B$ is even, We can now conclude that the signature $\operatorname{sign}(E)$ agrees with $\operatorname{sign}(F \times B)=\operatorname{sign}(F) \cdot \operatorname{sign}(B)$ modulo 4 . 
(i) If $\operatorname{dim} B=4 j, \operatorname{dim} F=4 l$, and $\operatorname{dim} E=4(j+l)=4 k$

$$
\begin{array}{rl}
\operatorname{sign}(E)= & 2 \bar{\tau}^{\mathrm{NEW}}(E)+(2 k+1) \chi(E) \\
=2 & 2\left(\bar{\tau}^{\mathrm{NEW}}(B) \chi(F)+\chi(B) \bar{\tau}^{\mathrm{NEW}}(F)\right) \\
& +(2 k+1) \chi(B) \chi(F) \\
\operatorname{sign}(B) \operatorname{sign}(F)= & {\left[2 \bar{\tau}^{\mathrm{NEW}}(b)+(2 j+1) \chi(B)\right]} \\
& {\left[2 \bar{\tau}^{\mathrm{NEW}}(F)+(2 l+1) \chi(F)\right]}
\end{array}
$$

and these agree modulo 4 .

(ii) If $\operatorname{dim} B=4 j+2, \operatorname{dim} F=4 l-2$, and $\operatorname{dim} E=4(j+l)=4 k$

$$
\operatorname{sign}(E)=2\left(\bar{\tau}^{\mathrm{NEW}}(B) \chi(F)+\chi(B) \bar{\tau}^{\mathrm{NEW}}(F)\right)+(2 k+1) \chi(B) \chi(F)
$$

and $\chi(F) \equiv \chi(B) \equiv 0 \bmod 2$, so $\operatorname{sign}(E) \equiv 0 \bmod 4$.

(iii) If $\operatorname{dim} B$ is even, but $\operatorname{dim} F$ is odd, then both sides are zero.

This completes the proof of Theorem A.

\section{Fibrations of PD spaces}

The conjecture of [9] is stated in a more general situation: for fibrations of Poincaré duality spaces. We don't know yet if the signature is multiplicative mod 4 for such fibrations, and hope to return to this topic in a future paper. We will just check that there are no counter-examples arising from finite coverings. A formula for the Whitehead torsions in a fibration of Poincaré duality spaces was given in Pedersen [20].

Recall that C T C Wall constructed examples of finite coverings of oriented Poincaré complexes $X$, with $\pi_{1}(X)=\mathbb{Z} / p, p$ prime and the property that $\operatorname{sign}(\tilde{X}) \neq p \cdot \operatorname{sign}(X)$. On the other hand, the algebraic theory of surgery gives the following congruence.

Lemma 11.1 Let $(f, b): Y \rightarrow X$ be a degree one normal map of oriented, finite Poincaré duality spaces. Then $\operatorname{sign}(Y)-\operatorname{sign}(X) \equiv 0 \bmod 8$.

Proof By [23, page 229] any such degree one normal map has a quadratic signature $\sigma_{*}(f, b)$, with the property that $\operatorname{sign}\left(\sigma_{*}(f, b)\right)=\operatorname{sign}\left(\sigma^{*}(Y)\right)-\operatorname{sign}\left(\sigma^{*}(X)\right)$ and $\operatorname{sign}(X)=\operatorname{sign}\left(\sigma^{*}(X)\right)$ for any Poincaré complex. On the other hand, $\operatorname{sign}\left(\sigma_{*}(f, b)\right) \equiv$ $0 \bmod 8$ since the signature of an even, unimodular symmetric bilinear form over $\mathbb{Z}$ is divisible by 8 (see $[8,3.11])$. 
Corollary 11.2 Let $X^{\prime} \rightarrow X$ be a finite covering of degree $d$ of oriented, finite Poincaré complexes. Then $\operatorname{sign}\left(X^{\prime}\right)-d \cdot \operatorname{sign}(X) \equiv 0 \bmod 8$.

Proof For any finite covering $X^{\prime} \rightarrow X$ of oriented, finite Poincaré duality spaces, we can construct a degree one normal map

$$
(f, b): X^{\prime} \sqcup-(d-1) X \rightarrow X .
$$

Then Lemma 11.1 implies that

$$
\operatorname{sign}\left(X^{\prime}\right)-d \cdot \operatorname{sign}(X)=\operatorname{sign}\left(X^{\prime} \sqcup-(d-1) X\right)-\operatorname{sign}(X) \equiv 0 \bmod 8 .
$$

\section{Filtered chain complexes}

In this section, we give a self-contained treatment of the absolute torsion of signed filtered chain complexes. These results are the algebraic foundation for the torsion calculations in this paper, but this section can be read independently of the previous sections. The main results are the Invariance Theorems Theorem 12.14, Theorem 12.17, generalizing results previously obtained for the reduced torsion by Milnor [17], Maumary [15] and Munkholm [18]. These results express the absolute torsion $\tau^{\mathrm{NEW}}(f) \in K_{1}(\mathbb{A})$ of a filtered chain equivalence $f: C \rightarrow D$ of signed filtered complexes in terms of the filtration quotients.

\subsection{Filtered complexes}

We first need some notation and definitions.

Definition 12.1 Let $\mathbb{A}$ be an additive category.

(i) A $k$-filtered object $F_{*} M$ in $\mathbb{A}$ is an object $M$ in $\mathbb{A}$ together with a direct sum decomposition

$$
M=M_{0} \oplus M_{1} \oplus \cdots \oplus M_{k}
$$

which we regard as a length $k$ filtration

$$
F_{-1} M=0 \subseteq F_{0} M \subseteq F_{1} M \subseteq \cdots \subseteq F_{k} M=M
$$

with

$$
F_{j} M=M_{0} \oplus M_{1} \oplus \cdots \oplus M_{j}(0 \leqslant j \leqslant k) .
$$


(ii) A filtered morphism $f: F_{*} M \rightarrow F_{*} N$ of $k$-filtered objects in $\mathbb{A}$ is a morphism in $\mathbb{A}$ of the type

$$
f=\left(\begin{array}{ccccc}
f_{0} & f_{1} & f_{2} & \ldots & f_{k} \\
0 & f_{0} & f_{1} & \ldots & f_{k-1} \\
0 & 0 & f_{0} & \ldots & f_{k-2} \\
\vdots & \vdots & \vdots & \ddots & \vdots \\
0 & 0 & 0 & \ldots & f_{0}
\end{array}\right): M=\bigoplus_{s=0}^{k} M_{s} \rightarrow N=\bigoplus_{s=0}^{k} N_{s}
$$

so that

$$
f\left(F_{j} M\right) \subseteq F_{j} N(0 \leqslant j \leqslant k) .
$$

The $(u, v)$-component of this upper triangular matrix is a morphism $f_{v-u}: M_{v} \rightarrow$ $N_{u}, 0 \leqslant u \leqslant v \leqslant k$, where $f_{j}: M_{*} \rightarrow N_{*-j}, 0 \leqslant j \leqslant k$, are graded morphisms in $A$.

(iii) A $k$-filtered complex $F_{*} C$ in $\mathbb{A}$ is a finite chain complex $C$ in $\mathbb{A}$ with $k$-filtered objects $C_{r}=\bigoplus_{s=0}^{k} C_{r, s}$ such that the differentials $d: F_{*} C_{r} \rightarrow F_{*} C_{r-1}$ are filtered morphisms. The matrix components of $d$ are maps $d_{j}: C_{r, s} \rightarrow C_{r-1, s-j}$.

(iv) A filtered chain map $f: F_{*} C \rightarrow F_{*} D$ is a chain map $f: C \rightarrow D$ such that $f_{r}: F_{*} C_{r} \rightarrow F_{*} D_{r}$ is a filtered morphism in each degree. The component maps have the form $f_{j}: C_{r, s} \rightarrow D_{r, s-j}$.

(v) A filtered chain homotopy $g: f \simeq f^{\prime}: F_{*} C \rightarrow F_{*} D$ between filtered chain maps $f, f^{\prime}$ is a collection $\left\{g: C_{r} \rightarrow D_{r+1} \mid r \in \mathbb{Z}\right\}$ of morphisms in $\mathbb{A}$ such that

$$
f-f^{\prime}=d g+g d: C_{r} \rightarrow D_{r}
$$

and

$$
g=\left(\begin{array}{ccccc}
g_{0} & g_{1} & g_{2} & \ldots & g_{k} \\
g_{-1} & g_{0} & g_{1} & \ldots & g_{k-1} \\
0 & g_{-1} & g_{0} & \ldots & g_{k-2} \\
\vdots & \vdots & \vdots & \ddots & \vdots \\
0 & 0 & 0 & \ldots & g_{0}
\end{array}\right)
$$

has component maps

$$
g_{j}: C_{r, s} \rightarrow D_{r+1, s-j}(-1 \leqslant j \leqslant k) .
$$

(vi) A filtered contraction of a $k$-filtered complex $F_{*} C$ in $\mathbb{A}$ is a filtered chain homotopy

$$
\Gamma: 1 \simeq 0: F_{*} C \rightarrow F_{*} C .
$$


(vii) The filtered mapping cone of a filtered chain map $f: C \rightarrow D$ of $k$-filtered complexes is the $(k+1)$-filtered complex $F_{*} \mathcal{C}^{\text {fil }}(f)$ with

$$
\mathcal{C}^{\text {fil }}(f)_{r, s}=D_{r, s} \oplus C_{r-1, s-1}(0 \leqslant s \leqslant k+1)
$$

and the differential $d^{\mathcal{C}^{\text {fil }}(f)}: \mathcal{C}^{\text {fil }}(f)_{r} \rightarrow \mathcal{C}^{\text {fil }}(f)_{r-1}$ is in upper triangular block form with $2 \times 2$ block entries $(0 \leqslant j \leqslant k+1)$ :

$$
d_{j}^{\mathcal{C}^{\mathrm{fil}}(f)}=\left(\begin{array}{cc}
d_{j}^{D}(-)^{r-1} f_{j-1} \\
0 & d_{j}^{C}
\end{array}\right): D_{r, s} \oplus C_{r-1, s-1} \rightarrow D_{r-1, s-j} \oplus C_{r-2, s-j-1} .
$$

The rearrangement map

$$
\mathcal{C}^{\mathrm{fil}}(f)_{r}=\sum_{s=0}^{k}\left(D_{r, s} \oplus C_{r-1, s-1}\right) \stackrel{\cong}{\rightrightarrows} \sum_{s=0}^{k} D_{r, s} \oplus \sum_{s=1}^{k} C_{r-1, s-1}=\mathcal{C}(f)_{r}
$$

defines an isomorphism of unfiltered chain complexes $\rho: \mathcal{C}^{\text {fil }}(f) \stackrel{\cong}{\longrightarrow} \mathcal{C}(f)$.

Theorem 12.2 A filtered chain map $f: F_{*} C \rightarrow F_{*} D$ of $k$-filtered chain complexes is a filtered chain equivalence if and only if the filtered mapping cone $F_{*} \mathcal{C}^{\mathrm{fil}}(f)$ is filtered contractible.

Proof By definition, $f$ is a filtered chain equivalence if and only if there exist a filtered chain map $g: F_{*} D \rightarrow F_{*} C$ and filtered chain homotopies

$$
\Gamma: 1 \simeq g f: F_{*} C \rightarrow F_{*} C, \Delta: 1 \simeq f g: F_{*} D \rightarrow F_{*} D .
$$

A filtered contraction $e: 1 \simeq 0: F_{*} \mathcal{C}^{\text {fil }}(f) \rightarrow F_{*} \mathcal{C}^{\text {fil }}(f)$ is of the type

$$
e=\left(\underset{(-)^{r} g}{\Delta} \stackrel{h}{\Gamma}\right): \mathcal{C}^{\mathrm{fil}}(f)_{r} \cong D_{r} \oplus C_{r-1} \rightarrow \mathcal{C}^{\text {fil }}(f)_{r+1} \cong D_{r+1} \oplus C_{r}
$$

with $g: F_{*} D \rightarrow F_{*} C$ a filtered chain map and $\Gamma: 1 \simeq g f, \Delta: 1 \simeq f g$ filtered chain homotopies, so that $f$ is a filtered chain equivalence.

Conversely, suppose that $f$ is a filtered chain equivalence. The morphisms defined by

$$
e^{\prime}=\left(\begin{array}{cc}
\Delta & \Delta)^{r} g \\
\Gamma
\end{array}\right): \mathcal{C}^{\text {fil }}(f)_{r} \cong D_{r} \oplus C_{r-1} \rightarrow \mathcal{C}^{\text {fil }}(f)_{r+1} \cong D_{r+1} \oplus C_{r}
$$

are such that the morphisms

$$
d e^{\prime}+e^{\prime} d=\left(\begin{array}{c}
1(-)^{r}(f \Gamma-\Delta f) \\
0 \\
1
\end{array}\right): \mathcal{C}^{\text {fil }}(f)_{r} \cong D_{r} \oplus C_{r-1} \rightarrow \mathcal{C}^{\text {fil }}(f)_{r} \cong D_{r} \oplus C_{r-1}
$$

are automorphisms. The morphisms

$$
e=\left(d e^{\prime}+e^{\prime} d\right)^{-1} e^{\prime}: \mathcal{C}^{\mathrm{fil}}(f)_{r} \cong D_{r} \oplus C_{r-1} \rightarrow \mathcal{C}^{\mathrm{fil}}(f)_{r+1} \cong D_{r+1} \oplus C_{r}
$$

define a filtered contraction $e: 1 \simeq 0: F_{*} \mathcal{C}^{\text {fil }}(f) \rightarrow F_{*} \mathcal{C}^{\text {fil }}(f)$. 


\subsection{The associated complex}

We now describe how to pass from a filtered complex to its associated graded complex. The differentials $d: C_{r} \rightarrow C_{r-1}$ of a $k$-filtered complex $F_{*} C$ satisfy the matrix identities

$$
d^{2}=0: C_{r} \rightarrow C_{r-2} \text {. }
$$

By carrying out the multiplication $d^{2}$ as a product of upper triangular matrices, we obtain a number of recursion formulas among the component maps $d_{j}: C_{r, s} \rightarrow C_{r-1, s-j}$. In particular, we have the formulas

$$
\begin{aligned}
&\left(d_{0}\right)^{2}=0: C_{r, s} \rightarrow C_{r-2, s}, \\
& d_{0} d_{1}+d_{1} d_{0}=0: C_{r, s} \rightarrow C_{r-2, s-1}, \\
&\left(d_{1}\right)^{2}+d_{0} d_{2}+d_{2} d_{0}=0: C_{r, s} \rightarrow C_{r-2, s-2} .
\end{aligned}
$$

Definition 12.3 The associated complex of a $k$-filtered complex $F_{*} C$ in $\mathbb{A}$ is the $k$-dimensional chain complex in the derived category $\mathbb{D}(\mathbb{A})$

$$
\mathbb{G}_{*}(C): G_{k}(C) \rightarrow \cdots \rightarrow G_{r+1}(C) \stackrel{d_{*}}{\rightarrow} G_{r}(C) \stackrel{d_{*}}{\longrightarrow} \cdots \rightarrow G_{0}(C)
$$

with differential

$$
d_{*}=(-)^{s} d_{1}: G_{r}(C)_{s}=C_{r+s, r} \rightarrow G_{r-1}(C)_{s}=C_{r+s-1, r-1} .
$$

The individual terms $G_{r}(C)$ are objects in $\mathbb{D}(\mathbb{A})$, with 'internal' differential

$$
d_{G_{r}(C)}=d_{0}: G_{r}(C)_{s}=C_{r+s, r} \rightarrow G_{r}(C)_{s-1}=C_{r+s-1, r} .
$$

Example 12.4 (Tensor products) Let $C$ and $D$ be chain complexes in $\mathbb{A}(R)$ and $\mathbb{A}(S)$ respectively, where $R$ and $S$ are rings. If $\operatorname{dim} C=k$, then the tensor product complex $C \otimes D($ over $\mathbb{Z})$ admits a $k$-filtered structure with $(C \otimes D)_{r, s}=C_{s} \otimes D_{r-s}$ for $0 \leqslant s \leqslant k$. The filtered differential is defined by

$$
\begin{aligned}
& d_{0}=1 \otimes d^{D}: C_{s} \otimes D_{r-s} \rightarrow C_{s} \otimes D_{r-s-1} \\
& d_{1}=(-)^{r-s} d^{C} \otimes 1: C_{s-1} \otimes D_{r-s} \rightarrow C_{s-1} \otimes D_{r-s}
\end{aligned}
$$

and $d_{j}=0$ for $j \geqslant 2$. The associated complex has $G_{r}(C \otimes D)_{s}=C_{r} \otimes D_{s}$ and differential $d_{*}=d^{C} \otimes 1: C_{r} \otimes D_{s} \rightarrow C_{r-1} \otimes D_{s}$.

When we work with the associated complex, it is useful to translate the maps $d_{j}: C_{r, s} \rightarrow$ $C_{r-1, s-j}$ into the new notation, so that

$$
d_{j}: G_{r}(C)_{s} \rightarrow G_{r-j}(C)_{s+j-1} .
$$


The relation $d_{0} d_{1}+d_{1} d_{0}=0$ then implies that $d_{*}: G_{r}(C) \rightarrow G_{r-1}(C)$ is a chain map. The relation $\left(d_{1}\right)^{2}+d_{0} d_{2}+d_{2} d_{0}=0$ shows that $\left(d_{1}\right)^{2}$ is chain homotopic to zero, and hence $\left(d_{*}\right)^{2}=0$ in the derived category.

\section{Proposition 12.5}

(i) A filtered chain map $f: F_{*} C \rightarrow F_{*} D$ induces a chain map in $\mathbb{D}(\mathbb{A})$

$$
\mathbb{G}_{*}(f): \mathbb{G}_{*}(C) \rightarrow \mathbb{G}_{*}(D) .
$$

(ii) A filtered chain homotopy $g: f \simeq f^{\prime}: F_{*} C \rightarrow F_{*} D$ induces a chain homotopy in $\mathbb{D}(\mathbb{A})$

$$
\mathbb{G}_{*}(g): \mathbb{G}_{*}(f) \simeq \mathbb{G}_{*}\left(f^{\prime}\right): \mathbb{G}_{*}(C) \rightarrow \mathbb{G}_{*}(D) .
$$

(iii) A filtered chain equivalence $f: F_{*} C \rightarrow F_{*} D$ induces a chain equivalence $\mathbb{G}_{*}(f): \mathbb{G}_{*}(C) \rightarrow \mathbb{G}_{*}(D)$ in $\mathbb{D}(\mathbb{A})$.

(iv) A filtered chain contraction $\Gamma: 1 \simeq 0: F_{*} C \rightarrow F_{*} D$ induces a chain equivalence $\mathbb{G}_{*}(\Gamma): 1 \simeq 0: \mathbb{G}_{*}(C) \rightarrow \mathbb{G}_{*}(D)$ in $\mathbb{D}(\mathbb{A})$.

\section{Proof}

(i) For a filtered chain map $f: F_{*} C \rightarrow F_{*} D$ the identities

$$
d f=f d: C_{r} \rightarrow D_{r}
$$

expressed in upper triangular matrix form lead to relations involving the component maps $f_{i}$ and $d_{j}$. In particular we obtain $d_{0} f_{0}=f_{0} d_{0}: C_{r, s} \rightarrow D_{r-1, s}$ and the map $G_{r}(f)=f_{0}: G_{r}(C) \rightarrow G_{r}(D)$ is a chain map. The relation $d_{1} f_{0}-f_{0} d_{1}=f_{1} d_{0}-d_{0} f_{1}$ shows that $(-)^{s} f_{1}: G_{r}(C)_{s} \rightarrow G_{r-1}(D)_{s+1}$ gives a chain homotopy $d_{*} f_{0}-f_{0} d_{*} \simeq 0$, and so $f_{0}$ gives a chain map $\mathbb{G}_{*}(f): \mathbb{G}_{*}(C) \rightarrow \mathbb{G}_{*}(D)$ in $\mathbb{D}(\mathbb{A})$.

(ii) For a filtered chain homotopy $g: f \simeq f^{\prime}: F_{*} C \rightarrow F_{*} D$ the identities

$$
f-f^{\prime}=d g+g d: C_{r} \rightarrow D_{r}
$$

expressed in upper triangular matrix form again lead to various relations. In particular

$$
\begin{aligned}
d_{0} g_{-1}+g_{-1} d_{0} & =0: C_{r, s} \rightarrow D_{r, s+1}, \\
f_{0}-f_{0}^{\prime} & =d_{0} g_{0}+g_{0} d_{0}+d_{1} g_{-1}+g_{-1} d_{1}: C_{r, s} \rightarrow D_{r, s} .
\end{aligned}
$$

In the $\mathbb{G}_{*}$-notation, we have maps

$$
g_{j}: G_{r}(C)_{s} \rightarrow G_{r-j}(D)_{s+j+1} .
$$

Since $g_{0}: G_{r}(C)_{s} \rightarrow G_{r}(D)_{s+1}$ gives a chain null-homotopy $d_{0} g_{0}+g_{0} d_{0} \simeq 0: G_{r}(C) \rightarrow$ $G_{r}(D)$, the map

$$
\mathbb{G}_{*}(g)=(-)^{s} g_{-1}: G_{r}(C)_{s} \rightarrow G_{r+1}(D)_{s}
$$


gives a null-homotopy $f_{0}-f_{0}^{\prime} \simeq 0$ in the derived category $\mathbb{D}(\mathbb{A})$. Parts (iii) and (iv) follow immediately from parts (i) and (ii).

\section{Theorem 12.6}

(i) A $k$-filtered complex $F_{*} C$ in $\mathbb{A}$ is filtered contractible if and only if the associated complex $\mathbb{G}_{*}(C)$ is contractible in $\mathbb{D}(\mathbb{A})$.

(ii) A filtered chain map $f: F_{*} C \rightarrow F_{*} D$ of $k$-filtered chain complexes is a filtered chain equivalence if and only if the associated chain map $\mathbb{G}_{*}(f): \mathbb{G}_{*}(C) \rightarrow$ $\mathbb{G}_{*}(D)$ is a chain equivalence in $\mathbb{D}(\mathbb{A})$.

Proof (i) A filtered contraction $\Gamma: 1 \simeq 0: F_{*} C \rightarrow F_{*} C$ induces a contraction $\mathbb{G}_{*}(\Gamma): 1 \simeq 0: \mathbb{G}_{*}(C) \rightarrow \mathbb{G}_{*}(C)$ by Proposition 12.5 (iv).

Conversely, suppose given a contraction of $\mathbb{G}_{*}(C)$ in $\mathbb{D}(\mathbb{A})$

$$
e: 1 \simeq 0: \mathbb{G}_{*}(C) \rightarrow \mathbb{G}_{*}(C),
$$

as represented by chain maps $e:\left(G_{r}(C), d_{0}\right) \rightarrow\left(G_{r+1}(C), d_{0}\right)$, for which there exist chain homotopies

$$
h: 1 \simeq d_{*} e+e d_{*}: G_{r}(C) \rightarrow G_{r}(C)
$$

with

$$
(-)^{s}\left(d_{1} e+e d_{1}\right)+d_{0} h+h d_{0}=1: G_{r}(C)_{s}=C_{r+s, r} \rightarrow G_{r}(C)_{s}=C_{r+s, r} .
$$

In terms of the filtration on $C$, the maps $h: G_{r}(C)_{s} \rightarrow G_{r}(C)_{s+1}$ and $e: G_{r}(C)_{s} \rightarrow$ $G_{r+1}(C)_{s}$ give maps $h_{s}: C_{r, s} \rightarrow C_{r+1, s}$ and $e_{r, s}: C_{r, s} \rightarrow C_{r+1, s+1}$. The relation given by $h: 1 \simeq d_{*} e+e d_{*}$ gives

$$
(-)^{r+s}\left(d_{1} e+e d_{1}\right)+d_{0} h+h d_{0}=1: C_{r, s} \rightarrow C_{r, s} .
$$

Let $\widehat{e}_{r, s}=(-)^{r-1} e$, and define morphisms

$$
\beta=\left(\begin{array}{ccccc}
h & 0 & 0 & \ldots & 0 \\
\hat{e} & h & 0 & \ldots & 0 \\
0 & \widehat{e} & h & \ldots & 0 \\
\vdots & \vdots & \vdots & \ddots & \\
0 & 0 & 0 & \ldots & h
\end{array}\right): C_{r} \rightarrow C_{r+1}
$$

The morphisms $\alpha: C_{r} \rightarrow C_{r}$ defined by

$$
\alpha=d \beta+\beta d: C_{r} \rightarrow C_{r}
$$


are in upper triangular form, with the identity on the main diagonal. For the components in the $(s+1, s)$ positions, note that we get $(-)^{r-1} d_{0} e+(-)^{r} e d_{0}=0$ since $e$ a chain map. In the diagonal $(s, s)$ positions we get the sum of composites

$$
\begin{array}{ll}
C_{r, s} \stackrel{(-)^{s} d_{1}}{\longrightarrow} C_{r-1, s-1} \stackrel{(-)^{r} e}{\longrightarrow} C_{r, s} & C_{r, s} \stackrel{(-)^{r-1} e}{\longrightarrow} C_{r+1, s+1} \stackrel{(-)^{s+1} d_{1}}{\longrightarrow} C_{r, s} \\
C_{r, s} \stackrel{d_{0}}{\longrightarrow} C_{r-1, s} \stackrel{h}{\rightarrow} C_{r, s} & C_{r, s} \stackrel{h}{\rightarrow} C_{r+1, s} \stackrel{d_{0}}{\longrightarrow} C_{r, s}
\end{array}
$$

which equals $(-)^{r+s}\left(d_{1} e+e d_{1}\right)+d_{0} h+h d_{0}=1$ by the relation above.

Each $\alpha: C_{r} \rightarrow C_{r}$ is an automorphism, with

$$
\begin{aligned}
d \alpha & =d \beta d=\alpha d: C_{r} \rightarrow C_{r-1}, \\
\alpha^{-1} d & =d \alpha^{-1}: C_{r} \rightarrow C_{r-1} .
\end{aligned}
$$

The morphisms

$$
\Gamma=\beta \alpha^{-1}: C_{r} \rightarrow C_{r+1}
$$

are such that

$$
d \Gamma+\Gamma d=(d \beta+\beta d) \alpha^{-1}=1: C_{r} \rightarrow C_{r},
$$

and define a filtered contraction of $F_{*} C$

$$
\Gamma: 1 \simeq 0: C \rightarrow C .
$$

(ii) By Theorem $12.2 f$ is a filtered chain equivalence if and only if $F_{*} \mathcal{C}^{\text {fil }}(f)$ is filtered contractible. By (i) $F_{*} \mathcal{C}^{\text {fil }}(f)$ is filtered contractible if and only if $\mathbb{G}_{*}\left(\mathcal{C}^{\text {fil }}(f)\right)=$ $\mathcal{C}\left(G_{*}(f)\right)$ is chain contractible.

\subsection{Splitting and Folding}

In an additive category $\mathbb{A}$ we don't necessarily have kernels and cokernels, but we can define split exact sequences. A direct sum system

$$
A \underset{\Gamma}{\stackrel{f}{\rightleftarrows}} B \underset{\Delta}{\stackrel{g}{\rightleftarrows}} C
$$

is a collection of morphisms in $\mathbb{A}$ such that

$$
(\Delta f): C \oplus A \rightarrow B, \quad\left(\begin{array}{l}
g \\
\Gamma
\end{array}\right): B \rightarrow C \oplus A
$$

are inverse isomorphisms in $\mathbb{A}$. Then we say that a morphism $f: A \rightarrow B$ in $\mathbb{A}$ is a split injection (respectively $g: B \rightarrow C$ is a split surjection) if the morphism extends to a direct sum system. Then

$$
0 \rightarrow A \stackrel{f}{\rightarrow} B \stackrel{g}{\rightarrow} C \rightarrow 0
$$


is a short exact sequence in $\mathbb{A}$ if $f$ and $g$ can be extended to a direct sum system. A short exact sequence of chain complexes in $\mathbb{A}$ is one which is short exact in each degree.

There is a useful criterion for the existence of a split injection in the derived category $\mathbb{D}(\mathbb{A})$, expressed in terms of any representative chain map $f$ for a given morphism.

Proposition 12.7 A morphism $f: C \rightarrow D$ in $\mathbb{D}(\mathbb{A})$ is a split injection if and only if there exists a chain map $\Gamma: D \rightarrow C$ and a chain homotopy $h: \Gamma f \simeq 1: C \rightarrow C$. If $(\Gamma, h)$ exists, then there is a direct sum system

$$
C \underset{\Gamma}{\stackrel{f}{\rightleftarrows}} D \underset{\Delta}{\stackrel{g}{\rightleftarrows}} \mathcal{C}(f)
$$

in $\mathbb{D}(\mathbb{A})$, with $g=\left(\begin{array}{l}1 \\ 0\end{array}\right): D_{r} \rightarrow \mathcal{C}(f)_{r}=D_{r} \oplus C_{r-1}$ and

$$
\Delta=\left(1-f \Gamma(-)^{r+1} f h\right): \mathcal{C}(f)_{r}=D_{r} \oplus C_{r-1} \rightarrow D_{r} .
$$

Proof Assuming there exist such $\Gamma, h$ we define a direct sum system in $\mathbb{D}(\mathbb{A})$ by the given formulas. The chain maps

$$
(\Delta f): \mathcal{C}(f) \oplus C \rightarrow D,\left(\begin{array}{l}
g \\
\Gamma
\end{array}\right): D \rightarrow \mathcal{C}(f) \oplus C
$$

are inverse chain equivalences, and the morphisms

$$
e=\left(\begin{array}{ccc}
0 & 0 & 0 \\
(-)^{r} \Gamma & -h & (-)^{r+1} \\
h \Gamma & (-)^{r+1} h^{2} & h
\end{array}\right): D_{r} \oplus C_{r-1} \oplus C_{r} \rightarrow D_{r+1} \oplus C_{r} \oplus C_{r+1}
$$

define a chain homotopy

$$
e:\left(\begin{array}{l}
g \\
\Gamma
\end{array}\right)(\Delta f) \simeq 1: \mathcal{C}(f) \oplus C \rightarrow \mathcal{C}(f) \oplus C .
$$

The converse is clear.

Corollary 12.8 Let $f: C \rightarrow D$ be a split injection in $\mathbb{S} \mathbb{D}(\mathbb{A})$. Then

$$
i_{*} \tau^{\mathrm{NEW}}\left(\begin{array}{l}
g \\
\Gamma
\end{array}\right)=i_{*} \tau^{\mathrm{NEW}}(\Delta f)=0 \in K_{1}^{\mathrm{iso}}(\mathbb{A}) .
$$

Proof This follows immediately from the last result and the formula in [11, Proposition 13.6], since $\tau^{\mathrm{NEW}}(f)=\tau^{\mathrm{NEW}}(\mathcal{C}(f))$.

We will need a variant of the folding construction used by Whitehead [29] to define the torsion of a contractible complex. Let

$$
C: C_{k} \stackrel{d}{\rightarrow} C_{k-1} \stackrel{d}{\rightarrow} C_{k-2} \rightarrow \cdots \rightarrow C_{0}
$$


be a $k$-dimensional chain complex in $\mathbb{A}$, such that the boundary map $d: C_{k} \rightarrow C_{k-1}$ extends to a direct sum system

$$
C_{k} \underset{\Gamma}{\stackrel{d}{\rightleftarrows}} C_{k-1} \underset{\Delta}{\stackrel{g}{\rightleftarrows}} C_{k-1}^{\prime}
$$

We use this direct sum system to define the $(k-1)$-dimensional chain complex

$$
C^{\prime}: C_{k-1}^{\prime} \stackrel{d \Delta}{\longrightarrow} C_{k-2} \stackrel{d}{\rightarrow} C_{k-3} \rightarrow \cdots \rightarrow C_{0}
$$

in $\mathbb{A}$, called the abelian $k$-folding of $C$. An elementary chain complex in $\mathbb{A}$ is a contractible complex with non-zero chain groups only in two adjacent degrees.

Proposition 12.9 Let $C^{\prime}$ be the abelian $k$-folding of $C$, and let $E$ be the elementary chain complex with $C_{k} \stackrel{1}{\rightarrow} C_{k}$ in adjacent degrees $(k, k-1)$. Then there exists an isomorphism $\left(\begin{array}{l}i \\ j\end{array}\right): C \cong C^{\prime} \oplus E$ with isomorphism torsion

$$
\left.\tau^{\text {iso }}\left(\left(\begin{array}{l}
i \\
j
\end{array}\right)\right)=(-)^{k-1} \tau^{\text {iso }}\left(\left(\begin{array}{l}
g \\
\Gamma
\end{array}\right)\right): C_{k-1} \rightarrow C_{k-1}^{\prime} \oplus C_{k}\right) \in K_{1}^{\text {iso }}(\mathbb{A}) .
$$

Proof The chain isomorphism $\left(\begin{array}{l}i \\ j\end{array}\right): C \rightarrow C^{\prime} \oplus E$ is defined by the diagram

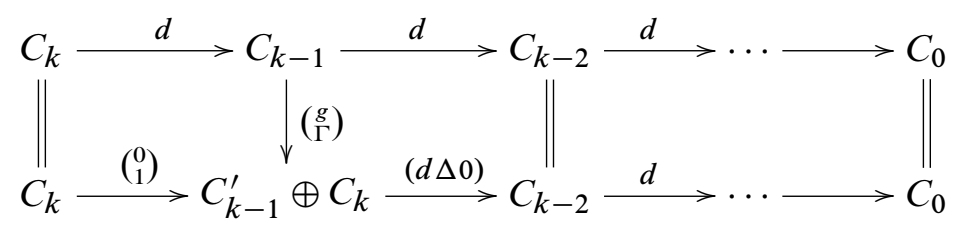

and the relations $g d=0, \Gamma d=1$.

\subsection{The Invariance Theorem for filtered contractible complexes}

We will first establish the sign conventions for filtered complexes.

Definition 12.10 A $k$-filtered signed complex $\left(F_{*} C, \eta_{F_{*} C}\right)$ in $\mathbb{A}$ is a $k$-filtered complex $C$ together with signs

$$
\begin{aligned}
& \eta_{\mathbb{G}_{*}(C)} \in \operatorname{im}\left(\epsilon: K_{0}(\mathbb{S} \mathbb{D}(\mathbb{A})) \otimes K_{0}(\mathbb{S} \mathbb{D}(\mathbb{A})) \rightarrow K_{1}^{\text {iso }}(\mathbb{S} \mathbb{D}(\mathbb{A}))\right) \\
& \eta_{G_{j}(C)} \in \operatorname{im}\left(\epsilon: K_{0}(\mathbb{A}) \otimes K_{0}(\mathbb{A}) \rightarrow K_{1}^{\text {iso }}(\mathbb{A})\right)(0 \leqslant j \leqslant k) .
\end{aligned}
$$

The sign of $F_{*} C$ and the sign of $C$ (as an unfiltered complex) are set to be

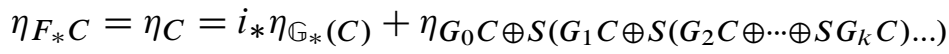

$$
\begin{aligned}
& \in \operatorname{im}\left(\epsilon: K_{0}(\mathbb{A}) \otimes K_{0}(\mathbb{A}) \rightarrow K_{1}^{\text {iso }}(\mathbb{A})\right) .
\end{aligned}
$$


The associated chain complex $\left(\mathbb{G}_{*}(C), \eta_{\mathbb{G}_{*}}(C)\right)$ is a $k$-dimensional chain complex in $\operatorname{SD}(A)$. We will usually denote this signed complex in $\mathbb{D}(\mathbb{A})$ just by $\mathbb{G}_{*}(C)$.

The sign conventions have been chosen so that for a filtered contractible $k$-filtered signed complex $\left(F_{*} C, \eta_{F_{*} C}\right)$ in $\mathbb{A}$

$$
\tau^{\mathrm{NEW}}\left(C, \eta_{C}\right)=\tau^{\mathrm{NEW}}\left(F_{*} C, \eta_{F_{*} C}\right) \in K_{1}^{\mathrm{iso}}(\mathbb{A}) .
$$

The underlying (unfiltered) complex of a filtered complex has a useful iterated mapping cone description. Given a $k$-filtered chain complex $F_{*} C$ in $\mathbb{A}$, we define chain complexes $T_{\ell, r}(C)=S^{-r}\left(F_{\ell} C / F_{r-1} C\right)$ with chain groups

$$
T_{\ell, r}(C)_{s}=\left(F_{\ell} C / F_{r-1} C\right)_{r+s}=C_{r+s, r} \oplus C_{r+s, r+1} \oplus \cdots \oplus C_{r+s, \ell}
$$

for $1 \leqslant r \leqslant \ell \leqslant k$. The differential on $T_{\ell, r}(C)$ is the one induced by $d: C \rightarrow C$, and the sign

$$
\eta_{T_{\ell, r}(C)}=\eta_{G_{r} C \oplus S\left(G_{r+1} C \oplus S\left(G_{r+2} C \oplus \cdots \oplus S G_{\ell} C\right) \ldots\right) .}
$$

Note that the definition $G_{r}(C)_{s}=C_{r+s, r}$ gives the formula

$$
T_{\ell, r}(C)_{s}=G_{r}(C)_{s} \oplus G_{r+1}(C)_{s-1} \oplus \cdots \oplus G_{\ell}(C)_{r+s-\ell} .
$$

We use this expression to define maps

$$
\partial_{\ell, r}: T_{\ell, r}(C)_{s} \rightarrow G_{r-1}(C)_{s} \quad(1 \leqslant j \leqslant k)
$$

by the row matrix

$$
\partial_{\ell, r}=(-)^{s}\left(d_{1} d_{2} \cdots d_{\ell-r+1}\right)
$$

where $d_{j}: G_{r}(C)_{s} \rightarrow G_{r-j}(C)_{s+j-1}$ are the entries in the matrix expression for $d$. If $\ell=k$ we will set $\partial_{r}:=\partial_{k, r}$ to simplify the notation. We remark that $G_{r}(C)=T_{r, r}(C)$ and $\partial_{r, r}=d_{*}: G_{r}(C) \rightarrow G_{r-1}(C)$.

Proposition 12.11 Let $\left(F_{*} C, \eta_{F_{*} C}\right)$ be a $k$-filtered signed complex in $\mathbb{A}$.

(i) The maps $\partial_{\ell, r}: T_{\ell, r}(C) \rightarrow G_{r-1}(C)$ are chain maps.

(ii) $T_{\ell, r-1}(C)=\mathcal{C}\left(T_{\ell, r}(C) \stackrel{\partial_{\ell, r}}{\longrightarrow} G_{r-1}(C)\right)$, for $1 \leqslant r \leqslant \ell \leqslant k$, as signed complexes.

(iii) There is a short exact sequence

$$
T_{\ell_{1}, r}(C) \rightarrow T_{\ell_{2}, r}(C) \rightarrow S^{\ell_{1}-r+1} T_{\ell_{2}, \ell_{1}+1}(C)
$$

in $\operatorname{SDD}(\mathbb{A})$, for $1 \leqslant r \leqslant \ell_{1}<\ell_{2} \leqslant k$.

(iv) The signed complex $\left(C, \eta_{C}-i_{*} \eta_{\mathbb{G}_{*}(C)}\right)=T_{k, 0}(C)$ as an object in $\mathbb{S} \mathbb{D}(\mathbb{A})$. 
Proof The claim that $\partial_{\ell, r}$ is a chain map follows immediately from the relation $d^{2}=0$. Part (ii) follows directly from the definition, and part (iii) can be checked inductively. Note that, according to our conventions, the sign term

$$
\eta_{C}-i_{*} \eta_{\mathbb{G}_{*}(C)}=\eta_{G_{0} C \oplus S\left(G_{1} C \oplus S\left(G_{2} C \oplus \cdots \oplus S G_{k} C\right) \ldots\right)}
$$

is just the sign of the iterated mapping cone structure on $T_{k, 0}(C)$.

Definition 12.12 Let $\left(F_{*} C, \eta_{F_{*} C}\right)$ be a $k$-filtered signed complex in $\mathbb{A}$. The $(k-1)-$ amalgamation of $\left(F_{*} C, \eta_{F_{*} C}\right)$ is the $(k-1)$-filtered signed complex $\left(F_{*} C^{\prime}, \eta_{F_{*}} C^{\prime}\right)$ in $A$ with filtration summands

$$
C_{r, s}^{\prime}=C_{r, s}, \text { if } 0 \leqslant s<k-1 \text { and } C_{r, k-1}^{\prime}=C_{r, k-1} \oplus C_{r, k}
$$

in each degree $r$. The differentials $d_{j}^{\prime}=d_{j}$ except in filtration degree $k-1$, where

$$
d_{j}^{\prime}=\left(\begin{array}{cc}
d_{j} & d_{j+1} \\
0 & d_{j}
\end{array}\right): C_{r, k-1} \oplus C_{r, k} \rightarrow C_{r-1, k-1-j} \oplus C_{r-1, k-j}
$$

and

$$
G_{r}\left(C^{\prime}\right)= \begin{cases}G_{r}(C) & \text { if } 0 \leqslant r \leqslant k-2 \\ \mathcal{C}\left(G_{k}(C) \stackrel{\partial_{k}}{\rightarrow} G_{k-1}(C)\right) & \text { if } r=k-1 .\end{cases}
$$

where $\partial_{k}:=\partial_{k, k}$. The signs are given by the formulas

$$
\eta_{\mathbb{G}_{*}\left(C^{\prime}\right)}=\eta_{\mathbb{G}_{*}(C)}, \eta_{G_{j}\left(C^{\prime}\right)}= \begin{cases}\eta_{G_{j}(C)} & \text { if } 0 \leqslant j \leqslant k-2 \\ \eta_{G_{k-1}(C) \oplus S G_{k}(C)} & \text { if } j=k-1 .\end{cases}
$$

Lemma 12.13 $G_{k-1}\left(C^{\prime}\right)=\mathcal{C}\left(G_{k}(C) \stackrel{\partial_{k}}{\longrightarrow} G_{k-1}(C)\right)$ as objects in $\mathbb{S} \mathbb{D}(\mathbb{A})$.

Proof The associated $(k-1)$-dimensional chain complex $\mathbb{G}_{*}\left(C^{\prime}\right)$ in $\mathbb{S} \mathbb{D}(\mathbb{A})$ has the differential $d_{*}^{\prime}: G_{r}\left(C^{\prime}\right) \rightarrow G_{r-1}\left(C^{\prime}\right)$ given by

$$
d_{\mathbb{G}_{*}\left(C^{\prime}\right)}= \begin{cases}d_{*}: G_{j}(C) \rightarrow G_{j-1}(C) & \text { if } 0 \leqslant j \leqslant k-2 \\ \partial_{k-1}: \mathcal{C}\left(G_{k}(C) \stackrel{\partial_{k}}{\longrightarrow} G_{k-1}(C)\right) \rightarrow G_{k-2}(C) & \text { if } j=k-1 .\end{cases}
$$

Since $G_{k-1}\left(C^{\prime}\right)=G_{k-1}(C) \oplus S G_{k}(C)$, the signs on $G_{k-1}\left(C^{\prime}\right)$ and $\mathcal{C}\left(\partial_{k}\right)$ agree.

Note that $\eta_{G_{0} C^{\prime} \oplus S\left(G_{1} C^{\prime} \oplus S\left(G_{2} C^{\prime} \oplus \cdots \oplus S G_{k-1} C^{\prime}\right) \ldots\right)}$ is the sign of the new iterated mapping cone structure on $C$, and so $\eta_{F_{*} C^{\prime}}=\eta_{F_{*} C}=\eta_{C}$ as required. 
Theorem 12.14 (Invariance) For a filtered contractible $k$-filtered signed complex $\left(F_{*} C, \eta_{F_{*} C}\right)$ in $\mathrm{A}$

$$
\tau^{\mathrm{NEW}}\left(C, \eta_{C}\right)=i_{*} \tau^{\mathrm{NEW}}\left(\mathbb{G}_{*}(C), \eta_{\mathbb{G}_{*}(C)}\right) \in K_{1}^{\mathrm{iso}}(\mathbb{A}) .
$$

Proof The proof is by induction on $k$. The result is true for $k=0$, since in that case $\left(\mathbb{G}_{*}(C), \eta_{\mathbb{G} *(C)}\right)$ is concentrated in degree 0 , and $\eta_{C}=i_{*} \eta_{\mathbb{G} *(C)}+\eta_{G_{0}(C)}$. Therefore

$$
i_{*} \tau^{\mathrm{NEW}}\left(\mathbb{G}_{*}(C), \eta_{\mathbb{G}_{*}(C)}\right)=\tau^{\mathrm{NEW}}\left(G_{0}(C), \eta_{G_{0}(C)}\right)+i_{*} \eta_{\mathbb{G} *(C)}=\tau^{\mathrm{NEW}}\left(C, \eta_{C}\right) .
$$

So assume that $k \geqslant 1$, and that the result is true for $(k-1)$. Let $\left(F_{*} C^{\prime}, \eta_{F_{*} C^{\prime}}\right)$ be the $(k-1)$-amalgamation of $C$. From the construction of $F_{*} C^{\prime}$, it is clear that

$$
\tau^{\mathrm{NEW}}\left(F_{*} C, \eta_{F_{*} C}\right)=\tau^{\mathrm{NEW}}\left(F_{*} C^{\prime}, \eta_{F_{*} C^{\prime}}\right) \in K_{1}^{\text {iso }}(\mathbb{A}),
$$

and by the inductive hypothesis

$$
\tau^{\mathrm{NEW}}\left(F_{*} C^{\prime}, \eta_{F_{*} C^{\prime}}\right)=i_{*} \tau^{\mathrm{NEW}}\left(\mathbb{G}_{*}\left(C^{\prime}\right), \eta_{\mathbb{G}_{*}}\left(C^{\prime}\right)\right) \in K_{1}^{\mathrm{iso}}(\mathbb{A}) .
$$

By Proposition 12.5 (iv) a filtered contraction $\Gamma: 1 \simeq 0: C \rightarrow C$ of $\left(F_{*} C, \eta_{F_{*} C}\right)$ determines a contraction

$$
\mathbb{G}_{*}(\Gamma): 1 \simeq 0: \mathbb{G}_{*}(C) \rightarrow \mathbb{G}_{*}(C)
$$

of the associated $k$-dimensional chain complex $\mathbb{G}_{*}(C)$ in the signed derived category $\mathbb{S D}(\mathbb{A})$ with

$$
G_{r}(\Gamma)=(-)^{s} \Gamma_{-1}: G_{r}(C)_{s}=C_{r+s, r} \rightarrow G_{r+1}(C)_{s}=C_{r+s+1, r+1} .
$$

In particular, the chain maps

$$
\partial_{k}=d_{*}: G_{k}(C) \rightarrow G_{k-1}(C), \Gamma_{-1}: G_{k-1}(C) \rightarrow G_{k}(C)
$$

are related by a chain homotopy

$$
\Gamma_{0}: \Gamma_{-1} \partial_{k} \simeq 1: G_{k}(C) \rightarrow G_{k}(C) .
$$

Now Proposition 12.7 gives a direct sum system in $\mathbb{S} \mathbb{D}(\mathbb{A})$

$$
G_{k}(C) \stackrel{\partial_{k}}{\underset{\Gamma_{-1}}{\rightleftarrows}} G_{k-1}(C) \underset{\Delta}{\stackrel{g}{\rightleftarrows}} C\left(\partial_{k}\right) .
$$

This defines a $k$-splitting of $\mathbb{G}_{*}(C)$, and by Lemma 12.13 the associated amalgamated complex $\mathbb{G}_{*}\left(C^{\prime}\right)$ is the corresponding abelian folding. By Proposition 12.9 there is defined an isomorphism of chain complexes in $S \mathbb{D}(\mathbb{A})$

$$
\left(\begin{array}{l}
i \\
j
\end{array}\right): \mathbb{G}_{*}(C) \cong \mathbb{G}_{*}\left(C^{\prime}\right) \oplus \mathbb{E}
$$


where $\mathbb{E}$ is the elementary complex in $\mathbb{S} \mathbb{D}(\mathbb{A})$ given by $G_{k}(C) \stackrel{1}{\rightarrow} G_{k}(C)$ in degrees $(k, k-1)$. Since $\eta_{\mathbb{G}_{*}(C)}=\eta_{\mathbb{G}_{*}\left(C^{\prime}\right) \oplus \mathbb{E}}$, the formula in Proposition 12.9 for $\tau^{\text {iso }}\left(\begin{array}{l}i \\ j\end{array}\right)$ gives

$$
i_{*} \tau^{\mathrm{NEW}}\left(\begin{array}{l}
i \\
j
\end{array}\right)=(-)^{k-1} \tau^{\mathrm{NEW}}\left(\left(\begin{array}{c}
g \\
\Gamma_{-1}
\end{array}\right): G_{k-1}(C) \rightarrow \mathcal{C}\left(\partial_{k}\right) \oplus G_{k}(C)\right) \in K_{1}^{\mathrm{iso}}(\operatorname{SDD}(\mathbb{A})) .
$$

By Corollary 12.8 this torsion has image

$$
i_{*} \tau^{\mathrm{NEW}}\left(\begin{array}{l}
i \\
j
\end{array}\right)=0 \in K_{1}^{\mathrm{iso}}(\mathbb{A}),
$$

so that

$$
i_{*} \tau^{\mathrm{NEW}}\left(\mathbb{G}_{*}(C)\right)=i_{*} \tau^{\mathrm{NEW}}\left(\mathbb{G}_{*}\left(C^{\prime}\right) \oplus \mathbb{E}\right)=\tau^{\mathrm{NEW}}\left(\mathbb{G}_{*}\left(C^{\prime}\right)\right) \in K_{1}^{\text {iso }}(\mathbb{A})
$$

which gives the inductive step

$$
\begin{aligned}
\tau^{\mathrm{NEW}}\left(F_{*} C, \eta_{F_{*} C}\right) & =\tau^{\mathrm{NEW}}\left(F_{*} C^{\prime}, \eta_{F_{*} C^{\prime}}\right) \\
& =i_{*} \tau^{\mathrm{NEW}}\left(\mathbb{G}_{*}\left(C^{\prime}\right), \eta_{\mathbb{G}_{*}\left(C^{\prime}\right)}\right) \\
& =i_{*} \tau^{\mathrm{NEW}}\left(\mathbb{G}_{*}(C), \eta_{\mathbb{G}_{*}}(C) \in K_{1}^{\mathrm{iso}}(\mathbb{A}) .\right.
\end{aligned}
$$

This completes the proof.

\subsection{The Invariance Theorem for filtered chain equivalences}

We have already defined the filtered mapping cone $F_{*} \mathcal{C}^{\text {fil }}(f)$ of a filtered chain map (see Definition 12.1 (vii)), with associated complex $\mathbb{G}_{*}\left(\mathcal{C}^{\text {fil }}(f)\right)$ given by

$$
G_{r}\left(\mathcal{C}^{\mathrm{fil}}(f)\right)_{s}=G_{r}(D)_{s} \oplus G_{r-1}(C)_{s},
$$

but now we need the signed version.

Definition 12.15 The filtered signed mapping cone $\left(F_{*} \mathcal{C}^{\text {fil }}(f), \eta_{F_{*} \mathcal{C}^{\text {fil }}(f)}\right)$ of a filtered chain map is the $(k+1)$-filtered signed complex defined by the filtered mapping cone $F_{*} \mathcal{C}^{\text {fil }}(f)$ with sign terms

$$
\begin{aligned}
& \eta_{\mathbb{G}_{*}\left(\mathcal{C}^{\mathrm{fil}}(f)\right)}=\eta_{\mathbb{G}_{*}(D) \oplus S \mathbb{G}_{*}(C)} \\
& =-\beta\left(\mathbb{G}_{*}(D), S \mathbb{G}_{*}(C)\right)+\epsilon\left(\mathbb{G}_{*}(D)_{\text {odd }}, \chi\left(S \mathbb{G}_{*}(C)\right)+\eta_{\mathbb{G}_{*}(D)}-\eta_{\mathbb{G}_{*}}(C)\right. \\
& \in \operatorname{im}\left(\epsilon: K_{0}(\mathbb{S} \mathbb{D}(\mathbb{A})) \otimes K_{0}(\mathbb{S} \mathbb{D}(\mathbb{A})) \rightarrow K_{1}^{\text {iso }}(\mathbb{S} \mathbb{D}(\mathbb{A}))\right), \\
& \eta_{G_{r}\left(\mathcal{C}^{\mathrm{fl}}(f)\right)}=\eta_{G_{r}(D) \oplus G_{r-1}(C)} \\
& \epsilon \operatorname{im}\left(\epsilon: K_{0}(\mathbb{A}) \otimes K_{0}(\mathbb{A}) \rightarrow K_{1}^{\text {iso }}(\mathbb{A})\right)(0 \leqslant r \leqslant k+1) .
\end{aligned}
$$

With these sign conventions, the rearrangement map $\rho: \mathcal{C}^{\mathrm{fil}}(f) \rightarrow \mathcal{C}(f)$ is a simple isomorphism. 
Lemma 12.16 $\tau^{\mathrm{NEW}}\left(\left(\mathcal{C}^{\mathrm{fil}}(f), \eta_{\mathcal{C}^{\mathrm{fll}}(f)}\right) \stackrel{\rho}{\rightarrow}\left(\mathcal{C}(f), \eta_{\mathcal{C}(f)}\right)\right)=0 \in K_{1}^{\text {iso }}(\mathbb{A})$.

Proof The proof is by induction on $k$. The result is true for $k=0$, since in that case $F_{*} \mathcal{C}^{\text {fil }}(f)=\mathcal{C}(f)$, with $\mathcal{C}^{\text {fil }}(f)_{r, 0}=D_{r}$ and $\mathcal{C}^{\text {fil }}(f)_{r, 1}=C_{r-1}$, and $\eta_{F_{*} \mathcal{C}^{\text {fil }}(f)}=\eta_{\mathcal{C}(f)}$.

Assume that $k \geqslant 1$, and that the result is true for $(k-1)$. Let

$$
f^{\prime}:\left(F_{*} C^{\prime}, \eta_{F_{*} C^{\prime}}\right) \rightarrow\left(F_{*} D^{\prime}, \eta_{F_{*} D^{\prime}}\right)
$$

be the chain equivalence of the $(k-1)$-amalgamations induced by $f$, with

$$
\tau^{\mathrm{NEW}}\left(\left(\mathcal{C}^{\mathrm{fil}}\left(f^{\prime}\right), \eta_{\mathcal{C}^{\mathrm{fil}}}\left(f^{\prime}\right)\right) \rightarrow\left(\mathcal{C}(f), \eta_{\mathcal{C}(f)}\right)\right)=0 \in K_{1}^{\mathrm{iso}}(\mathbb{A})
$$

by the inductive hypothesis. By Proposition 12.11 the unfiltered complex $\mathcal{C}(f)=$ $\mathcal{C}\left(f^{\prime}\right)$ has two iterated mapping cone descriptions: from the signed filtered complexes $F_{*} E=\mathcal{C}^{\text {fil }}(f)$ and $F_{*} E^{\prime}=\mathcal{C}^{\text {fil }}\left(f^{\prime}\right)$ respectively, we have signed complexes $T_{k, 0}(E)$ and $T_{k, 0}\left(E^{\prime}\right)$, and a commutative diagram

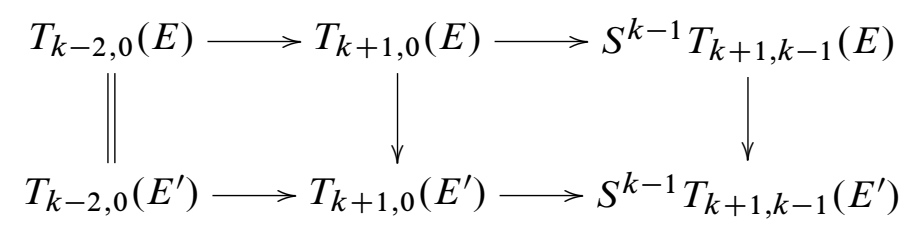

But $\eta_{E}=\eta_{E^{\prime}}$, so the rearrangement map $\rho: E \rightarrow E^{\prime}$ has same absolute torsion as the following composite (distribute $S$, then interchange)

$$
\begin{gathered}
S^{k-1}\left(G_{k-2}(C) \oplus S\left(G_{k}(D) \oplus G_{k-1}(C) \oplus S G_{k}(C)\right)\right) \\
\downarrow 1 \oplus S \\
S^{k-1}\left(G_{k-2}(C) \oplus S G_{k}(D) \oplus S\left(G_{k-1}(C) \oplus S G_{k}(C)\right)\right) \\
\downarrow \begin{array}{lll}
\text { flip } \oplus 1 \\
S^{k-1}\left(S G_{k}(D) \oplus G_{k-2}(C) \oplus S\left(G_{k-1}(C) \oplus S G_{k}(C)\right)\right)
\end{array}
\end{gathered}
$$

since $T_{k-2,0}(E)=T_{k-2}\left(E^{\prime}\right)$. Therefore

$$
\tau^{\mathrm{NEW}}\left(E \rightarrow E^{\prime}\right)=(-)^{k-1} i_{*} \epsilon\left(G_{k}(D), G_{k}(C) \oplus S G_{k-1}(C) \oplus G_{k-2}(C)\right) \in K_{1}^{\text {iso }}(\mathbb{A})
$$

from the formulas in [11, Lemma 7]. By Proposition 12.11,

$$
\tau^{\mathrm{NEW}}\left(\mathcal{C}^{\mathrm{fil}}(f) \rightarrow \mathcal{C}\left(f^{\prime}\right)\right)=\tau^{\mathrm{NEW}}\left(E \rightarrow E^{\prime}\right)+i_{*} \eta_{\mathbb{G}_{*}\left(E^{\prime}\right)}-i_{*} \eta_{\mathbb{G}_{*}}(E)
$$


where the sign term from the filtered mapping cones is

$$
\begin{aligned}
\eta_{\mathbb{G}_{*}(E)}-\eta_{\mathbb{G}_{*}\left(E^{\prime}\right)=} & -\beta\left(\mathbb{G}_{*}(D), S \mathbb{G}_{*}(C)\right)+\epsilon\left(\mathbb{G}_{\text {odd }}(D), \chi\left(S \mathbb{G}_{*}(C)\right)\right) \\
& +\beta\left(\mathbb{G}_{*}\left(D^{\prime}\right), S \mathbb{G}_{*}\left(C^{\prime}\right)\right)-\epsilon\left(\mathbb{G}_{\text {odd }}\left(D^{\prime}\right), \chi\left(S \mathbb{G}_{*}\left(C^{\prime}\right)\right)\right)
\end{aligned}
$$

We first notice that $\mathbb{G}_{\text {odd }}(D)-\mathbb{G}_{\text {odd }}\left(D^{\prime}\right)=G_{k}(D)$ and

$$
i_{*} \chi\left(S \mathbb{G}_{*}(C)\right)=i_{*} \chi\left(S \mathbb{G}_{*}\left(C^{\prime}\right)\right)
$$

since $G_{k-1}\left(C^{\prime}\right)=G_{k-1}(C) \oplus S G_{k}(C)$. Therefore

$$
\begin{aligned}
& i_{*} \epsilon\left(\mathbb{G}_{\text {odd }}(D), \chi\left(S \mathbb{G}_{*}(C)\right)\right)-i_{*} \epsilon\left(\mathbb{G}_{\text {odd }}\left(D^{\prime}\right), \chi\left(S \mathbb{G}_{*}\left(C^{\prime}\right)\right)\right) \\
& \quad=i_{*} \epsilon\left(G_{k}(D), \chi\left(S \mathbb{G}_{*}(C)\right)\right)=-i_{*} \epsilon\left(G_{k}(D), \chi\left(\mathbb{G}_{*}(C)\right)\right) .
\end{aligned}
$$

For the $\beta$-terms, we observe that $\beta\left(\mathbb{G}_{*}(D), S \mathbb{G}_{*}(C)\right)=\beta\left(\mathbb{G}_{*}(D), S \mathbb{G}_{*}\left(C^{\prime}\right)\right)$, and compute

$$
\begin{aligned}
i_{*} & \beta\left(\mathbb{G}_{*}(D), S \mathbb{G}_{*}\left(C^{\prime}\right)\right)-i_{*} \beta\left(\mathbb{G}_{*}\left(D^{\prime}\right), S \mathbb{G}_{*}\left(C^{\prime}\right)\right) \\
& =(-)^{k} i_{*}\left[\epsilon\left(G_{k}(D), \sum_{j \geq 1} G_{k-2 j-1}(C)\right)-\epsilon\left(G_{k}(D), \sum_{j \geq 2} G_{k-2 j}(C)\right)\right] .
\end{aligned}
$$

It follows that

$$
i_{*} \eta_{\mathbb{G}_{*}(E)}-i_{*} \eta_{\mathbb{G}_{*}\left(E^{\prime}\right)}=(-)^{k-1} i_{*} \epsilon\left(G_{k}(D), G_{k}(C) \oplus S G_{k-1}(C) \oplus G_{k-2}(C)\right)
$$

so the sign terms cancel and $\tau^{\mathrm{NEW}}\left(\mathcal{C}^{\text {fil }}(f) \rightarrow \mathcal{C}\left(f^{\prime}\right)\right)=0$ as required.

We proceed now to the statement of the Invariance Theorem. It is immediate from the definitions that the associated complex of the filtered mapping cone $\left(F_{*} \mathcal{C}^{\text {fil }}(f), \eta_{F_{*}} \mathcal{C}^{\text {fil }}(f)\right)$ of a chain map $f:\left(F_{*} C, \eta_{F_{*} C}\right) \rightarrow\left(F_{*} D, \eta_{F_{*} D}\right)$ of $k$-filtered signed complexes in $\mathbb{A}$ is the mapping cone of the associated chain map $\mathbb{G}_{*}(f):\left(\mathbb{G}_{*}(C), \eta_{\mathbb{G}_{*}(C)}\right) \rightarrow$ $\left(\mathbb{G}_{*}(D), \eta_{\mathbb{G}_{*}(D)}\right)$ in the signed derived category $\mathbb{S} \mathbb{D}(\mathbb{A})$, so we have

$$
\left(\mathbb{G}_{*}\left(\mathcal{C}^{\mathrm{fil}}(f)\right), \eta_{\mathbb{G}_{*}}\left(\mathcal{C}^{\mathrm{fl}}(f)\right)\right)=\left(\mathcal{C}\left(\mathbb{G}_{*}(f)\right), \eta_{\mathbb{G}_{*}}(D) \oplus S \mathbb{G}_{*}(C)\right) .
$$

By Theorem 12.6, the filtered chain map $f$ is a filtered chain equivalence if and only if $\mathbb{G}_{*}(f)$ is a chain equivalence, or $\mathcal{C}\left(\mathbb{G}_{*}(f)\right)$ is contractible.

Theorem 12.17 (Invariance) The torsion of a filtered chain equivalence

$$
f:\left(F_{*} C, \eta_{F_{*} C}\right) \rightarrow\left(F_{*} D, \eta_{F_{*} D}\right)
$$

of $k$-filtered signed complexes is

$$
\tau^{\mathrm{NEW}}(f)=i_{*} \tau^{\mathrm{NEW}}\left(\mathbb{G}_{*}(f)\right) \in K_{1}^{\mathrm{iso}}(\mathbb{A}) .
$$


Proof By definition

$$
\tau^{\mathrm{NEW}}\left(\mathbb{G}_{*}(f)\right)=\tau^{\mathrm{NEW}}\left(\mathcal{C}\left(\mathbb{G}_{*}(f)\right)\right) \in K_{1}^{\mathrm{iso}}(\mathbb{S} \mathbb{D}(\mathbb{A}))
$$

so we have the formulas

$$
\begin{aligned}
i_{*} \tau^{\mathrm{NEW}}\left(\mathbb{G}_{*}(f)\right)=i_{*} \tau^{\mathrm{NEW}}\left(\mathcal{C}\left(\mathbb{G}_{*}(f)\right)\right) & =i_{*} \tau^{\mathrm{NEW}}\left(\mathbb{G}_{*}\left(\mathcal{C}^{\mathrm{fil}}(f)\right)\right) \\
=\tau^{\mathrm{NEW}}\left(\mathcal{C}^{\mathrm{fil}}(f), \eta_{\mathcal{C}^{\mathrm{fil}}(f)}\right) & =\tau^{\mathrm{NEW}}\left(\mathcal{C}(f), \eta_{\mathcal{C}(f)}\right)=\tau^{\mathrm{NEW}}(f) \in K_{1}^{\mathrm{iso}}(\mathbb{A}) .
\end{aligned}
$$

This completes the theorem.

\subsection{The filtered dual complex}

Let $(\mathbb{A}, *)$ be an additive category with involution (see [26]). We now define an involution on a sub-category of the additive category $\mathbb{S} \mathbb{D}(\mathbb{A})$ (following $[11$, Section $3])$.

Definition 12.18 Given a signed chain complex $C$ in $\mathbb{A}$, the $n$-dual signed chain complex is $\left(C^{n-*}, \eta_{C^{n-*}}\right)$, where

and the sign

$$
d_{C^{n-*}}=(-1)^{r} d_{C}^{*}: C^{n-r}=\left(C_{n-r}\right)^{*} \rightarrow C^{n-r+1}
$$

$$
\eta_{C^{n-*}}=(-)^{n}\left(\eta_{C}\right)^{*}+\beta(C, C)+\alpha_{n}(C) \in K_{1}^{\mathrm{iso}}(\mathbb{A})
$$

where

$$
\alpha_{n}(C)=\sum_{r \equiv n+2, n+3(\bmod 4)} \epsilon\left(C^{r}, C^{r}\right) \in K_{1}^{\text {iso }}(\mathbb{A}) .
$$

For any integer $n \geq 0$, let $\mathbb{S} \mathbb{P} \mathbb{D}_{n}(\mathbb{A})$ denote the full sub-category of $\mathbb{S} \mathbb{D}(\mathbb{A})$ whose objects are signed chain complexes $\left(C, \eta_{C}\right)$ in $\mathbb{A}$ with $\operatorname{dim} C=n$, such that $C^{n-*}$ is chain equivalent to $C$ and $\chi(C)=0$ for $n$ odd.

Remark 12.19 The condition that $\chi(C)=0$ when $n$ is odd is necessary to ensure that $C \mapsto C^{n-*}$ is an additive functor on $\mathbb{S P D}_{n}(\mathbb{A})$. This condition and the requirement that $C^{n-*}$ be chain equivalent to $C$ are both satisfied (by duality) for symmetric Poincaré $n$-complexes of $R$-modules, and these are the main objects of interest in the rest of the paper.

Example 12.20 Let $\mathbb{A}=\mathbb{A}(R)$ denote the additive category of finitely generated based free modules over a ring $R$ with involution. Then $\mathbb{A}(R)$ has an involution $*: \mathbb{A}(R) \rightarrow \mathbb{A}(R)$ (see [26]), and we get an involution on $\mathbb{S P} \mathbb{D}_{n}(R):=\mathbb{S P} \mathbb{D}_{n}(\mathbb{A}(R)$ ) given by

$$
*: C \mapsto C^{n-*} \quad *:(f: C \rightarrow D) \mapsto\left(f^{n-*}: D^{n-*} \rightarrow C^{n-*}\right)
$$

(see [11]). 
We will now define the filtered dual $F_{*}^{\text {dual }} C$ of a $k$-filtered complex $F_{*} C$ (under some filtration assumptions to ensure that each $G_{r}(C)$ is an $n$-dimensional chain complex). It will turn out that the associated complex $\mathbb{G}_{*}\left(F^{\text {dual }} C\right)$ is the $k$-dual of $\mathbb{G}_{*}(C)$. Our main example is the $k$-filtered chain complex $F_{*} C(\widetilde{E})$ of the total space of a fibre bundle $F \rightarrow E \rightarrow B$, where $\operatorname{dim} B=k$ and $\operatorname{dim} F=n$ (see Section 5).

Definition 12.21 Let $F_{*} C$ be a $k$-filtered $(n+k)$-dimensional chain complex in $\mathbb{A}(R)$.

(i) We say that $F_{*} C$ is $n$-admissible if

(a) $C_{r, s}=0$ unless $0 \leqslant s \leqslant k$ and $0 \leqslant r-s \leqslant n$.

(b) $\left(G_{r} C\right)^{n-*}$ is chain equivalent to $G_{r} C$.

(ii) We define the filtered dual $F_{*}^{\text {dual }} C$ of $F_{*} C$ to be the $(n+k)$-filtered complex with modules $\left(F_{*}^{\text {dual }} C\right)_{r, s}=C_{n+k-r, k-s}^{*}$ for $0 \leqslant s \leqslant k$ and differentials $d^{\text {dual }}:\left(F_{*}^{\text {dual }} C\right)_{r} \rightarrow\left(F_{*}^{\text {dual }} C\right)_{r-1}$ the filtered morphism given by the upper triangular matrix with components

$$
d_{j}^{\text {dual }}=(-)^{r+s+j(n+r)} d_{j}^{*}: C_{n+k-r, k-s}^{*} \rightarrow C_{n+k-r+1, k-s+j}^{*} .
$$

The associated complex of $F_{*}^{\text {dual }} C$ will be denoted $\mathbb{G}_{*}\left(F^{\text {dual }} C\right)$.

(iii) We define the signed filtered dual of $F_{*} C$ as follows: Since $F_{*} C$ is $n$-admissible $\mathbb{G}_{*} C$ may be considered to lie in $\mathbb{S P} \mathbb{D}_{n}(R)$. This is a category with involution so the dual sign $\eta_{\left(\mathbb{G}_{*} C\right)^{k-*}} \in K_{1}^{\text {iso }}\left(\mathbb{S P} \mathbb{D}_{n}(\mathbb{A}(R))\right.$ is defined. We have an obvious functor $\mathbb{S P D}_{n}(R) \rightarrow \operatorname{SD}(R)$ so we may consider $\eta_{\left(\mathbb{G}_{*} C\right)^{k-*}}$ to lie in $K_{1}^{\text {iso }}(\mathbb{S} \mathbb{D}(R))$. We define the signed filtered dual of $F_{*} C$ to be the signed filtered complex whose underlying filtered chain complex is $F_{*}^{\text {dual }} C$ and with signs

$$
\begin{aligned}
& \eta_{\mathbb{G}_{*}\left(F_{\text {dual }} C\right)}=\eta_{\left(\mathbb{G}_{*} C\right)^{k-*}} \in K_{1}^{\text {iso }}(\operatorname{SDD}(R)) \\
& \eta_{G_{r}\left(F^{\text {dual }} C\right)}=\eta_{G_{k-r}}(C)^{n-*} \in K_{1}^{\text {iso }}(R) .
\end{aligned}
$$

Remark 12.22 It follows from the condition $\left(G_{r} C\right)^{n-*}$ is chain equivalent to $G_{r} C$ that $\chi\left(F_{*} C\right)$ is zero if $n$ is odd.

Lemma 12.23 The associated complex $\mathbb{G}_{*}\left(F^{\text {dual }} C\right)$ of the filtered dual of $F_{*} C$ is the $k$-dual of $\mathbb{G}_{*}(C)$ in $\mathbb{S P \mathbb { D }}(\mathbb{A})$.

Proof We have $G_{r}\left(F^{\text {dual }} C\right)_{s}=\left(F_{*}^{\text {dual }} C\right)_{r+s, r}=C_{n+k-r-s, k-r}^{*}$ by definition. The internal differential $d_{0}: G_{r}\left(F^{\text {dual }} C\right)_{s} \rightarrow G_{r}\left(F^{\text {dual }} C\right)_{s-1}$ on this term is given by $(-)^{s} d_{0}^{*}$. On the other hand, $G_{k-r}(C)^{n-s}=C_{k-r+n-s, k-r}^{*}$, so the dual complex has the same chain groups as the associated complex. The differential on $G_{k-r}(C)^{n-s}$ is 
again $(-)^{s} d_{0}^{*}$, since $\operatorname{dim} G_{k-r}(C)=n$. The signs on $\mathbb{G}_{*}\left(F^{\text {dual }} C\right)$ were defined to agree with those of the $k$-dual of $\mathbb{G}_{*}(C)$, and in particular $G_{r}\left(F^{\text {dual }} C\right)=\left(G_{k-r} C\right)^{n-*}$ as signed chain complexes, for $0 \leqslant r \leqslant k$.

We define the map

$$
\theta_{F_{*} C}: C^{n+k-*} \rightarrow F_{*}^{\text {dual }} C
$$

as the direct sum of the following sign maps on the $(r, s)$-summands of the underlying modules:

$$
(-)^{s(n+r+1)}: C_{n+k-r, k-s}^{*} \rightarrow C_{n+k-r, k-s}^{*} .
$$

Lemma 12.24 $\theta_{F_{*} C}$ is a chain equivalence of (unfiltered) complexes.

Proof We have a commutative diagram

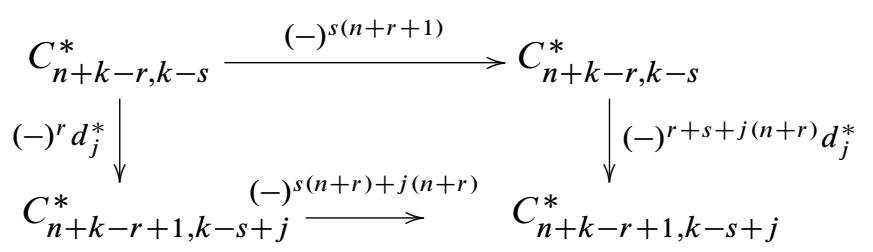

where the vertical maps are the components of the differential on $C^{n+k-*}$ and $F_{*}^{\text {dual }} C$ respectively.

Proposition 12.25 Let $F_{*} C$ be an admissible, signed, $k$-filtered chain complex over $\mathbb{A}(R)$, with $\operatorname{dim} C=n+k$. Then $\tau^{\mathrm{NEW}}\left(\theta_{F_{*}}: C^{n+k-*} \rightarrow F_{*}^{\text {dual }} C\right)=0$.

Proof The proof will be by induction on $m(C)$, defined as the smallest integer $m$ such that $F_{m} C=C$. Suppose first that $m(C)=0$. Then $C_{r}=C_{r, 0}$ and $G_{r}(C)=0$ unless $r=0$. We still allow non-zero signs $\eta_{G_{r}(C)} \in K_{1}(\mathbb{Z})$ if $r>0$. The map $\theta_{F_{*} C}: C^{n+k-r} \rightarrow C^{n+k-r}$ has absolute torsion

$$
\begin{aligned}
\tau^{\mathrm{NEW}}(\theta) & =\sum_{r=0}^{n+k} \tau^{\text {iso }}\left((-)^{k(n+r+1)}\right)+\eta_{F_{*}^{\text {dual }}} C-\eta_{C^{n+k-*}} \\
& =k \cdot \chi\left(C_{\text {odd }}\right)+\frac{1}{2} k(k+1) \chi(C)+\alpha_{n+k}(C)+\alpha_{n}(C) \\
& =\left\{\begin{array}{l}
0 \\
\chi\left(C_{\text {odd }}\right) \\
0 \\
\chi\left(C_{\text {odd }}\right)
\end{array}+\left\{\begin{array}{l}
0 \\
\chi(C) \\
\chi(C) \\
0
\end{array}+\left\{\begin{array} { l } 
{ 0 } \\
{ \chi ( C _ { \text { even } } ) } \\
{ \chi ( C ) } \\
{ \chi ( C _ { \text { odd } } ) }
\end{array} \text { for } k \equiv \left\{\begin{array}{ll}
0 & \\
1 & \bmod 4 \\
2 & \\
3 &
\end{array}\right.\right.\right.\right. \\
& =0 \in K_{1}(\mathbb{Z}) .
\end{aligned}
$$


We now move on to the inductive step. Let $m=M(C)>0$ for $F_{*} C$ and let $F_{*} C^{\prime}$ be the $m$-amalgamation of $F_{*} C$, considered as a $k$-filtered complex, with

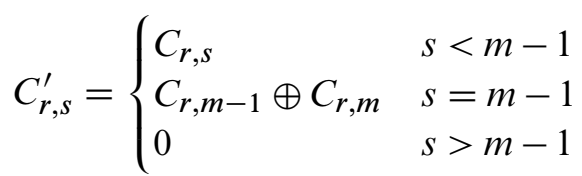

with signs $\eta_{\mathbb{G}_{*}\left(C^{\prime}\right)}=\eta_{\mathbb{G}_{*}(C)}$ and

$$
\eta_{G_{r}\left(C^{\prime}\right)}= \begin{cases}\eta_{G_{r}(C)} & r \neq m-1 \\ \eta_{G_{m-1}(C) \oplus S G_{m}(C)} & r=m-1 .\end{cases}
$$

Note that $M\left(C^{\prime}\right)=m-1$, and that $C=C^{\prime}$ as unfiltered signed chain complexes. Then we have a commutative diagram

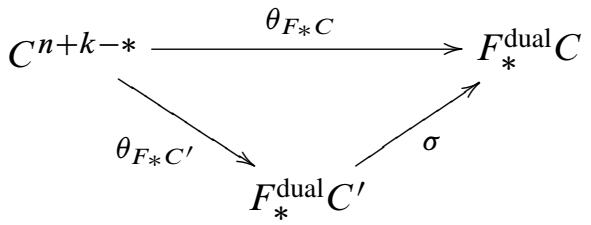

with the map $\sigma: F_{*}^{\text {dual }} C^{\prime} \rightarrow F_{*}^{\text {dual }} C$ induced by the maps on the underlying modules: $\sigma=(-)^{k+1+r}: C_{r, m}^{*} \rightarrow C_{r}^{*}$, and otherwise $\sigma=1$. By our inductive assumption, $\tau^{\mathrm{NEW}}\left(\theta_{F_{*} C^{\prime}}\right)=0$, so it remains to compute $\tau^{\mathrm{NEW}}(\sigma)$.

We use the $k$-filtered complexes $F_{C}$ and $F_{*} C^{\prime}$ to express $C$ and $C^{\prime}$ as an iterated mapping cones. Then

$$
\tau^{\mathrm{NEW}}(\sigma)=\tau^{\text {iso }}(\sigma)+i_{*} \eta_{\mathbb{G}\left(F^{\text {dual }} C\right)}-i_{*} \eta_{\mathbb{G}\left(F^{\text {dual }} C^{\prime}\right)} \in K_{1}(\mathbb{Z})=\mathbb{Z} / 2
$$

where $\tau^{\text {iso }}(\sigma)$ is the sum of the term

$$
\tau^{\mathrm{NEW}}\left((-)^{k+m+n+s+1}: G_{m}(C)^{n-s} \rightarrow S\left(S G_{m}(C)^{n-s}\right)\right)=(1+k+m+n) \cdot \chi\left(G_{m}(C)\right)
$$

by [11, Lemma 18], plus the torsions of rearrangements of the direct sum

$$
S^{k-m}\left(G_{m}(C)^{n-*} \oplus S\left(G_{m-1}(C)^{n-*} \oplus S\left(G_{m-2}(C)^{n-*} \oplus \cdots\right) \cdots\right)\right) .
$$

Note that the chain groups $G_{m}(C)^{n-s}=S\left(S G_{m}(C)^{n-s}\right)$, although these chain complexes have different signs on their differentials. Let $X=S G_{m}(C), Y=G_{m-1}(C)$ and $\left.Z=S\left(G_{m-2}(C)^{n-*} \oplus \cdots\right) \cdots\right)$. Then we rearrange by

$$
\begin{aligned}
S X^{n-*} \oplus S\left(Y^{n-*} \oplus Z\right) \stackrel{a}{\rightarrow} S\left(X^{n-*} \oplus Y^{n-*} \oplus Z\right) & \stackrel{b}{\rightarrow} S\left((X \oplus Y)^{n-*} \oplus Z\right) \stackrel{c}{\rightarrow} S\left((Y \oplus X)^{n-*} \oplus Z\right) .
\end{aligned}
$$


The formulas of [11, Lemma 7] give

$$
\tau^{\mathrm{NEW}}(a)+\tau^{\mathrm{NEW}}(b)+\tau^{\mathrm{NEW}}(c)=\epsilon\left(G_{m}(C), G_{m-2}(C) \oplus \cdots \oplus G_{0}(C)\right) .
$$

Now the sign term $i_{*} \eta_{\mathbb{G}\left(F^{\text {dual }} C\right)}-i_{*} \eta_{\mathbb{G}\left(F^{\text {dual }} C^{\prime}\right)}$ is the sum of two terms

and

$$
\alpha_{k}\left(\mathbb{G}_{*}(C)\right)-\alpha_{k}\left(\mathbb{G}_{*}\left(C^{\prime}\right)\right)=(1+k+m+n) \cdot \chi\left(G_{m}(C)\right)
$$

$$
\beta\left(\mathbb{G}_{*}(C), \mathbb{G}_{*}(C)\right)-\beta\left(\mathbb{G}_{*}\left(C^{\prime}\right), \mathbb{G}_{*}\left(C^{\prime}\right)\right)=\epsilon\left(G_{m}(C), G_{m-2}(C) \oplus \cdots \oplus G_{0}(C)\right)
$$

so $\tau^{\mathrm{NEW}}(\sigma)=0$. Hence $\tau^{\mathrm{NEW}}\left(\theta_{F_{*} C}\right)=\tau^{\mathrm{NEW}}\left(\theta_{F_{*} C^{\prime}}\right)=0$ as required.

Example 12.26 (Tensor products) The following special case is used in deriving the product formula for the absolute torsion of symmetric Poincare spaces (see Proposition $6.5)$.

Lemma 12.27 Let $C$ and $D$ be chain complexes of dimension over $\mathbb{A}(R)$ and $\mathbb{A}(S)$ respectively, where $R$ and $S$ are rings with involution. Let $\operatorname{dim} C=k$ and $\operatorname{dim} D=n$. Then we have a chain equivalence

given by

$$
\theta_{C \otimes D}:(C \otimes D)^{n+k-*} \rightarrow C^{k-*} \otimes D^{n-*}
$$

$$
\theta_{C \otimes D}=(-)^{(k+n+r)(s+n)}: C^{k-r} \otimes D^{n-s} \rightarrow C^{k-r} \otimes D^{n-s} .
$$

Moreover

$$
\tau^{\mathrm{NEW}}\left(\theta_{C \otimes D}\right)=0 \in K_{1}(R \otimes S) .
$$

Proof The associated complex of $F_{*}\left(C^{k-*} \otimes D^{n-*}\right)$ is given by

$$
\mathbb{G}_{*}\left(C^{k-*} \otimes D^{n-*}\right): \cdots \rightarrow C^{k-r} \otimes D^{n-*} \stackrel{(-)^{r} d_{C}^{*} \otimes 1}{\longrightarrow} C^{k-r+1} \otimes D^{n-*}
$$

thus $\mathbb{G}_{*}\left(C^{k-*} \otimes D^{n-*}\right)=\mathbb{G}_{*}(C \otimes D)^{k-*}$ considered as the $k$-dual of a chain complex in $\mathbb{S P D}_{n}(\mathbb{A}(R \otimes S))$. Hence $F_{*}\left(C^{k-*} \otimes D^{n-*}\right)=F_{*}^{\text {dual }}(C \otimes D)$; moreover

$$
\theta_{C \otimes D}=\theta_{F_{*}(C \otimes D)}:(C \otimes D)^{n+k-*} \rightarrow F_{*}\left(C^{k-*} \otimes D^{n-*}\right)=F_{*}^{\text {dual }}(C \otimes D) .
$$

The result now follows from Proposition 12.25.

\section{References}

[1] D R Anderson, The Whitehead torsion of the total space of a fiber bundle, Topology 11 (1972) 179-194 MR0295348

[2] MF Atiyah, The signature of fibre-bundles, from: "Global Analysis (Papers in Honor of K. Kodaira)", Univ. Tokyo Press, Tokyo (1969) 73-84 MR0254864 
[3] A J Casson, Generalisations and applications of block bundles, from: "The Hauptvermutung book", K-Monogr. Math. 1, Kluwer Acad. Publ., Dordrecht (1996) 33-67 MR1434102

[4] S S Chern, F Hirzebruch, J-P Serre, On the index of a fibered manifold, Proc. Amer. Math. Soc. 8 (1957) 587-596 MR0087943

[5] H Endo, A construction of surface bundles over surfaces with non-zero signature, Osaka J. Math. 35 (1998) 915-930 MR1659565

[6] I Hambleton, A Ranicki, L Taylor, Round L-theory, J. Pure Appl. Algebra 47 (1987) 131-154 MR906966

[7] F Hirzebruch, The signature of ramified coverings, from: "Global Analysis (Papers in Honor of K. Kodaira)", Univ. Tokyo Press, Tokyo (1969) 253-265 MR0258060

[8] F Hirzebruch, W D Neumann, S S Koh, Differentiable manifolds and quadratic forms, Lecture Notes in Pure and Applied Mathematics 4, Marcel Dekker, New York (1971) MR0341499 (Appendix II by W. Scharlau)

[9] S Klaus, P Teichner, Private communication (2003)

[10] K Kodaira, A certain type of irregular algebraic surfaces, J. Analyse Math. 19 (1967) 207-215 MR0216521

[11] A J Korzeniewski, Absolute Whitehead torsion, Geom. Topol. 11 (2007) 215-249

[12] W Lück, The transfer maps induced in the algebraic $K_{0}$ - and $K_{1}$-groups by a fibration. I, Math. Scand. 59 (1986) 93-121 MR873491

[13] W Lück, The transfer maps induced in the algebraic $K_{0}$ - and $K_{1}$-groups by a fibration. II, J. Pure Appl. Algebra 45 (1987) 143-169 MR889589

[14] W Lück, A Ranicki, Surgery obstructions of fibre bundles, J. Pure Appl. Algebra 81 (1992) 139-189 MR1176019

[15] S Maumary, Contributions à la théorie du type simple d'homotopie, Comment. Math. Helv. 44 (1969) 410-437 MR0266210

[16] W Meyer, Die Signatur von Flächenbündeln, Math. Ann. 201 (1973) 239-264 MR0331382

[17] J Milnor, Whitehead torsion, Bull. Amer. Math. Soc. 72 (1966) 358-426 MR0196736

[18] H J Munkholm, Whitehead torsion for PL fiber homotopy equivalences, from: "Algebraic topology, Waterloo, 1978 (Proc. Conf., Univ. Waterloo, Waterloo, Ont., 1978)”, Lecture Notes in Math. 741, Springer, Berlin (1979) 90-101 MR557164

[19] W D Neumann, Multiplicativity of signature, J. Pure Appl. Algebra 13 (1978) 19-31 MR508726

[20] E K Pedersen, Geometrically defined transfers, comparisons, Math. Z. 180 (1982) 535-544 MR667007 
[21] H Putz, Triangulation of fibre bundles, Canad. J. Math. 19 (1967) 499-513 MR0212810

[22] A Ranicki, The algebraic theory of surgery. I. Foundations, Proc. London Math. Soc. (3) 40 (1980) 87-192 MR560997

[23] A Ranicki, The algebraic theory of surgery. II. Applications to topology, Proc. London Math. Soc. (3) 40 (1980) 193-283 MR566491

[24] A Ranicki, The algebraic theory of torsion. I. Foundations, from: "Algebraic and geometric topology (New Brunswick, N.J., 1983)", Lecture Notes in Math. 1126, Springer, Berlin (1985) 199-237 MR802792

[25] A Ranicki, The algebraic theory of torsion. II. Products, K-Theory 1 (1987) 115-170 MR899919

[26] A Ranicki, Additive L-theory, K-Theory 3 (1989) 163-195 MR1029957

[27] H Seifert, W Threlfall, Seifert and Threlfall: a textbook of topology, Pure and Applied Mathematics 89, Academic Press, New York (1980) MR575168

[28] C T C Wall, Surgery on compact manifolds, London Mathematical Society Monographs 1, Academic Press, London (1970) MR0431216

[29] J HC Whitehead, Simple homotopy types, Amer. J. Math. 72 (1950) 1-57 MR0035437

IH: Department of Mathematics \& Statistics, McMaster University

Hamilton, Ontario, L8S 4K1, Canada

AJK, AAR: School of Mathematics, University of Edinburgh

Edinburgh, EH9 3JZ, United Kingdom

ian@math.mcmaster.ca, ajkorzeniewski@yahoo.co.uk, A.Ranicki@ed.ac.uk

Proposed: Tom Goodwillie

Received: 20th April 2005

Seconded: Colin Rourke and Peter Teichner

Accepted: 6th January 2007 